\title{
The first law of general quantum resource theories
}

\author{
Carlo Sparaciari ${ }^{1}$, Lídia del Rio ${ }^{2}$, Carlo Maria Scandolo ${ }^{3}$, Philippe Faist ${ }^{4,5}$, and Jonathan \\ Oppenheim ${ }^{1}$

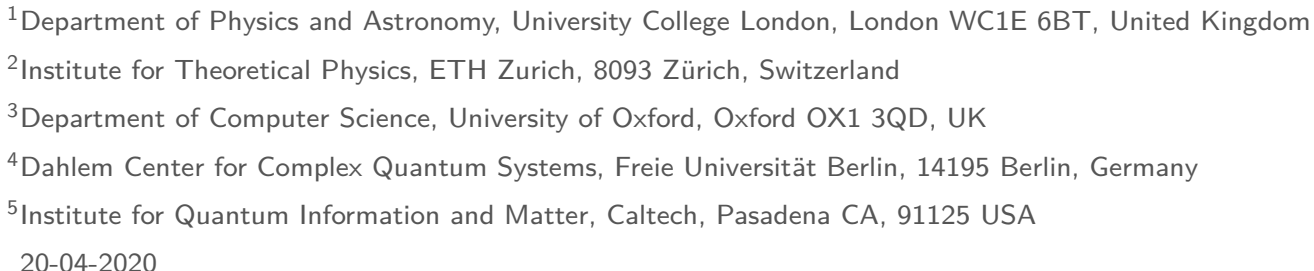

We extend the tools of quantum resource theories to scenarios in which multiple quantities (or resources) are present, and their interplay governs the evolution of physical systems. We derive conditions for the interconversion of these resources, which generalise the first law of thermodynamics. We study reversibility conditions for multi-resource theories, and find that the relative entropy distances from the invariant sets of the theory play a fundamental role in the quantification of the resources. The first law for general multi-resource theories is a single relation which links the change in the properties of the system during a state transformation and the weighted sum of the resources exchanged. In fact, this law can be seen as relating the change in the relative entropy from different sets of states. In contrast to typical single-resource theories, the notion of free states and invariant sets of states become distinct in light of multiple constraints. Additionally, generalisations of the Helmholtz free energy, and of adiabatic and isothermal transformations, emerge. We thus have a set of laws for general quantum resource theories, which generalise the laws of thermodynamics. We first test this approach on thermodynamics with multiple conservation laws, and then apply it to the theory of local operations under energetic restrictions.

\section{Contents}

1 Introduction 2

2 Framework for multi-resource theories 4

3 Reversible multi-resource theories $\quad 8$

4 Bank states, interconversion relations, and the first law 16

5 Examples 24

6 Conclusions 31

A Reversibility and asymptotic equivalence for single-resource theories 34

$\begin{array}{ll}\text { B Convex boundary and bank states } & 37\end{array}$

$\begin{array}{ll}\text { C Energy-entanglement interconversion protocol } & 40\end{array}$

$\begin{array}{lr}\text { D Proofs } & 41\end{array}$

Carlo Sparaciari: c.sparaciari@ucl.ac.uk 


\section{Introduction}

Resource theories. Resource theories are a versatile set of tools developed in quantum information theory. They are used to describe the physical world from the perspective of an agent, whose ability to modify a quantum system is restricted by either practical or fundamental constraints. These limitations mean that while some states can still be created under the restricted class of operations (the free or invariant set of states), other state transformations can only be done with the help of additional resources. The goal of resource theories is then to quantify this cost, and to consequently assign a price to every state of the system, from the most expensive to the free ones. Because of their very general structure, which only involves the set of states describing a quantum system and a given set of allowed operations for acting on such system, resource theories can be used to study many different branches of quantum physics, from entanglement theory [1-5] to thermodynamics [6-13], from asymmetry [14-16] to the theory of magic states [17-19]. Additionally, these theories can often be formulated within more abstract, axiomatic frameworks [20-26].

Thanks to the underlying common structure present in all the theories described within this framework, one can find general results which apply to all. For example, a resource theory may be equipped with a zeroth, second, and even third law, i.e., relations that regulate the different aspects of the theory, which are reminiscent of the Laws of Thermodynamics. In fact, we have that the zeroth law for resource theories states that there exists equivalence classes of free states, and that states from one of these classes are the only ones that can be freely added to the system without trivialising the theory [27]. The second law of resource theories states that some quantities, linked to the amount of resource contained in a system, never increase under the action of the allowed operations [28], and for reversible resource theories satisfying modest assumptions, this quantity is unique [29-33] — an example of this is the free energy, which is a monotone in thermodynamics as it decreases in any cyclic process, and the local entropy for pure state entanglement theory. Finally, one might have a generalisation of the third law which places limitations on the time needed to reach a state when starting from another one, rather than simply telling us whether such transformation is possible or not [34]. With the present work, we aim to derive the first law for resource theories, and to do so we will have to extend the framework so as to include multiple resources. The law we derive connects the amount of different resources exchanged during a state transformation to the change, quantified by a specific monotone, between the initial and final state of the system. When considering thermodynamics, this law connects the amount of work and heat exchanged during a process to the internal energy of the systems.

Multiple resources. It is often the case that many resources are needed to perform a given task. For instance, thermodynamics can be understood as a resource theory with multiple resources [35, 36], where in order to transform the state of the system we need both energy and information, or equivalently, work and heat. As another example, some quantum computational schemes consider the idealized case in which the input qubits are pure, and the gates acting on them create coherence. In order to better understand the role played in quantum computation by these two resources, coherence and purity, a possible approach might consist in combining the resource theories of purity $[7,37]$ and coherence [3840] together. Other examples of theories in which multiple resources are considered can be found in the literature $[35,36,41-47]$. Given the success of resource theories to describe physical situations where only one resource is involved, it seems natural to ask the question whether the framework can be extended to the case in which more resources are involved. For example, it is known that the resource theoretic approach to thermodynamics allows us to derive a second law relation even in the case in which many (commuting, non-commuting) conserved quantities are present [48-52], and one can consider trade-offs of these [53]. We are thus interested in understanding if one can extend these results to other resource theories, and whether a first law of general resource theories exists.

Contribution of this work. In this paper we present a framework for resource theories with multiple resources, introduced in Sec. 2. In our framework we first consider the different constraints and conservation laws that the model needs to satisfy, and for each of these constraints, we introduce the corresponding single-resource theory. Then, we define the class of allowed operations of the multiresource theory as the set of maps lying in the intersection of all the classes of allowed operations of the single-resource theories. Due to this construction, we find that a multi-resource theory with $m$ resources has at least $m$ invariant sets (i.e., sets of states that are mapped into themselves by the action of the allowed operations of the theory), each of them corresponding to the set of free states of one of the $m$ single-resource theories. In order to make the paper self-contained, in Sec. 2 we also provide a brief 

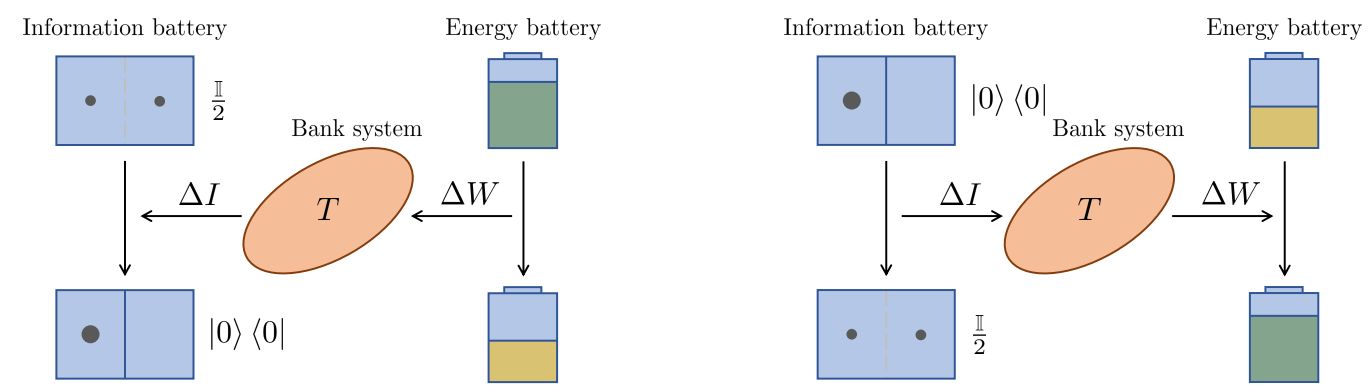

Figure 1: An example of a multi-resource theory is thermodynamics, where energy and information are resources which can be inter-converted. In the figure, we represent three different systems. The main system is a Szilárd box, i.e., a box which can be divided in two partitions, here containing a single particle of gas. We can either know in which side of the box the particle is, or we might not have this information (if, for instance, the partition is removed and the particle is free to move between sides). We additionally have a thermal reservoir surrounding the box, with a well-defined temperature $T$, and we have an ancillary system that we use to store energy (or work), which we refer to as the battery. We can then consider the following two processes. Left. Landauer's erasure is the process of converting some of the energy contained in the battery, $\Delta W$, into information, $\Delta I$, which is then used to reset the state of the particle in the box (from completely unknown, $\frac{\mathbb{I}}{2}$, to perfectly known, $|0\rangle$, in this case). The conversion is realised using the thermal environment, and energy and information are exchanged at the rate $k_{B} T$, which only depends on the properties (the temperature) of the reservoir. Right. In the other direction we can convert information, $\Delta I$, into work, $\Delta W$, at the same exchange rate. Information is extracted form the box, and converted using the thermal bath into energy, which is then stored in the battery. Here, we generalise the function of the thermal reservoir to other multi-resource theories, and we name this system the bank, since it allows for the exchange of one resource into another.

review of the resource theoretic formalism (see Ref. [32, 54] for reviews on this topic).

We then study, in Sec. 3, the properties of general multi-resource theories in the asymptotic limit, that is, when the agent is allowed to act globally over many identical copies of the system. This limit is of fundamental importance in resource theories since it allows us to investigate reversibility and the emergence of unique measures for quantifying different resources [32]. In a reversible theory, we have that the resources consumed to perform a given state transformation can always be completely recovered with the reverse transformation, so that no resource is ever lost. In single-resource theories, we can rephrase this notion of reversibility in terms of rates of conversion, but for general multi-resource theories this is not always possible. As a result, we focus our study on multi-resource theories that satisfy an additional property, which we refer to as the asymptotic equivalence property $[23,35]$, see Def. 1 below. We show that, when a multi-resource theory satisfies the asymptotic equivalence property, there is a unique measure associated with each resource present in the theory. Furthermore, when the invariant sets of the theory satisfy some natural properties, we find that the unique measures are given by the (regularised) relative entropy distances from these sets, each of those associated with a different resource. Finally, we show that when a resource theory satisfies asymptotic equivalence, it is also reversible in the sense that resources are never lost during a state transformation, and they can be recovered. This result can be seen as the extension of what has already been shown for reversible single-resource theories [28, 29, 32, 33].

In Sec. 4 we address the question of whether it is possible to exchange resources. We consider the case in which different resources are individually stored in separate systems, which we call batteries. Then, we investigate under which conditions it is possible to find an additional system, which we refer to as a bank $k^{1}$, that allows us to reduce the amount of resource contained in one battery while simultaneously increasing the amount of resource in another battery. During such conversion, we ask the bank not to change its properties - with respect to a specific measure defined in Eq. (37) - so as to be able to use this system again. For example, in thermodynamics the thermal bath plays the role of the bank, as it allows us to exchange energy for information and vice versa, see Fig. 1. In order to study interconversion, we demand the invariant sets of the theory to satisfy an "additivity" condition,

\footnotetext{
${ }^{1}$ We apologise in advance for introducing this terminology into the field of resource theories, but the banks considered here exchange resources without charging interest or fees, and are thus more akin to community cooperative banks than their more exploitative cousins.
} 
which is satisfied by some resource theories, for example by thermodynamics and purity theory. We find that a multi-resource theory needs to have an empty set of free states for a bank to exist, and when this condition is satisfied we derive an interconversion relation, see Thm. 9, which defines the rates at which resources are exchanged.

We additionally show that, when the agent is allowed to use batteries and bank, they can perform any state transformation using variable amounts of resources. Indeed, since the agent can use the bank to inter-convert between resources, they can decide to invest a higher amount of one resource to save on the others. This freedom is reflected in our framework by a single relation, the first law of resource theories, which connects the different resources, each of them weighted by the corresponding exchange rate, to the change of a particular monotone between the initial and final state of the system, see Cor. 13. This equality is a generalisation of the first law of thermodynamics, where the sum of the work performed on the system and the heat absorbed from the environment is equal to the change in internal energy of the system. In fact, the first law of thermodynamics can be understood as equating various relative entropy distances which quantify different types of resources, as we discuss at the beginning of Sec. 4.

Finally, in Sec. 5 we provide two examples of multi-resource theories which admit an interconversion relation between their resources. The first example concerns thermodynamics of multiple conserved quantities, for which the interconversion of resources was shown in Ref. [48]. The second example concerns the theory of local control under energy restrictions. Here we consider a system with a nonlocal Hamiltonian, and we assume that the experimentalists acting on this system only have access to a portion of the system. In this scenario, the entanglement between the different portions of the system and the overall energy of the global system are the main resources of the theory, and we study under which conditions we can inter-convert energy and entanglement. For a summary of how to apply our work to an arbitrary resource theory, see the flowchart in Fig. 7.

\section{Framework for multi-resource theories}

Let us now introduce the framework for multi-resource theories. A multi-resource theory is useful when we need to describe a physical task or process which is subjected to different constraints and conservation laws. The first step consists in associating each of these constraints with a single-resource theory, whose class of allowed operations satisfies the specific constraint or conservation law. The multi-resource theory is then obtained by defining its class of allowed operations as the intersection between the sets of allowed operations of the different single-resource theories previously defined. In this way, we are sure of acting on the quantum system with operations that do not violate the multiple constraints imposed on the task.

\subsection{Single-resource theory}

For simplicity, we restrict ourselves to the study of finite-dimensional quantum systems. Therefore, the system under investigation is described by a Hilbert space $\mathcal{H}$ with dimension $d$. The statespace of this quantum system is given by the set of density operators acting on the Hilbert space, $\mathcal{S}(\mathcal{H})=\{\rho \in \mathcal{B}(\mathcal{H}) \mid \rho \geq 0, \operatorname{Tr}[\rho]=1\}$, where $\mathcal{B}(\mathcal{H})$ is the set of bounded operators acting on $\mathcal{H}$. A single-resource theory for the quantum system under examination is defined through a class of allowed operations $\mathcal{C}$, that is, a constrained set of completely positive maps acting on the state-space $\mathcal{S}(\mathcal{H})^{2}$ [29]. The constraints posed on the set of allowed operations are specific to the resource theory under consideration. For example, in the theories that study entanglement it is often the case that we constrain the set of allowed operations to be composed by the maps that are local, and only make use of classical communication [1]. In asymmetry theory, instead, we only allow the maps whose action is covariant with respect to the elements of a given group [14]. Furthermore, in the resource theoretic

\footnotetext{
${ }^{2}$ Although the operations we consider are endomorphisms of a given state space, our formalism is still able to describe the general case in which the agent modifies the quantum system. If the agent's action transforms the state of the original system, associated with $\mathcal{H}_{1}$, into the state of a final system $\mathcal{H}_{2}$, we can model this action with a map acting on the state space of $\mathcal{H}=\mathcal{H}_{1} \otimes \mathcal{H}_{2}$. Suppose the operation maps $\rho_{1} \in \mathcal{S}\left(\mathcal{H}_{1}\right)$ into $\sigma_{2} \in \mathcal{S}\left(\mathcal{H}_{2}\right)$. Then, the map acting on $\mathcal{S}(\mathcal{H})$ takes the state $\rho_{1} \otimes \gamma_{2}$ and outputs the state $\gamma_{1}^{\prime} \otimes \sigma_{2}$, where $\gamma_{1}$ and $\gamma_{2}^{\prime}$ are free states for the systems described by $\mathcal{H}_{2}$ and $\mathcal{H}_{1}$, respectively.
} 
approach to thermodynamics we can, without loss of generality, constrain this set to those operations, known as Thermal Operations, which preserve the energy of a closed system, and can thermalise the system with respect to a background temperature $[6,10,11,55]$. Once the set of allowed operations is defined, it is usually possible to identify which states in $\mathcal{S}(\mathcal{H})$ are resourceful, and which ones are not. In particular, the set of free states for a single-resource theory, $\mathcal{F} \subset \mathcal{S}(\mathcal{H})$, is composed of those states that can always be prepared using the allowed operations, no matter the initial state of the system. Mathematically, this set of states is defined as

$$
\mathcal{F}=\{\sigma \in \mathcal{S}(\mathcal{H}) \mid \forall \rho \in \mathcal{S}(\mathcal{H}), \exists \mathcal{E} \in \mathcal{C}: \mathcal{E}(\rho)=\sigma\}
$$

For example, in entanglement theory the free states are the separable states, in asymmetry theory they are the ones that commute with the elements of the considered group, and in thermodynamics they are the thermal states at the background temperature.

An invariant set is a set of states that is preserved under action of any allowed operation. From the definition of free states in Eq. (1), it is easy to show that $\mathcal{F}$ is an invariant set, and we write this as $\mathcal{E}(\mathcal{F}) \subseteq \mathcal{F}$ for all $\mathcal{E} \in \mathcal{C}$. It is worth noting that while the set of free states is invariant, the opposite clearly does not need to be true. In particular, when we study multi-resource theory, we will see that several invariant sets can be found, and still there might be no free set for the theory. Due to the invariant property of free states, we can also define the class of allowed operations in a different way. Instead of considering the specific constraints defining the set of allowed operations $\mathcal{C}$, we can simply assume that this set is a subset of the bigger class of completely positive and trace preserving (CPTP) maps

$$
\tilde{\mathcal{C}}=\{\mathcal{E}: \mathcal{B}(\mathcal{H}) \rightarrow \mathcal{B}(\mathcal{H}) \mid \mathcal{E}(\mathcal{F}) \subseteq \mathcal{F}\}
$$

that is, the set of maps for which the free states $\mathcal{F}$ form an invariant set. It is worth noting that $\mathcal{C}$ is often a proper subset of $\tilde{\mathcal{C}}$. For example, in entanglement theory, we have that $\mathcal{C}$ might be composed by local operations and classical communication (LOCC), which is a proper subset of the set of all quantum channels which preserve the separable states. Indeed, the map that swaps between the local states describing the quantum system is clearly not LOCC, but it preserves separable states [56].

We can also extend the single-resource theory to the case in which we consider $n \in \mathbb{N}$ copies of the quantum system. The class of allowed operations, which in this case we refer to as $\mathcal{C}^{(n)}$, is still defined by the same constraints, but now acts on $\mathcal{S}\left(\mathcal{H}^{\otimes n}\right)$, the state-space of $n$ copies of the system. For example, in the resource theory of thermodynamics with Thermal Operations we have that the energy of a closed system needs to be exactly conserved. For a single system, this implies that the operations need to commute with the Hamiltonian $H^{(1)}$. For $n$ non-interacting copies of the system, instead, the operations commute with the global Hamiltonian $H_{n}=\sum_{i=1}^{n} H_{i}^{(1)}$. Within the state-space $\mathcal{S}\left(\mathcal{H}^{\otimes n}\right)$, we can find the set of free states, $\mathcal{F}^{(n)} \subset \mathcal{S}\left(\mathcal{H}^{\otimes n}\right)$. It is worth noting that the set of free states for $n$ copies of the system is such that $\mathcal{F}^{\otimes n} \subseteq \mathcal{F}^{(n)}$, that is, it contains more states than just the tensor product of $n$ states in $\mathcal{F}$. This is the case, for example, of entanglement theory, where among the free states for two copies of the system we can find states that are locally entangled, since each agent is allowed to entangle the partitions of the system they own. On the contrary, the two sets coincide for any $n \in \mathbb{N}$ for the resource theory of thermodynamics, where the free state is the Gibbs state of a given Hamiltonian. Anyway, it is still the case that $\mathcal{F}^{(n)}$ is invariant under the class $\mathcal{C}^{(n)}$, and therefore we can think of the set of allowed operations acting on $n$ copies of the system as a subset of the bigger set of CPTP maps

$$
\tilde{\mathcal{C}}^{(n)}=\left\{\mathcal{E}_{n}: \mathcal{B}\left(\mathcal{H}^{\otimes n}\right) \rightarrow \mathcal{B}\left(\mathcal{H}^{\otimes n}\right) \mid \mathcal{E}_{n}\left(\mathcal{F}^{(n)}\right) \subseteq \mathcal{F}^{(n)}\right\}
$$

Thus, in order to extend a single-resource theory to the many-copy case, we need to take into account the sequence of all sets of allowed operations $\mathcal{C}^{(n)}$, where $n \in \mathbb{N}$ is the number of copies of the system the maps are acting on.

It is worth noting that the allowed operations we have introduced keep the number of copies of the system fixed, see Eq. (3). Indeed, we only consider these maps because, when the number of input and output systems of a quantum channel changes, the internal structure of the channel involves the discarding (or the addition) of some of these systems. However, in a (reversible) resource theory, one can perform such operations only if the amount of resources is kept constant. This is certainly possible if we are to add or trace out some free states of the theory (which do not contain any resource), but as 
we will see in the next section, multi-resource theory not always have any free states. For this reason, we decide to only focus on maps that conserve the number of systems, even for single-resource theories.

We can now address the problem of quantifying the amount of resource associated with different states of the quantum system. In resource theories, a resource quantifier is called monotone. This object is a function $f$ from the state-space $\mathcal{S}(\mathcal{H})$ to the set of real numbers $\mathbb{R}$, which satisfies the following property,

$$
f(\mathcal{E}(\rho)) \leq f(\rho), \quad \forall \rho \in \mathcal{S}(\mathcal{H}), \forall \mathcal{E} \in \mathcal{C} .
$$

The above inequality can be interpreted as a "second law" for the resource theory, since there is a quantity (the monotone) that never increases as we act on the system with allowed operations. In the thermodynamic case, in fact, we know that the Second Law of Thermodynamics imposes that the entropy of a closed system can never decrease as time goes by. We can extend the definition of monotones to the case in which we consider $n$ copies of the system. In this case, the function $f$ maps states in $\mathcal{S}\left(\mathcal{H}^{\otimes n}\right)$ into $\mathbb{R}$, and an analogous relation to the one of Eq. (4) holds, this time for states in $\mathcal{S}\left(\mathcal{H}^{\otimes n}\right)$ and the set of allowed operations $\mathcal{C}^{(n)}$. Finally, we can also define the regularisation of a monotone $f$ as

$$
f^{\infty}(\rho)=\lim _{n \rightarrow \infty} \frac{f\left(\rho^{\otimes n}\right)}{n},
$$

where $\rho \in \mathcal{S}(\mathcal{H})$, and $\rho^{\otimes n} \in \mathcal{S}\left(\mathcal{H}^{\otimes n}\right)$. Notice that, given a generic monotone $f$, we need the above limit to exist and be finite in order to define its regularisation.

For each resource theory there exists several monotones, and we can always build one out of a contractive distance [33]. Consider the distance $C(\cdot, \cdot): \mathcal{S}(\mathcal{H}) \times \mathcal{S}(\mathcal{H}) \rightarrow \mathbb{R}$ such that

$$
C(\mathcal{E}(\rho), \mathcal{E}(\sigma)) \leq C(\rho, \sigma), \quad \forall \rho, \sigma \in \mathcal{S}(\mathcal{H}), \forall \mathcal{E} \text { CPTP map. }
$$

Then, a monotone for the single-resource theory with allowed operations $\mathcal{C}$ and free states $\mathcal{F}$ is

$$
M_{\mathcal{F}}(\rho)=\inf _{\sigma \in \mathcal{F}} C(\rho, \sigma),
$$

where it is easy to show that $M_{\mathcal{F}}$ satisfies the property of Eq. (4), which follows from the fact that $\mathcal{F}$ is invariant under the set of allowed operations $\mathcal{C}$, and from the contractivity of $C(\cdot, \cdot)$ under any CPTP map. A specific example of a monotone obtained from a contractive distance is the relative entropy distance from the set $\mathcal{F}$. Consider two states $\rho, \sigma \in \mathcal{S}(\mathcal{H})$, such that $\operatorname{supp}(\rho) \subseteq \operatorname{supp}(\sigma)$. Then, we define the relative entropy between these two states as

$$
D(\rho \| \sigma)=\operatorname{Tr}[\rho(\log \rho-\log \sigma)] .
$$

The relative entropy is contractive under CPTP maps [57], and even if it does not satisfy all the axioms to be a metric ${ }^{3}$ over $\mathcal{S}(\mathcal{H})$, we can still obtain a monotone out of this quantity, building it as in Eq. (7). This monotone is

$$
E_{\mathcal{F}}(\rho)=\inf _{\sigma \in \mathcal{F}} D(\rho \| \sigma),
$$

and is known as the relative entropy distance from $\mathcal{F}$. When the separable states form the set $\mathcal{F}$, for example, the monotone is the relative entropy of entanglement [3]. It is worth noting that, in order for $E_{\mathcal{F}}$ to be well-defined, the set $\mathcal{F}$ has to contain at least one full-rank state.

\subsection{Multi-resource theory}

Let us consider the case in which we can identify in the theory a number $m>1$ of resources, which can arise from some conservation laws, or from some constraints. We now introduce a multi-resource theory with these $m$ resources. The quantum system under investigation is the same as in the previous section, described by the states in the state-space $\mathcal{S}(\mathcal{H})$. For the $i$-th resource of interest, where $i=1, \ldots, m$, we consider the corresponding single-resource theory $\mathrm{R}_{i}$, defined by the set of allowed operations $\mathcal{C}_{i}$ acting on the state-space $\mathcal{S}(\mathcal{H})$. We denote the set of free states of this single-resource theory as

\footnotetext{
${ }^{3}$ The relative entropy is non-negative for any two inputs, and zero only when the two inputs coincide, but it is not symmetric, nor does it satisfy the triangular inequality.
} 

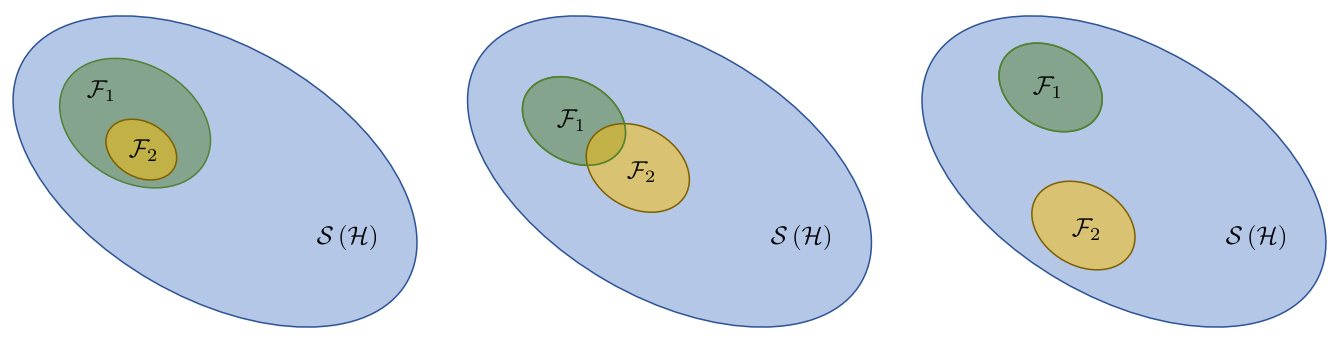

Figure 2: The structure of the sets of free states for two single-resource theories which compose a multi-resource theory. These sets are invariant under the allowed operations of the resulting multi-resource theory. For theories with $m>2$ resources, the structure of the free sets can be obtained by composing the three fundamental scenarios presented here. Left. The invariant set $\mathcal{F}_{2}$ is a subset of $\mathcal{F}_{1}$. This multi-resource theory has a set of free states, which coincides with $\mathcal{F}_{2}$. An example of such a theory is that of coherence [39] and purity [37], where the invariant sets are incoherent states with respect to a given basis and the maximally-mixed state, respectively. Centre. The two invariant sets intersect each other. This theory has a set of free states which coincides with the intersection, $\mathcal{F}_{1} \cap \mathcal{F}_{2}$. An example of multi-resource theory with this structure concerns tripartite entanglement for systems $A, B$, and $C$. The allowed operations of this theory are defined by the intersection of the operations associated with the theories of bipartite entanglement for systems $A B$ and $C$, systems $A C$ and $B$, and systems $A$ and $B C$. Notice that this theory does not coincide with the theory of tripartite LOCC, since some of the free states are entangled [58]. Right. The two invariant sets are separated. Consequently, the theory does not have any free states. In this situation, one can find an interconversion relation between the resources, as shown in Sec. 4.3. An example of a multi-resource theory with this structure is thermodynamics of closed systems. If the agent does not have perfect control on the reversible operations they implement, and the closed system is coupled to a sink of energy (an ancillary system which can only absorb energy), then the allowed operations are given by the intersection between the set of mixtures of unitary operations, and the set of average-energy-non-increasing maps. In this case, the maximally-mixed state and the ground state of the Hamiltonian are the two invariant sets of the theory. Notice that the set of energy-preserving unitary operations, considered in Ref. [35], is a subset of this bigger set.

$\mathcal{F}_{i} \subset \mathcal{S}(\mathcal{H})$, and we recall that any allowed operation in $\mathcal{C}_{i}$ leaves this set invariant. Therefore, we can consider the class of allowed operation as a subset of the set of CPTP maps

$$
\tilde{\mathcal{C}}_{i}=\left\{\mathcal{E}_{i}: \mathcal{B}(\mathcal{H}) \rightarrow \mathcal{B}(\mathcal{H}) \mid \mathcal{E}_{i}\left(\mathcal{F}_{i}\right) \subseteq \mathcal{F}_{i}\right\} .
$$

We can also extend the resource theory $\mathrm{R}_{i}$ to the case in which we consider more than one copy of the system, following the same procedure used in the previous section. Then, the class of allowed operations $\mathcal{C}_{i}^{(n)}$ acting on $n$ copies of the system is a subset of the set of operations which leave $\mathcal{F}_{i}^{(n)} \subset \mathcal{S}\left(\mathcal{H}^{\otimes n}\right)$ invariant, see Eq. (3).

Once all the single-resource theories $\mathrm{R}_{i}$ 's are defined, together with their sets of allowed operations, we can build the multi-resource theory $R_{\text {multi }}$ for the quantum system described by the Hilbert space $\mathcal{H}$. The set of allowed operations for this theory is given by the maps contained in the intersection ${ }^{4}$ between the classes of allowed operations of the $m$ single-resource theories, that is

$$
\mathcal{C}_{\text {multi }}=\bigcap_{i=1}^{m} \mathcal{C}_{i} .
$$

Notice that, alternatively, one can define the set of allowed operations $\mathcal{C}_{\text {multi }}$ as a subset of the bigger set $\cap_{i=1}^{m} \tilde{\mathcal{C}}_{i}$, where $\tilde{\mathcal{C}}_{i}$ is the set of all the CPTP maps for which $\mathcal{F}_{i}$ is invariant, see Eq. (10). When $n$ copies of the system are considered, the class of allowed operations for the multi-resource theory, $\mathcal{C}_{\text {multi, }}^{(n)}$, is obtained by the intersection between the sets of allowed operations $\mathcal{C}_{i}^{(n)}$ of the different single-resource theories, that is, $\mathcal{C}_{\text {multi }}^{(n)}=\cap_{i=1}^{m} \mathcal{C}_{i}^{(n)}$.

We can now consider the invariant sets of this multi-resource theory. Clearly, each set of free states $\mathcal{F}_{i}$ associated with the single-resource theory $\mathrm{R}_{i}$ is an invariant set for the class of operations $\mathcal{C}_{\text {multi. }}$. However, it is worth noting that the states contained in the $\mathcal{F}_{i}$ 's might not be free when the multiresource theory is considered, where a free state is (as we pointed out in the previous section) a state

\footnotetext{
${ }^{4}$ While other multi-resource theory constructions can be imagined, the one we use in this paper provides the certainty that no resource can be created out of free states.
} 
that does not contain any resource and can be realised using the allowed operations. Indeed, the states contained in the set $\mathcal{F}_{i}$ might be resourceful states for the single-resource theory $\mathrm{R}_{j}$, and therefore we would not be able to realise such states with the class of operations $\mathcal{C}_{\text {multi }}$. In Fig. 2 we show the different configurations for the invariant sets of a multi-resource theory with two resources. While in the left and central panels the theory has free states, in the right panel no free states can be found, a noticeable difference from the framework for single-resource theories.

The multi-resource theory $\mathrm{R}_{\text {multi }}$ also inherits the monotones of the single-resource theories that compose it. This follows trivially from the choice we made in defining the class of allowed operations $\mathcal{C}_{\text {multi, }}$, see Eq. (11). Furthermore, other monotones, that are only valid for the multi-resource theory, can be obtained from the ones inherited from the single-resource theories $\mathrm{R}_{i}$ 's. For example, if $f_{i}$ is a monotone for the single-resource theory $\mathrm{R}_{i}$, and $f_{j}$ is a monotone for the theory $\mathrm{R}_{j}$, their linear combination, where the linear coefficients are positive, is a monotone for the multi-resource theory

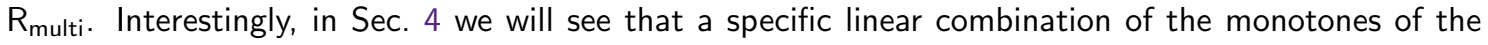
different single-resource theories plays an important role in the interconversion of resources.

Examples of multi-resource theories that can be described within our formalism are already present in the literature. In Ref. [45], for instance, the authors study the problem of state-merging when the parties can only use local operations and classical communication (LOCC), and they restrict the local operations to be incoherent operations, that is, operations that cannot create coherence (in a given basis). This theory coincides with the multi-resource theory obtained from combining two singleresource theories, the one of entanglement, whose set of allowed operations only contains quantum channels built out of LOCC, and the one of coherence, whose set of allowed operations only contains maps which do not create coherence. In this case, the structure of the invariant sets is given by the central panel of Fig. 2. Another example is the one of Ref. [35], where thermodynamics is obtained as a multi-resource theory whose class of allowed operations is a subset of the one obtained by taking the intersection of energy-non-increasing maps (operations which do not increase the average energy of the quantum system, see Sec. 3.4), and mixtures of unitary operations. In this case the resources are, respectively, average energy and entropy, and the structure of the invariant sets is given by the right panel of Fig. 2, where $\mathcal{F}_{1}$ coincides with the ground state of the Hamiltonian (if the Hamiltonian is non-degenerate), while $\mathcal{F}_{2}$ coincides with the maximally-mixed state. Other examples of multi-resource theories can be found, and in future work [59] we will present the general properties of multi-resource theories with different invariant sets structures.

\section{Reversible multi-resource theories}

In this section we study reversibility in the context of multi-resource theories. We first introduce a property, which we refer to as the asymptotic equivalence property, for multi-resource theories. We then show that, when a resource theory satisfies this property, we can (uniquely) quantify the amount of resources needed to perform an asymptotic state transformation. This allows us to introduce the notion of batteries, i.e., systems where each individual resource can be stored, and to keep track of the changes of the resources during a state transformation. Furthermore, we show that a theory which satisfies the asymptotic equivalence property is also reversible, that is, the amount of resources exchanged with the batteries during an asymptotic state transformation mapping $\rho$ into $\sigma$ is equal, with negative sign, to the amount of resources exchanged when mapping $\sigma$ into $\rho$. Finally, we show that, when the invariant sets of the theory satisfy some general properties, and the theory satisfies asymptotic equivalence, then the relative entropy distances from the different invariant sets are the unique measures of the resources. This result is a generalisation of the one obtained in single-resource theories, see Ref. [29, 32, 33].

\subsection{Asymptotic equivalence property}

Let us consider the multi-resource theory $\mathrm{R}_{\text {multi }}$ introduced in Sec. 2.2. This theory has $m$ resources, its set of allowed operations $\mathcal{C}_{\text {multi }}$ is defined in Eq. (11), and its invariant sets are the $\mathcal{F}_{i}$ 's, that is, the sets of free states of the different single-resource theories composing it. The multi-resource theory $\mathrm{R}_{\text {multi }}$ is reversible if the amount of resources spent to perform an asymptotic state transformation is equal to the amount of resources gained when the inverse state transformation is performed. In this way, performing a cyclic state transformation over the system (which recovers its initial state at the 
end of the transformation) never consumes any of the $m$ resources initially present in the system.

For a single-resource theory, the notions of reversibility and state transformation are usually associated with the rates of conversion. Suppose that we are given $n \gg 1$ copies of a state $\rho \in \mathcal{S}(\mathcal{H})$, and we want to find out the maximum number of copies of the state $\sigma \in \mathcal{S}(\mathcal{H})$ that can be obtained by acting on the system with the allowed operations. If $k$ is the maximum number of copies of $\sigma$ achievable, then the rate of conversion is defined as $R(\rho \rightarrow \sigma)=\frac{k}{n}$, see Def. 14 in appendix A. Reversibility is then defined by asking that, for all $\rho, \sigma \in \mathcal{S}(\mathcal{H})$, the rates of conversion associated to the forward and backward state transformations are such that $R(\rho \rightarrow \sigma) R(\sigma \rightarrow \rho)=1$, see Def. 15 in the appendix. It is worth noting that, when considering rates of conversion, one is in general allowed to trace out part of the system, or to add ancillary systems in a free state. For example, being able to map $n$ copies of $\rho$ into $k$ copies of $\sigma$, with $n<k$, implies that we have the possibility to add $k-n$ copies in a free state to the initial $n$ copies of $\rho$, and to act globally to produce $k$ copies of $\sigma$. This is certainly possible for single-resource theories, where free states always exists, but not always possible for multi-resource theories, see the invariant set structure of the right panel of Fig. 2.

Due to the possible absence of free states in a generic multi-resource theory, we first need to introduce the following definition ${ }^{5}$, which will then allow us to study reversibility.

Definition 1. Consider a multi-resource theory $R_{\text {multi. }}$ We say that $R_{\text {multi }}$ satisfies the asymptotic equivalence property with respect to the set of monotones $\left\{f_{i}\right\}_{i=1}^{m}$, where each $f_{i}$ is a monotone for the corresponding single-resource theory $R_{i}$ whose regularisation is not identically zero, if for all $\rho, \sigma \in \mathcal{S}(\mathcal{H})$ we have that the following two statements are equivalent,

- $f_{i}^{\infty}(\rho)=f_{i}^{\infty}(\sigma)$ for all $i=1, \ldots, m$.

- There exist a sequence of maps $\left\{\tilde{\mathcal{E}}_{n}: \mathcal{S}\left(\mathcal{H}^{\otimes n}\right) \rightarrow \mathcal{S}\left(\mathcal{H}^{\otimes n}\right)\right\}_{n}$ such that

$$
\lim _{n \rightarrow \infty}\left\|\tilde{\mathcal{E}}_{n}\left(\rho^{\otimes n}\right)-\sigma^{\otimes n}\right\|_{1}=0
$$

as well as a sequence of maps performing the reverse process. The maps $\left\{\tilde{\mathcal{E}}_{n}\right\}$ are defined as

$$
\tilde{\mathcal{E}}_{n}(\cdot)=\operatorname{Tr}_{A}\left[\mathcal{E}_{n}\left(\cdot \otimes \eta_{n}^{(A)}\right)\right],
$$

where $A$ is an ancilla composed by a sub-linear number $o(n)$ of copies of the system, and it is described by an arbitrary state $\eta_{n}^{(A)} \in \mathcal{S}\left(\mathcal{H}^{\otimes o(n)}\right)$, such that $f_{i}\left(\eta_{n}^{(A)}\right)=o(n)$ for all $i=1, \ldots, m$. The $\operatorname{map} \mathcal{E}_{n} \in \mathcal{C}_{\text {multi }}^{(n+o(n))}$ is an allowed operation of the multi-resource theory.

Here, $f_{i}^{\infty}$ is the regularisation of the monotone $f_{i},\|\cdot\|_{1}$ is the trace norm, define as $\|O\|_{1}=\operatorname{Tr}\left[\sqrt{O^{\dagger} O}\right]$ for $O \in \mathcal{B}(\mathcal{H})$, and we are using the little-o notation, where $g(n)=o(n)$ means $\lim _{n \rightarrow \infty} \frac{g(n)}{n}=0$.

An example of a multi-resource theory that satisfies the above property is thermodynamics (even in the case in which multiple conserved quantities are present), as shown in Refs. [35, 36]. In this example the monotones for which asymptotic equivalence is satisfied are the average energy and the Von Neumann entropy of the system. Notice that the above property implicitly assumes that the monotones $f_{i}$ 's can be regularised, that is, that the limit involved in the regularisation is always finite. Furthermore, in this property we are allowing the agent to act over many copies of the system with more than just the set of allowed operations; we assume the agent to be able to use a small ancillary system, sub-linear in the number of copies of the main system. Roughly speaking, the role of this ancilla is to absorb the fluctuations in the monotones $f_{i}^{\infty}$ 's during the asymptotic state transformation. It is important to notice that this ancillary system only contributes to the transformation by exchanging a sub-linear amount of resources. Thus, its contribution per single copy of the system is negligible when $n \gg 1$, which justifies the use of this additional tool.

The asymptotic equivalence property essentially states that the multi-resource theory can reversibly map between any two states with the same values of the monotones $f_{i}$ 's. In particular, transforming between such two states comes at no cost, since we can do so by using the allowed operations of the

\footnotetext{
${ }^{5}$ Notice that this definition is analogous to the notion of "seed regularisation" in Ref. [23, Sec. 6], although in our case we are solely focused on reversible transformations and on equalities of monotones.
} 
theory, $\mathcal{C}_{\text {multi. }}$ It is worth noting that, when the number of considered resources is $m=1$, that is, our theory is a single-resource theory, the notion of asymptotic equivalence given in Def. 1 corresponds to the one given in terms of rates of conversion, Def. 15 . We prove this equivalence in appendix $A$, see Thm. 18. The set of monotones in Def. 1 is not a priori unique; however, in the following section we identify the properties that the monotones need to satisfy for this set to be unique, see Thm. 5. Finally, notice that the asymptotic equivalence property does not say anything about the state transformations which involve states with different values of the monotones $f_{i}$ 's. To include these transformations in the theory, we will have to add a bit more structure to the current framework, by considering some additional systems that can store a single type of resource each, which we refer to as batteries [60].

\subsection{Quantifying resources with batteries}

When a multi-resource theory satisfies the asymptotic equivalence property of Def. 1, we have that states with the same values of a specific set of monotones can be inter-converted between each others. In this section, we show that these monotones actually quantify the amount of resources contained in the system. To do so, we need to introduce some additional systems, which can only store a single kind of resource each, and can be independently addressed by the agent. These additional systems are referred to as batteries. Let us suppose that the multi-resource theory $R_{\text {multi }}$ satisfies the asymptotic equivalence property with respect to the set of monotones $\left\{f_{i}\right\}_{i=1}^{m}$, and that the quantum system over which the theory acts is actually divided into $m+1$ partitions. The first partition is the main system $S$, and the remaining ones are the batteries $B_{i}$ 's. Then, the Hilbert space under consideration is $\mathcal{H}=\mathcal{H}_{S} \otimes \mathcal{H}_{B_{1}} \otimes \ldots \otimes \mathcal{H}_{B_{m}}$.

Let us now introduce some properties the monotones need to satisfy in order for the resources to be quantified in a meaningful way. Since each resource is associated to a different monotone, we can forbid a battery to store more than one resource by constraining the set of states describing it to those ones with a fixed value of all but one monotones.

M1 Consider two states $\omega_{i}, \omega_{i}^{\prime} \in \mathcal{S}\left(\mathcal{H}_{B_{i}}\right)$ describing the battery $B_{i}$. Then, the value of the regularisation of any monotone $f_{j}$ (where $j \neq i$ ) over these two states is fixed,

$$
f_{j}^{\infty}\left(\omega_{i}^{\prime}\right)=f_{j}^{\infty}\left(\omega_{i}\right), \quad \forall j \neq i .
$$

In this way, the battery $B_{i}$ is only able to store and exchange the resource associated with the monotone $f_{i}$. It would be natural to extend the condition of Eq. (14) to the monotones themselves, rather than to use their regularisations. However, this condition is not required for deriving our results, and to use it in our proofs we would need an additional assumption, namely the additivity of the monotones.

In order to address each battery as an individual system, we ask the value of the monotones over the global system to be given by the sum of their values over the individual components,

M2 The regularisations of the monotones $f_{i}$ 's can be separated between main system and batteries,

$$
f_{i}^{\infty}\left(\rho \otimes \omega_{1} \otimes \ldots \otimes \omega_{m}\right)=f_{i}^{\infty}(\rho)+f_{i}^{\infty}\left(\omega_{1}\right)+\ldots+f_{i}^{\infty}\left(\omega_{m}\right),
$$

where $\rho \in \mathcal{S}\left(\mathcal{H}_{S}\right)$ is the state of the main system, and $\omega_{i} \in \mathcal{S}\left(\mathcal{H}_{B_{i}}\right)$ is the state of the battery $B_{i}$.

The above property allows us to separate the contribution given by each subsystem to the amount of $i$-th resource present in the global system. It is important to stress that we are here requiring additivity for the regularisation of the monotones between system and batteries, and between batteries, but we are not requiring the regularised monotones to be additive in general.

We then ask the monotones to satisfy an additional property, so as to simplify the notation. Namely, we ask the zero of each monotone $f_{i}$ to coincide with its value over the states in $\mathcal{F}_{i}$,

M3 For each $n \in \mathbb{N}$ and $i \in\{1, \ldots, m\}$, the monotone $f_{i}$ is equal to 0 when computed over the states of $\mathcal{F}_{i}^{(n)}$, that is

$$
f_{i}\left(\gamma_{i, n}\right)=0, \quad \forall \gamma_{i, n} \in \mathcal{F}_{i}^{(n)} .
$$


This property serves as a way to "normalise" the monotone, setting its value to 0 over the states that were free for the specific single-resource theory the monotone is linked to. Notice that property M3 is trivially satisfied by any monotone after a translation. The next property requires that tracing out part of the system does not increase the value of the monotones $f_{i}$ 's,

M4 For all $n, k \in \mathbb{N}$ where $k<n$, the monotones $f_{i}$ 's are such that

$$
f_{i}\left(\operatorname{Tr}_{k}\left[\rho_{n}\right]\right) \leq f_{i}\left(\rho_{n}\right), \quad \forall i \in\{1, \ldots, m\}
$$

where $\rho_{n} \in \mathcal{S}\left(\mathcal{H}^{\otimes n}\right)$ and $\operatorname{Tr}_{k}\left[\rho_{n}\right] \in \mathcal{S}\left(\mathcal{H}^{\otimes n-k}\right)$.

This property implies that the resources contained in a system cannot increase if we discard/forget part of it.

We require our monotones to satisfy sub-additivity, namely

M5 For all $n, k \in \mathbb{N}$, the monotones $f_{i}$ 's are such that

$$
\begin{aligned}
& \qquad f_{i}\left(\rho_{n} \otimes \rho_{k}\right) \leq f_{i}\left(\rho_{n}\right)+f_{i}\left(\rho_{k}\right), \quad \forall i \in\{1, \ldots, m\} . \\
& \text { where } \rho_{n} \in \mathcal{S}\left(\mathcal{H}^{\otimes n}\right) \text { and } \rho_{k} \in \mathcal{S}\left(\mathcal{H}^{\otimes k}\right) .
\end{aligned}
$$

That is, the amount of resources contained in two uncorrelated systems, when measured on the two systems independently, is bigger or equal to the value measured on the two systems together. This is the case, for example, of the relative entropy of entanglement [61]. Notice that sub-additivity is here explicity required since, as we stressed before, property $\mathrm{M} 2$ only requires additivity between system and different batteries, but not between different partitions of the individual system or battery. Another property we require is for the monotones $f_{i}$ 's to be sub-extensive,

M6 Given any sequence of states $\left\{\rho_{n} \in \mathcal{S}\left(\mathcal{H}^{\otimes n}\right)\right\}$, the monotones $f_{i}$ 's are such that

$$
f_{i}\left(\rho_{n}\right)=O(n), \quad \forall i \in\{1, \ldots, m\} .
$$

where we are using the big-O notation.

This property is satisfied, for example, if the monotones scale extensively, that is, if they scale linearly in the number of systems considered. In the next section we will encounter a family of monotones which indeed satisfy this property, namely the relative entropy distance from a given set of free states, when some fairly generic conditions are satisfied by such set (see Prop. 6). However, it is worth noting that property M6 is not equivalent to extensivity, since a monotone scaling sub-linearly in the number of systems would still satisfy it. We demand that our monotones satisfy this property so as to be able to regularise them (although their regularisation might be identically zero on the whole state space). The last property we ask concerns a particular kind of continuity the monotones need to satisfy,

M7 The monotones $f_{i}$ 's are asymptotic continuous, that is, for all sequences of states $\rho_{n}, \sigma_{n} \in \mathcal{S}\left(\mathcal{H}^{\otimes n}\right)$ such that $\left\|\rho_{n}-\sigma_{n}\right\|_{1} \rightarrow 0$ for $n \rightarrow \infty$, where $\|\cdot\|_{1}$ is the trace norm, we have

$$
\frac{\left|f_{i}\left(\rho_{n}\right)-f_{i}\left(\sigma_{n}\right)\right|}{n} \rightarrow 0 \text { for } n \rightarrow \infty, \quad \forall i \in\{1, \ldots, m\}
$$

This notion of asymptotic continuity coincides with condition (C2) given in Ref. [62].

This property implies that the monotones are physically meaningful, since their values over sequences of states converge if the sequences of states converge asymptotically. In Thm. 5 we show that, when the monotones satisfy asymptotic continuity, they are the unique quantifiers of the amount of resources contained in the main system.

We can now use this formalism to discuss how resources can be quantified in a multi-resource theory, and consequently how the asymptotic equivalence property implies that the theory is reversible. Let us consider any two states $\rho, \sigma \in \mathcal{S}\left(\mathcal{H}_{S}\right)$, that do not need to have the same values for the monotones $f_{i}$ 's. Then, we choose the initial and final states of each battery $B_{i}$ such that

$$
f_{i}^{\infty}\left(\rho \otimes \omega_{1} \otimes \ldots \otimes \omega_{m}\right)=f_{i}^{\infty}\left(\sigma \otimes \omega_{1}^{\prime} \otimes \ldots \otimes \omega_{m}^{\prime}\right), \quad \forall i=1, \ldots, m,
$$


where $\omega_{i}, \omega_{i}^{\prime} \in \mathcal{S}\left(\mathcal{H}_{B_{i}}\right)$, for $i=1, \ldots, m$. Under these conditions, due to the asymptotic equivalence property of $\mathrm{R}_{\text {multi }}$, we have that the two global states can be asymptotically mapped one into the other in a reversible way, using the allowed operations of the theory, that is

$$
\rho \otimes \omega_{1} \otimes \ldots \otimes \omega_{m} \stackrel{\text { asympt }}{\longleftrightarrow} \sigma \otimes \omega_{1}^{\prime} \otimes \ldots \otimes \omega_{m}^{\prime},
$$

where the symbol $\stackrel{\text { asympt }}{\longleftrightarrow}$ means that there exists two allowed operations that maps $n \gg 1$ copies of the state on the lhs into the state of the rhs, and viceversa, while satisfying the condition in the second statement of Def. 1.

We can now properly define the notion of resources in this framework. The resource associated with the monotone $f_{i}$ is the one exchanged by the battery $B_{i}$ during a state transformation.

Definition 2. Consider a multi-resource theory $R_{\text {multi }}$ with $m$ resources, satisfying the asymptotic equivalence property with respect to the set of monotones $\left\{f_{i}\right\}_{i=1}^{m}$. For a state transformation of the form given in Eq. (22), we define the amount of $i$-th resource exchanged between the system $S$ and the battery $B_{i}$ as

$$
\Delta W_{i}:=f_{i}^{\infty}\left(\omega_{i}^{\prime}\right)-f_{i}^{\infty}\left(\omega_{i}\right),
$$

where $\omega_{i}, \omega_{i}^{\prime} \in \mathcal{S}\left(\mathcal{H}_{B_{i}}\right)$ are, respectively, the initial and final state of the battery $B_{i}$.

It is now possible to compute the amount of the $i$-th resource $\Delta W_{i}$ needed to map the state of the main system $\rho$ into $\sigma$.

Proposition 3. Consider a theory $R_{\text {multi }}$ with $m$ resources and allowed operations $\mathcal{C}_{\text {multi }}$, equipped with batteries $B_{1}, \ldots, B_{m}$. If the theory satisfies the asymptotic equivalence property with respect to the set of monotones $\left\{f_{i}\right\}_{i=1}^{m}$, and these monotones satisfy the properties M1 and M2, then the amount of $i$-th resource needed to perform the asymptotic state transformation $\rho \rightarrow \sigma$ is equal to

$$
\Delta W_{i}=f_{i}^{\infty}(\rho)-f_{i}^{\infty}(\sigma) .
$$

Proof. Due to asymptotic equivalence, a transformation mapping the global state $\rho \otimes \omega_{1} \otimes \ldots \otimes \omega_{m}$ into $\sigma \otimes \omega_{1}^{\prime} \otimes \ldots \otimes \omega_{m}^{\prime}$ exists iff the conditions in Eq. (21) are satisfied. For a given $i$, using the property $\mathrm{M} 2$ of the monotone $f_{i}$, we can re-write the condition as

$$
f_{i}^{\infty}(\rho)+f_{i}^{\infty}\left(\omega_{1}\right)+\ldots+f_{i}^{\infty}\left(\omega_{m}\right)=f_{i}^{\infty}(\sigma)+f_{i}^{\infty}\left(\omega_{1}^{\prime}\right)+\ldots+f_{i}^{\infty}\left(\omega_{m}^{\prime}\right) .
$$

Then, we can use the property $M 1$, which guarantees that the only systems for which $f_{i}$ changes are the main system and the battery $B_{i}$. Thus, we find that

$$
f_{i}^{\infty}(\rho)+f_{i}^{\infty}\left(\omega_{i}\right)=f_{i}^{\infty}(\sigma)+f_{i}^{\infty}\left(\omega_{i}^{\prime}\right) .
$$

By rearranging the factors in the above equation, and using the definition of $\Delta W_{i}$ given in Eq. (23), we prove the proposition.

It is now easy to show that, if $\mathrm{R}_{\text {multi }}$ satisfies the asymptotic equivalence property, any state transformation on the main system $S$ is reversible. Indeed, from Eq. (24) it follows that the amount of resources used to map the state of this system from $\rho$ to $\sigma$ is equal, but with negative sign, to the amount of resources used to perform the reverse transformation, from $\sigma$ to $\rho$. Therefore, any cyclic state transformation over the main system leaves the amount of resources contained in the batteries unchanged.

The above formalism also provides us with a way to quantify the amount of resources contained in the main system. Indeed, if the system is described by the state $\rho \in \mathcal{S}\left(\mathcal{H}_{S}\right)$, the amount of $i$-th resource contained in the system is given by the amount of $i$-th resource exchanged, $\Delta W_{i}$, while mapping $\rho$ into a state contained in $\mathcal{F}_{i}$. Using property M3 and Prop. (24) it follows that

Corollary 4. Consider a theory $R_{\text {multi }}$ with $m$ resources and allowed operations $\mathcal{C}_{\text {multi, equipped with }}$ batteries $B_{1}, \ldots, B_{m}$. If the theory satisfies the asymptotic equivalence property with respect to the set of monotones $\left\{f_{i}\right\}_{i=1}^{m}$, and these monotones satisfy the properties $M 1, M 2$, and $M 3$, then the amount of the $i$-th resource contained in the main system, when described by the state $\rho$, is given by $f_{i}^{\infty}(\rho)$. 


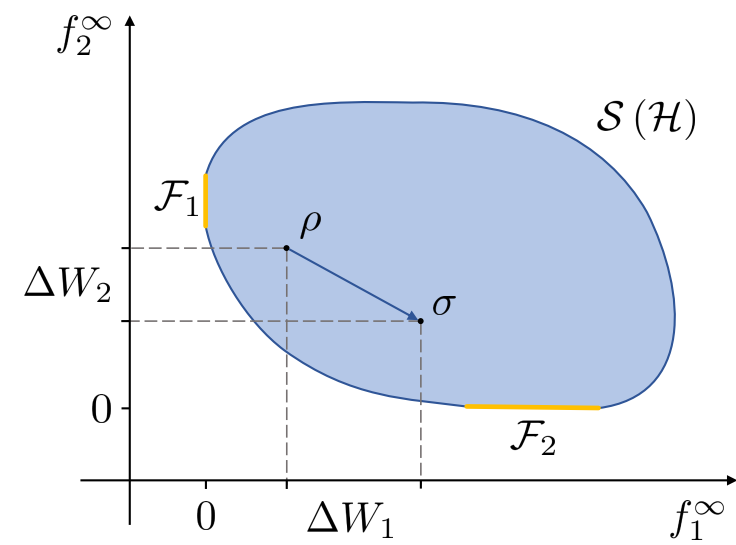

Figure 3: In the figure we represent the state-space $\mathcal{S}(\mathcal{H})$ of a multi-resource theory $\mathrm{R}_{\text {multi }}$ with two resources. In order for the diagram to be a meaningful representation of this state-space, we need the theory to satisfy the asymptotic equivalence property of Def. 1 with respect to the monotones $f_{1}$ and $f_{2}$. In fact, when the theory satisfies this property we can divide $\mathcal{S}(\mathcal{H})$ into equivalence classes of states with the same value of the regularised monotones $f_{1}^{\infty}$ and $f_{2}^{\infty}$, which become the abscissa and ordinate of the diagram. The state-space of the theory is represented by the blue region, and the yellow segments are the invariant sets $\mathcal{F}_{1}$ and $\mathcal{F}_{2}$. These sets are disjoint, since the two segments do not intercept each other, and the resource theory $\mathrm{R}_{\text {multi }}$ thus corresponds to the one depicted in the right panel of Fig. 2. Two equivalence classes, respectively associated to the states $\rho$ and $\sigma$, are represented in the diagram. The amount of resources that is exchanged when transforming from one state to the other, Eq. (24), is given in the diagram by the difference between the coordinates of these two points. Notice that, for ease of viewing, we have shifted the origin of the axis.

It is worth noting that, in general, one cannot extract all the resources contained in the main system at once. Indeed, this is only possible when the multi-resource theory contains free states, like for example in the cases depicted in the left and centre panels of Fig. 2. Furthermore, the process of resources extraction is in general non-trivial, since property $\mathrm{M} 1$ forbids each battery from storing more than one kind of resource. As a result, it is not possible to simply perform a swap operation which exchanges the state of the system with one of the batteries, see Sec. 5.2 for an example involving the theory of local control.

Being able to quantify the amount of resources contained in a given quantum state allows us to represent the whole state-space of the theory in a resource diagram $[23,35]$. In fact, from the definition of asymptotic equivalence it follows that, if two states contain the same amount of resources, i.e., if they have the same values of the monotones $f_{i}^{\infty}$ 's, then we can map between them using the allowed operations $\mathcal{C}_{\text {multi. }}$. This property implies that we can divide the entire state-space into equivalence classes, that is, sets of states with same value of the $m$ monotones (where we recall that $m$ is the number of resources, or batteries, in the theory). Then, we can represent each equivalence class as a point in a $m$-dimensional diagram, with coordinates given by the values of the monotones. By considering all the different equivalence classes, we can finally represent the state-space of the main system in the diagram, see for example Fig. 3, where the state-space of a two-resource theory is shown.

\subsection{Reversibility implies a unique measure for each resource}

We now show that, when a multi-resource theory satisfies the asymptotic equivalence property with respect to a set of monotones $\left\{f_{i}\right\}_{i=1}^{m}$, and these monotones satisfy the properties $\mathrm{M} 1-\mathrm{M} 7$, then there exists a unique quantifier for each resource contained in the main system. In particular, when the $i$-th resource is considered, this quantifier coincides with $f_{i}^{\infty}$, modulo a multiplicative factor which sets the scale. It is worth noting that such multiplicative factor can be different for each resource. Indeed, the resources are generally independent of each other, since they are quantified by different measures. Each measure can have a different unit, which corresponds to an individual rescaling factor applied to each resource measure independently.

In the previous section, Cor. 4, we showed that a quantifier exists if the monotones satisfy the first three properties M1, M2, and M3. However, when these monotones are also asymptotic continuous, 
property M7, we can prove that they uniquely quantify the amount of resources contained in the main system. This means that one cannot find other monotones $g_{i}$ 's that give the same equivalence classes of the $f_{i}$ 's, but order them in a different way. Asymptotic continuity was used in Ref. [32] to show that the relative entropy distance from the set of free states of a reversible single-resource theory is the unique measure of resource. Thus, the following theorem (whose proof can be found in appendix D.1) can be understood as a generalisation of the above result to multi-resource theories,

Theorem 5. Consider the resource theory $R_{\text {multi }}$ with $m$ resources, equipped with the batteries $B_{i}$ 's, where $i=1, \ldots, m$. Suppose the theory satisfies the asymptotic equivalence property with respect to the set of monotones $\left\{f_{i}\right\}_{i=1}^{m}$. If these monotones satisfy the properties $M 1-M 7$, then the amount of $i$-th resource contained in the main system $S$ is uniquely quantified by the regularisation of the monotone $f_{i}$ (modulo a multiplicative constant).

In particular, we now consider the case of a multi-resource theory $\mathrm{R}_{\text {multi }}$ that satisfies the asymptotic equivalence property of Def. 1 with respect to the relative entropy distances from the invariant sets $\mathcal{F}_{i}$ 's. We refer to the relative entropy distance from the set $\mathcal{F}_{i}$ as $E_{\mathcal{F}_{i}}$, whose definition can be found in Eq. (9). Since the multi-resource theory we consider is equipped with batteries, and we want to be able to measure the amount of resources they contain independently of the other subsystems, we ask the invariant sets to be of the form

$$
\mathcal{F}_{i}=\mathcal{F}_{i, S} \otimes \mathcal{F}_{i, B_{1}} \otimes \ldots \otimes \mathcal{F}_{i, B_{m}}
$$

so that the main system $S$ and the batteries $B_{i}$ 's all have they own independent invariant sets. We now show that, under very general assumptions over the properties of the invariant sets, the regularised relative entropy distances from these sets are the unique quantifiers of the resources, provided that these quantities are not identically zero over the whole state space ${ }^{6}$. This result follows from Thm. 5 , and from the fact that these monotones satisfy the properties $M 1, M 2, M 3$, and $M 7$ listed in the previous sections. The properties we are interested in for the invariant sets $\left\{\mathcal{F}_{i}\right\}_{i=1}^{m}$ of the theory are very general, and they are satisfied in most of the known resource theories, see Refs. [31, 63].

F1 The sets $\mathcal{F}_{i}$ 's are closed sets.

F2 The sets $\mathcal{F}_{i}$ 's are convex sets

F3 Each set $\mathcal{F}_{i}$ contains at least one full-rank state.

F4 The sets $\mathcal{F}_{i}$ 's are closed under tensor product, that is, $\mathcal{F}_{i}^{(k)} \otimes \mathcal{F}_{i}^{(n)} \subseteq \mathcal{F}_{i}^{(n+k)}$ for all $i=1, \ldots, m$.

F5 The sets $\mathcal{F}_{i}$ 's are closed under partial tracing, that is, $\operatorname{Tr}_{k}\left[\mathcal{F}_{i}^{(n)}\right] \subseteq \mathcal{F}_{i}^{(n-k)}$ for all $i=1, \ldots, m$.

Let us briefly comment on the above properties. Property F1 requires that any converging sequence in the set converges to an element in the set. This property is necessary for the continuity of the resource theory. Property F2, instead, tells us that we are allowed to forget the exact state describing the system, and therefore we can have mixture of states. Property F3 is necessary for the relative entropy distance to be physically natural, since the quantity $D(\rho \| \sigma)$, see Eq. (8), diverges when $\operatorname{supp}(\rho) \nsubseteq \operatorname{supp}(\sigma)$. Finally, property F4 implies that composing two systems that do not contain any amount of $i$-th resource is not going to increase that resource, and similarly, property F5 implies that forgetting about part of a system which does not contain resources will not create resources.

When the invariant sets satisfy the above properties, the relative entropy distances $E_{\mathcal{F}_{i}}$ 's satisfy the same properties discussed in the previous section,

Proposition 6. Consider a resource theory $R_{\text {multi }}$ with $m$ resources, equipped with the batteries $B_{i}$ 's, where $i=1, \ldots, m$. Suppose the class of allowed operations is $\mathcal{C}_{\text {multi }}$ and the invariant sets are $\left\{\mathcal{F}_{i}\right\}_{i=1}^{m}$. If the invariant set $\mathcal{F}_{i}$ is of the form of Eq. (27), and it satisfies the properties $F 1-F 5$, then the relative entropy distances from this set, $E_{\mathcal{F}_{i}}$, is a regularisable monotone under the class of allowed operations, and it obeys the properties M1 - M7.

\footnotetext{
${ }^{6}$ An example where the regularised relative entropy from an invariant set is identically zero for all states in $\mathcal{S}(\mathcal{H})$ is the resource theory of asymmetry, see Ref. [15].
} 
This result is known in the literature, see Refs. [63, 64], but we nevertheless provide a proof in appendix D.2 to make the paper self-contained. By virtue of Thm. 5 it then follows that, if $E_{\mathcal{F}_{i}}^{\infty}$ has a positive value over the states that are not in $\mathcal{F}_{i}$, then it is the unique quantifier of the amount of $i$-th resource contained in the system for a multi-resource theory that satisfies the asymptotic equivalence property with respect to these monotones. Furthermore, the amount of $i$-th resource used to map the main system from the state $\rho$ into the state $\sigma$ is then equal to

$$
\Delta W_{i}=E_{\mathcal{F}_{i}}^{\infty}(\rho)-E_{\mathcal{F}_{i}}^{\infty}(\sigma)
$$

for all $i=1, \ldots, m$.

\subsection{Relaxing the conditions on the monotones}

There are situations, when we consider specific resource theories, in which some of the properties of the set of free states are not satisfied. In particular, we can have that the set of free states does not contain a full-rank state, that is, property F3 is not satisfied. An example would be the resource theory of energy-non-increasing maps for a system with Hamiltonian $H$,

$$
\mathcal{C}_{H}=\left\{\mathcal{E}_{H}: \mathcal{B}(\mathcal{H}) \rightarrow \mathcal{B}(\mathcal{H}) \mid \operatorname{Tr}\left[\mathcal{E}_{H}(\rho) H\right] \leq \operatorname{Tr}[\rho H] \forall \rho \in \mathcal{S}(\mathcal{H})\right\} .
$$

An example of a subset of $\mathcal{C}_{H}$ are unitary operations which commute with the Hamiltonian $H$ (as in the resource theory of Thermal Operations). If the Hamiltonian $H$ has a non-degenerate ground state $|g\rangle$, then it is easy to show that this state is fixed, that is,

$$
\mathcal{E}_{H}(|\mathrm{~g}\rangle\langle\mathrm{g}|)=|\mathrm{g}\rangle\langle\mathrm{g}|
$$

In fact, the operation $\mathcal{E}_{\mathrm{g}}(\cdot)=\operatorname{Tr}_{A}\left[S\left(\cdot \otimes|\mathrm{g}\rangle\left\langle\left.\mathrm{g}\right|_{A}\right) S^{\dagger}\right]\right.$, where $S$ is the unitary operation implementing the swap between the two states, belongs to $\mathcal{C}_{H}$ and maps all states into the ground state. Thus, the set of free states does not contain a full-rank state, which implies that the relative entropy distance from this set is ill-defined, and it is not asymptotic continuous. Notice that the above argument holds even in the case of a degenerate ground state, with the difference that the invariant set would be composed by any state with support on this degenerate subspace.

We can introduce a different monotone for this kind of resource theory, that is, the average of the observable which is not increased by the allowed operations (modulo a constant factor). For the example we are considering, this monotone would be

$$
M_{H}(\rho)=\operatorname{Tr}[H \rho]-E_{\mathrm{g}},
$$

where $H$ is the Hamiltonian of the system, and $E_{\mathrm{g}}=\operatorname{Tr}[H|\mathrm{~g}\rangle\langle\mathrm{g}|]$ is the energy of the ground state. When $n$ copies of the system are considered, we define the total Hamiltonian as $H_{n}=\sum_{i=1}^{n} H^{(i)}$, where $H^{(i)}$ is the Hamiltonian acting on the $i$-th copy. In this case, it is easy to show that this quantity is equal to 0 when evaluated on the fixed state $|\mathrm{g}\rangle\langle\mathrm{g}|$, property $\mathrm{M} 3$, is monotonic under partial tracing, property $\mathrm{M} 4$, is additive (and therefore satisfies sub-additivity, property M5), and it scales extensively in the number of copies of the system, thus satisfying property M6. Furthermore, $M_{H}(\cdot)$ is monotonic under the class of operations (by definition of the class itself), and it is asymptotic continuous, property M7, as shown in Prop. 22 in appendix D.2. If batteries are introduced, we can define the operator $H$ is such a way that properties M1 and M2 are satisfied, see for example Sec. 5.1.

Thus, if one (or more) of the monotones of the multi-resource theory is of the form given in Eq. (31), we have that the results of the previous section still apply, particularly Thm. 5. Furthermore, we can quantify the change in the resource associated with $M_{H}$ during a state transformation $\rho \rightarrow \sigma$ with Eq. (28), where the regularised relative entropy distance $E_{\mathcal{F}_{i}}^{\infty}$ is replaced with the regularised monotone $M_{H}^{\infty}$. As a side remark, we notice that the monotone $M_{H}$ can be obtained as

$$
M_{H}(\rho)=\lim _{\beta \rightarrow \infty} \frac{1}{\beta} D\left(\rho \| \tau_{\beta}\right),
$$

where $\tau_{\beta}=e^{-\beta H} / Z$ is the Gibbs state of the Hamiltonian $H$, and $Z=\operatorname{Tr}\left[e^{-\beta H}\right]$ is the partition function of the system. 


\section{Bank states, interconversion relations, and the first law}

Within certain types of multi-resource theories, it is possible to inter-convert the resources stored in the batteries, i.e., to exchange one resource for another at a given exchange rate. Examples of resource interconversion can be found in thermodynamics, where Landauer's principle [65] tells us that energy can be exchanged for information, while a Maxwell's demon can trade information for energy [66]. In these examples, a thermal bath is necessary to perform the interconversion of resources. Indeed, in the following sections we show that in order to exchange between resources one always needs an additional system, which we refer to as a bank, that captures the necessary properties of thermal baths in thermodynamics, and abstracts them so that they can be applied to other resource theories. When such a system exists, we can pay a given amount of one resource and gain a different amount of another resource, with an exchange rate that only depends on the state describing the bank, see Thm. 9. Within the thermodynamic examples we are considering, this corresponds to exchanging one bit of information for one unit of energy, and vice versa. The exchange rate of these processes is proportional to the temperature of the thermal bath.

During a resource interconversion the state of the bank should not change its main properties, so that we can keep using it indefinitely. Furthermore, we should always have to invest one resource in order to gain the other. For these reasons the bank is taken to be of infinite size, and its state to be passive, i.e., to always contain the minimum possible values of the resources. In fact, in the thermodynamic examples we are considering, the thermal bath has infinite size, and its state has maximum entropy for fixed energy, or equivalently minimum energy for fixed entropy [67]. We additionally show that the relative entropy distance from the set of bank states plays a fundamental role in quantifying the exchange rate at which resources are inter-converted, see Cor. 12. For instance, in thermodynamics this quantity is proportional to the Helmholtz free energy $F=E-T S$, which links together the two resources, internal energy $E$ and information, which is proportional to $-S$. Through this quantity, one can define the exchange rate between energy and entropy, i.e., the temperature of the thermal bath $T$. Finally, we introduce a first-law-like relation for multi-resource theories. The first law consists of a single relation that regulates the state transformation of a system when the agent has access to a bank for exchanging the resources. In particular, this relation links the change in the relative entropy distance from the set of bank states over the main system to the amount of resources exchanged by the batteries during the transformations, see Cor. 13. In the example we are considering, this relation coincides with the First Law of thermodynamics, as it connects a change in the Helmholtz free energy $\Delta F$ of the system with the energy and information exchanged by the batteries,

$$
\Delta F=\Delta W_{E}+T \Delta W_{I},
$$

where $\Delta W_{E}$ is the energy exchanged by the first battery, $\Delta W_{I}$ is the information exchanged by the second battery, and $T$ is the background temperature, describing the state of the bank.

We now briefly discuss about the value that resources have in the different theories of thermodynamics, and the role of the first law in connecting these resources together. Let us first consider the single-resource theory of thermodynamics, where the system is in contact with an infinite thermal reservoir [10]. To perform a state transformation we need to provide only one kind of resource, known as athermality $(\Delta F)$, or work. Since the thermal reservoir is present, it is easy to get close to the free state, i.e. to the thermal state at temperature $T$, because we can simply thermalise the system with the allowed operations. However, it is difficult to go in the opposite direction, unless we use part of the athermality stored in a battery. For this reason, a positive increment in the athermality of the battery is considered valuable, while a negative change is considered a cost.

Let us now move to the multi-resource theory of thermodynamics, whose allowed operations are energy-preserving unitary operations [35]. In this case, it is easy to see that negative and positive contributions of energy and information are equally valuable, since these two quantities are conserved by the set of allowed operations. As a result, the agent cannot perform state transformations in any direction without having access to the batteries. If we now allow the agent to use a thermal bath as a bank, and we keep the system decoupled from it (so that the agent cannot perform operations that thermalise the system for free), we find that changing a single resource, either energy or information, is enough to perform a generic state transformation on the system. In fact, we can always inter-convert one resource for the other with the bank, and then change the state of the system accordingly. Notice that, however, we still have that negative and positive change in one resource are equally valuable. 
Thus, it seems that the advantage that multi-resource theories provide over single-resource theories is that they make explicit which resources are used during a state transformation. And the link between the single resource and the multiple ones is given by the first law. In thermodynamics, for example, we have that the first law, Eq. (33), indicates that the amount of athermality $\Delta F$ needed to transform a state can be actually divided in two contributions, energy $\Delta W_{E}$ and information $\Delta W_{I}$. Notice that all of these quantities can be understood in terms of the relative entropy distance to an invariant set of states. Athermality being measured by its relative entropy distance to the thermal state, information and energy being the relative entropy to the maximally mixed or ground state. As we will see, the generalised first law given in Eq. (45) also relates the relative entropy to the bank state, to the relative entropies to the invariant sets of the single resource theories.

\subsection{Banks and interconversion of resources}

We now introduce the bank system, and show how this additional tool allows us to perform interconversion between resources. To simplify the notation, we only focus on a theory with two resources. However, the results we obtain also apply to theories with more resources, since in that case we can just select two resources and perform interconversion while keeping the others fixed. Thus, in the following we consider a resource theory $\mathrm{R}_{\text {multi }}$ with two invariant sets $\mathcal{F}_{1}$ and $\mathcal{F}_{2}$ (each of them associated with one of the resources), and allowed operations $\mathcal{C}_{\text {multi }}$. We assume the theory to satisfy the asymptotic equivalence property of Def. 1 with respect to the relative entropy distances from $\mathcal{F}_{1}$ and $\mathcal{F}_{2}$, and we ask the two invariant sets to satisfy the properties F1, F2, and F3, while we replace properties F4 and F5 with the following, more demanding, property

F5b The invariant sets $\mathcal{F}_{i}$ 's are such that $\mathcal{F}_{i}^{(n)}=\mathcal{F}_{i}^{\otimes n}$, for all $n \in \mathbb{N}$.

The above properties implies that the relative entropy distances $E_{\mathcal{F}_{1}}$ and $E_{\mathcal{F}_{2}}$ are the unique quantifiers for the two resources of our theory, as we have seen in Sec. 3.3. From property F5b it follows that these two monotones are additive, i.e., $E_{\mathcal{F}_{i}}(\rho \otimes \sigma)=E_{\mathcal{F}_{i}}(\rho)+E_{\mathcal{F}_{i}}(\sigma)$ for $i=1,2$, and consequently that their regularisation $E_{\mathcal{F}_{i}}^{\infty}$ coincides with $E_{\mathcal{F}_{i}}$. Furthermore, the properties F2 and F5b together imply that the invariant sets are composed by a single state, i.e., $\mathcal{F}_{i}=\left\{\rho_{i}\right\}$, where $\rho_{i} \in \mathcal{S}(\mathcal{H})$, for $i=1,2$. We make use of property F5b in Lem. 23, shown in appendix D.2, which itself is used to prove some essential properties of the set of bank states, see Def. 7 . This property is ultimately used to show that the exchange rate between resources is given by the relative entropy distance from the set of states describing the bank, see Cor. 12.

It is important to stress that property F5b is not satisfied by every multi-resource theory. For example, this property is satisfied by the multi-resource theory of thermodynamics, but it is violated by other theories, like entanglement theory, where the set of free states is composed of separable states. We are currently working to weaken this property, following the ideas presented in Ref. [63], by requiring the invariant sets to be closed under permutations of copies. This less demanding property should allow us to use the approximate de Finetti's theorems [68], and to obtain similar conditions to those obtained with F5b. To study the interconversion of entanglement with some other resource, however, one can think of restricting the state space of the theory in a way in which the resulting subset of separable states satisfies property F5b, see the example in Sec. 5.2. Finally, it is worth noting that all the results we obtain in this section also apply if one of the monotones, or both, is of the form shown in Eq. (31). Indeed, these monotones satisfy the same properties of the relative entropy distances, with the difference that the corresponding invariant set can be composed by multiple states, and these states do not need to have full rank.

Let us now consider an example of resource interconversion which will highlight the properties that we are searching for in a bank system. Suppose we have a certain amount of euros and pounds in our wallet, and we want to convert one into the other, for example, from pounds to euros. In order to convert these two currencies we need to go to the bank, that we would expect to satisfy the following properties. The first property could be referred to as passivity of the bank, and it is represented by the fact that if we do not provide some pounds, we cannot receive any euros (and vice versa). Second is the existence of an exchange rate, that is, the bank will convert the two currency at a certain exchange rate, and this rate can be different depending on the bank we use. The last property concerns the catalytic nature of the bank, since we would like a bank not to change the exchange rate between pounds and 
euros as a consequence of our transaction (this last property is approximately satisfied by real banks, at least for the amount exchanged by average costumers).

The previous example shows that, in order to achieve resource interconversion, we need to introduce in our framework an additional system, the bank, with some specific properties. Within our formalism, we consider the same multi-partite system introduced in Sec. 3.2, with the main system $S$, and two batteries $B_{1}$ and $B_{2}$. The system $S$ is now used as a bank, which has to satisfy the three essential properties (passivity, existence of a rate, catalytic behaviour) that we have informally described in the previous paragraph, and that we are going to formalise in the following. First of all, we need the states describing the bank to be passive, meaning that we should not be able to extract from this system both resources at the same time, since we always need to pay one resource to gain another one. Thus, the set of bank states is defined as

Definition 7. Consider a multi-resource theory $R_{\text {multi }}$ satisfying the asymptotic equivalence property with respect to the monotones $E_{\mathcal{F}_{1}}$ and $E_{\mathcal{F}_{2}}$. The set of bank states of the theory is a subset of the state space $\mathcal{S}(\mathcal{H})$ defined as,

$$
\begin{aligned}
\mathcal{F}_{\text {bank }}=\{\rho \in \mathcal{S}(\mathcal{H}) \mid \forall \sigma \in \mathcal{S}(\mathcal{H}), & E_{\mathcal{F}_{1}}(\sigma)>E_{\mathcal{F}_{1}}(\rho) \text { or } \\
& E_{\mathcal{F}_{2}}(\sigma)>E_{\mathcal{F}_{2}}(\rho) \text { or } \\
& \left.E_{\mathcal{F}_{1}}(\sigma)=E_{\mathcal{F}_{1}}(\rho) \text { and } E_{\mathcal{F}_{2}}(\sigma)=E_{\mathcal{F}_{2}}(\rho)\right\} .
\end{aligned}
$$

Within the set $\mathcal{F}_{\text {bank }}$ we can find different subsets of bank states with a fixed value of $E_{\mathcal{F}_{1}}$ and $E_{\mathcal{F}_{2}}$. We define each of these subsets as

$$
\mathcal{F}_{\text {bank }}\left(\bar{E}_{\mathcal{F}_{1}}, \bar{E}_{\mathcal{F}_{2}}\right)=\left\{\rho \in \mathcal{F}_{\text {bank }} \mid E_{\mathcal{F}_{1}}(\rho)=\bar{E}_{\mathcal{F}_{1}} \text { and } E_{\mathcal{F}_{2}}(\rho)=\bar{E}_{\mathcal{F}_{2}}\right\} .
$$

Notice that Eq. (34) implies that no state can be found with smaller values of both monotones $E_{\mathcal{F}_{i}}$ 's. In this way, the agent is not able to transform the state of the bank in a way in which both resources are extracted from it and stored in the batteries. Instead, they always need to trade resources. The set of bank states $\mathcal{F}_{\text {bank }}$ can be visualised in the resource diagram of the theory, see Fig. 4 . This set is represented by a curve on the boundary of the state space, connecting the points associated with $\mathcal{F}_{1}$ to those associated with $\mathcal{F}_{2}$. In appendix $B$ we show that, under the current assumptions, this curve is always convex, and in the following we focus our attention to those segments where the curve is strictly convex.

The subsets $\mathcal{F}_{\text {bank }}\left(\bar{E}_{\mathcal{F}_{1}}, \bar{E}_{\mathcal{F}_{2}}\right)$ 's represent individual points in the resource diagram describing the multi-resource theory, and they obey many of the properties satisfied by the invariant sets $\mathcal{F}_{i}$ 's. Indeed, one can show that

- For all $n \in \mathbb{N}$, we have that each subset of bank states is such that

$$
\mathcal{F}_{\text {bank }}^{(n)}\left(\bar{E}_{\mathcal{F}_{1}}, \bar{E}_{\mathcal{F}_{2}}\right)=\mathcal{F}_{\text {bank }}^{\otimes n}\left(\bar{E}_{\mathcal{F}_{1}}, \bar{E}_{\mathcal{F}_{2}}\right),
$$

that is, these subsets satisfy property F5b. This equality is proved in Prop. 24 of appendix D.2.

- Every subset $\mathcal{F}_{\text {bank }}\left(\bar{E}_{\mathcal{F}_{1}}, \bar{E}_{\mathcal{F}_{2}}\right)$ is convex, property F2, as shown in Prop. 25 in appendix D.2.

- Every subset $\mathcal{F}_{\text {bank }}\left(\bar{E}_{\mathcal{F}_{1}}, \bar{E}_{\mathcal{F}_{2}}\right)$, and its extensions to the many-copy case, is invariant under the class of allowed operations $\mathcal{C}_{\text {multi }}$ of the multi-resource theory, as shown in Lem. 26 in appendix D.2.

The second essential property for a bank is that the exchange rate needs only to depend on which state of the bank we choose to use. In our framework, it is the choice of the values $\bar{E}_{\mathcal{F}_{1}}$ and $\bar{E}_{\mathcal{F}_{2}}$, defining the subset $\mathcal{F}_{\text {bank }}\left(\bar{E}_{\mathcal{F}_{1}}, \bar{E}_{\mathcal{F}_{2}}\right)$, that determines the exchange rate at which the resources are converted. In order to obtain this exchange rate we introduce the following function, which quantifies how much the properties of the bank change during a transformation, and generalises the Helmholtz free energy used in thermodynamics. Given the subset of bank states $\mathcal{F}_{\text {bank }}\left(\bar{E}_{\mathcal{F}_{1}}, \bar{E}_{\mathcal{F}_{2}}\right)$, this function is defined as

$$
f_{\text {bank }}^{\bar{E}_{\mathcal{F}_{1}}, \bar{E}_{\mathcal{F}_{2}}}(\rho):=\alpha E_{\mathcal{F}_{1}}(\rho)+\beta E_{\mathcal{F}_{2}}(\rho)-\gamma,
$$

where $\alpha, \beta$, and $\gamma$ are non-negative constant factors, which depend on the subset of bank states we have chosen. In order to define the linear coefficients, we impose the following two properties for this function, 


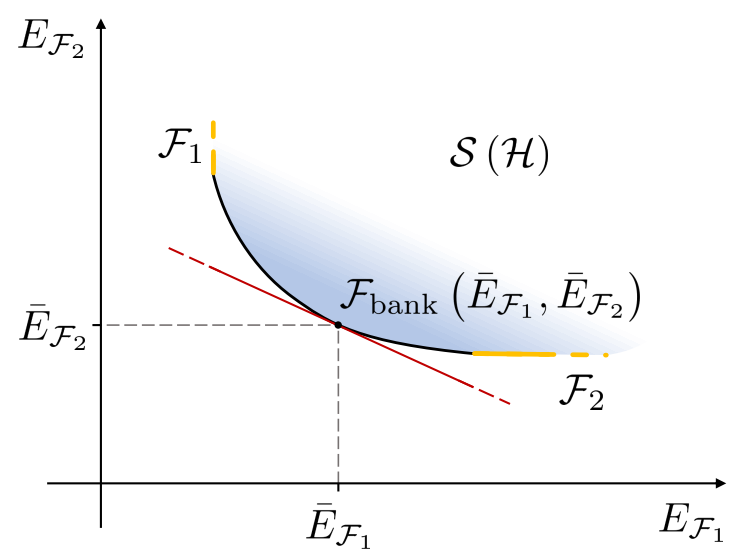

Figure 4: The set of bank states introduced in Eq. (34) is represented in the $E_{\mathcal{F}_{1}}-E_{\mathcal{F}_{2}}$ diagram. Only part of the state-space $\mathcal{S}(\mathcal{H})$ is shown, highlighted by the blue gradient region, together with the invariant sets of the theory $\mathcal{F}_{1}$ and $\mathcal{F}_{2}$, the two yellow segments. The black curve connecting these segments is the set of all the bank states of the theory $\mathcal{F}_{\text {bank. }}$. It is worth noting that this set does not include any states contained in the invariant sets $\mathcal{F}_{1}$ and $\mathcal{F}_{2}$. Indeed, a bank state needs to have a non-zero value of both resources in order to allow for general resource interconversion, and the states in $\mathcal{F}_{1}$ and $\mathcal{F}_{2}$ do not contain any amount of their associated resource. A specific subset of bank states, labelled by $\mathcal{F}_{\text {bank }}\left(\bar{E}_{\mathcal{F}_{1}}, \bar{E}_{\mathcal{F}_{2}}\right)$, is shown on the curve, see Eq. (35). Notice that, graphically, a bank state is one for which there exists no other state in the region immediately below and left. The red line, which is tangent to the set of bank states and passes through the

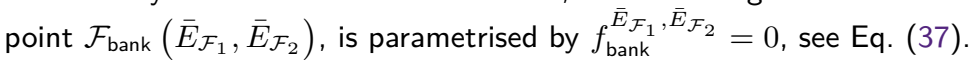

B1 The function $f_{\text {bank }}^{\bar{E}_{\mathcal{F}_{1}}, \bar{E}_{\mathcal{F}_{2}}}$ is equal to zero over the subset $\mathcal{F}_{\text {bank }}\left(\bar{E}_{\mathcal{F}_{1}}, \bar{E}_{\mathcal{F}_{2}}\right)$.

B2 The value of this function on the states contained in the subset $\mathcal{F}_{\text {bank }}\left(\bar{E}_{\mathcal{F}_{1}}, \bar{E}_{\mathcal{F}_{2}}\right)$ is minimum.

Notice that property B1 is there to set the zero of the function, and implies that

$$
\gamma=\alpha \bar{E}_{\mathcal{F}_{1}}+\beta \bar{E}_{\mathcal{F}_{2}}
$$

Property B2, instead, fixes the ratio between the constants $\alpha$ and $\beta$. This condition can be visualised in the resource diagram, and is equivalent to the request that, in such a diagram, the bank monotone is tangent to the state space, so that

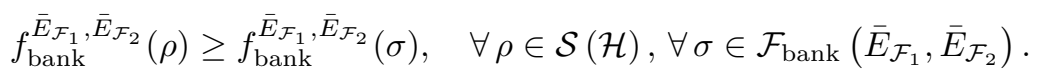

The above property is always satisfied under our working assumptions, since the curve of bank states is convex, see Fig. 4. We refer to this function as the bank monotone.

The bank monotone can be easily extended to the state space of $n$ copies of the system. The main difference is that, when we consider states in $\mathcal{S}\left(\mathcal{H}^{\otimes n}\right)$, the coefficient $\gamma$ is proportional to the number of copies $n$, and we write $\gamma=n\left(\alpha \bar{E}_{\mathcal{F}_{1}}+\beta \bar{E}_{\mathcal{F}_{2}}\right)$. This follows from property B1, together with the fact that the subset $\mathcal{F}_{\text {bank }}\left(\bar{E}_{\mathcal{F}_{1}}, \bar{E}_{\mathcal{F}_{2}}\right)$ satisfies property F5b, see Eq. (36). Since the function in Eq. (37) is a linear combination of the monotones $E_{\mathcal{F}_{1}}$ and $E_{\mathcal{F}_{2}}$, it is easy to show (see also appendix D.2) that it satisfies the properties listed in the following proposition

Proposition 8. Consider a resource theory $R_{\text {multi }}$ with allowed operations $\mathcal{C}_{\text {multi, satisfying asymptotic }}$ equivalence with respect to the monotones $E_{\mathcal{F}_{1}}$ and $E_{\mathcal{F}_{2}}$, i.e. the relative entropy distances from the invariant sets of the theory. Suppose that these sets satisfy the properties F1, F2, F3, and F5b. Then,

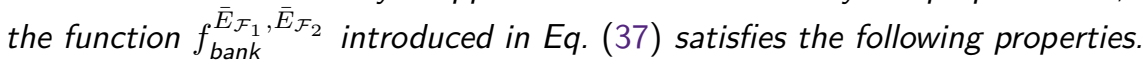

B3 The function $f_{\text {bank }}^{\bar{E}_{\mathcal{F}_{1}}, \bar{E}_{\mathcal{F}_{2}}}$ is additive.

B4 The function $f_{\text {bank }}^{\bar{E}_{\mathcal{F}_{1}}, \bar{E}_{\mathcal{F}_{2}}}$ is monotonic under partial tracing. 
B5 The function $f_{\text {bank }}^{\bar{E}_{\mathcal{F}_{1}}, \bar{E}_{\mathcal{F}_{2}}}$ is sub-extensive, i.e., this function scales at most linearly in the number of systems considered. More precisely, for any sequence of states $\left\{\rho_{n} \in \mathcal{S}\left(\mathcal{H}^{\otimes n}\right)\right\}$, we have that $f_{\text {bank }}^{\bar{E}_{\mathcal{F}_{1}}, \bar{E}_{\mathcal{F}_{2}}}\left(\rho_{n}\right)=O(n)$

B6 The function $f_{\text {bank }}^{\bar{E}_{\mathcal{F}_{1}}, \bar{E}_{\mathcal{F}_{2}}}$ is asymptotic continuous.

B7 The function $f_{\text {bank }}^{\bar{E}_{\mathcal{F}_{1}}, \bar{E}_{\mathcal{F}_{2}}}$ is monotonic under the set of allowed operations $\mathcal{C}_{\text {multi }}$, since $\alpha$ and $\beta$ are non-negative.

The third and last property we demand from a bank concerns the back-reaction it experiences during interconversion of resources. We want that, after the transformation, the state of the bank only changes infinitesimally with respect to the bank monotone associated with it. If this is the case, we can show that the exchange rate only changes infinitesimally, and therefore we can keep using the bank to inter-convert between resources at the same exchange rate. More concretely, we now consider a tripartite system composed by a bank $S$ and and two batteries, $B_{1}$ and $B_{2}$. Each of these subsystems is composed by many copies of the same fundamental system described by $\mathcal{H}$, for which we defined the notion of bank states. Thus, the bank $S$ is described by $\mathcal{H}_{S}=\mathcal{H}^{\otimes n}$, with $n \in \mathbb{N}$, and its initial state is given by $n$ copies of the bank state $\rho \in \mathcal{F}_{\text {bank }}\left(\bar{E}_{\mathcal{F}_{1}}, \bar{E}_{\mathcal{F}_{2}}\right)$. The batteries are described by $\mathcal{H}_{B_{i}}=\mathcal{H}^{\otimes m_{i}}, m_{i} \in \mathbb{N}$, where $i=1,2$. The states describing the batteries are $\omega_{1} \in \mathcal{S}\left(\mathcal{H}_{B_{1}}\right)$, and $\omega_{2} \in \mathcal{S}\left(\mathcal{H}_{B_{2}}\right)$, respectively.

A resource interconversion is an asymptotically reversible transformation

$$
\rho^{\otimes n} \otimes \omega_{1} \otimes \omega_{2} \stackrel{\text { asympt }}{\longleftrightarrow} \tilde{\rho}^{\otimes n} \otimes \omega_{1}^{\prime} \otimes \omega_{2}^{\prime},
$$

where $\tilde{\rho} \in \mathcal{S}(\mathcal{H}), \omega_{1}^{\prime} \in \mathcal{S}\left(\mathcal{H}_{B_{1}}\right)$, and $\omega_{2}^{\prime} \in \mathcal{S}\left(\mathcal{H}_{B_{2}}\right)$, satisfying the following property, see also Fig. 5,

$\mathbf{X} \mathbf{1}$ The state of the bank changes infinitesimally during the resource interconversion.

If $\rho \in \mathcal{F}_{\text {bank }}\left(\bar{E}_{\mathcal{F}_{1}}, \bar{E}_{\mathcal{F}_{2}}\right) \subset \mathcal{S}(\mathcal{H})$, then the state $\tilde{\rho} \in \mathcal{S}(\mathcal{H})$ is such that

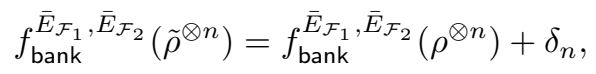

where $\delta_{n}>0$ is such that $\delta_{n} \rightarrow 0$ as $n \rightarrow \infty$.

It is worth noting that, according to the above definition, the bank is here acting as a catalyst, allowing for resource interconversion. Catalysts are used in resource theories to allow for state transformations which are otherwise impossible [37, 54]. These systems are generally described by resourceful states, and therefore are subject to strict constraints, for example the requirement that their initial state needs to be perfectly (or approximately) re-obtained at the end of the transformation [69]. These constraints are required since, otherwise, one might act on the catalyst and extract resources from it, thus trivializing the theory [70]. It is interesting to notice that our bank is similarly constrained, specifically by Eq. 41. As we see in the following theorem, this constrain is enough to allow for resource interconversion, but also to ensure a non-trivial behaviour of the theory (no resource is extracted for free).

We are now ready to introduce the interconversion relation which links the different amounts of resources exchanged, weighted by the exchange rate given by the bank. The theorem is proved in appendix D.1.

Theorem 9. Consider a resource theory $R_{\text {multi }}$ with two resources, equipped with the batteries $B_{1}$ and $B_{2}$. Suppose the theory satisfies asymptotic equivalence with respect to the monotones $E_{\mathcal{F}_{1}}$ and $E_{\mathcal{F}_{2}}$, i.e. the relative entropy distances from the invariant sets of the theory, and that these sets satisfy the properties F1, F2, F3, and F5b. Then, the resource interconversion of Eq. (40), where the bank has to transform in accord to condition $\mathrm{X} 1$, is solely regulated by the following relation,

$$
\alpha \Delta W_{1}=-\beta \Delta W_{2}+\delta_{n} .
$$

Furthermore, when the number of copies of the bank system $n$ is sent to infinity, we have that the above equation reduces to the following one, which we refer to as the interconversion relation,

$$
\Delta W_{1}=-\frac{\beta}{\alpha} \Delta W_{2},
$$

where the amount of resources exchanged $\Delta W_{i}$ is non-zero. 


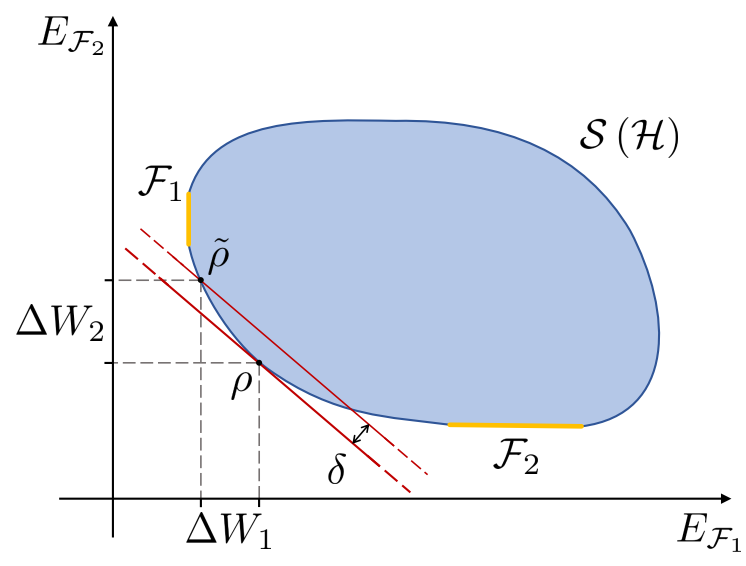

Figure 5: The state-space of the theory $\mathrm{R}_{\text {multi }}$ is represented in the $E_{\mathcal{F}_{1}}-E_{\mathcal{F}_{2}}$ diagram. The invariant sets of the theory, $\mathcal{F}_{1}$ and $\mathcal{F}_{2}$, are represented by the two yellow segments. The set of bank states $\mathcal{F}_{\text {bank }}$ lies on the boundary of the state-space, and is represented by the curve connecting the two invariant sets, see appendix $B$. The subset of bank states $\mathcal{F}_{\text {bank }}\left(\bar{E}_{\mathcal{F}_{1}}, \bar{E}_{\mathcal{F}_{2}}\right)$, where $\rho$ is contained, is represented by a point in the diagram. The red line which is tangent to the state-space and passes by the point associated to $\rho$ represents the set of states with value of the monotone $f_{\text {bank }}^{\bar{E}_{\mathcal{F}_{1}}, \bar{E}_{\mathcal{F}_{2}}}$ equal to 0 . The other line is given by all those states with a value $\delta>0$ of this monotone. We see that, by mapping $\rho$ into $\tilde{\rho}$, we can extract an amount $\Delta W_{1}$ of the first resource, while paying an amount $\Delta W_{2}$ of the second resource. Furthermore, one can show that when $\delta \rightarrow 0$, these two quantities tend to 0 as $\delta^{\frac{1}{2}}$, i.e., with a slower rate. It is then possible to keep the $\Delta W_{i}$ 's finite if we take $n \propto \delta^{-1}$ copies of the bank states, see the proof of Thm. 9, in appendix D.1. Thus, in the limit $n \rightarrow \infty$, the overall back-action on bank states associated with the conversion of resources can be made arbitrarily small.

Let us highlight some properties that a bank state needs to satisfy in order to allow for interconversion of resources from one battery to another, and vice versa. We show that to interconvert between the resources in both directions we need a bank state containing a non-zero amount of both resources. First notice that, since both parameters $\alpha$ and $\beta$ are non-negative, whenever we exchange between resources, we increase the amount contained in one of the batteries (for example, $\Delta W_{1}>0$ ) while decreasing the amount contained in the other $\left(\Delta W_{2}<0\right)$. However, the change in these two resources also depends on the transformation of the bank state, see Eq. (24). Therefore, one has to consider the bank state used for interconversion, and the amount of resources contained in it. When the bank state $\rho$ is such that $E_{\mathcal{F}_{1}}(\rho)>0$ and $E_{\mathcal{F}_{2}}(\rho)>0$, then interconversion can be achieved (in both directions) between $\Delta W_{1}$ and $\Delta W_{2}$, at the rate specified by Eq. (43). Moreover, as far as the amount of resources in the bank is non-zero, we can exchange any amount of one resource for the other (since we can take the number of copies of the bank to be infinite). This is the case of thermodynamics, where thermal states indeed contain a positive amount of both energy and entropy, the two resources of the theory, and Eq. (43) gives the conversion rate for Landauer's erasure.

Finally, let us consider what would happen if we were to allow the states in $\mathcal{F}_{1}$ or $\mathcal{F}_{2}$ (or in their intersection) to describe the bank. If the bank state were such that $E_{\mathcal{F}_{1}}(\rho)>0$ and $E_{\mathcal{F}_{2}}(\rho)=0$ (or vice versa), then we could only exchange in one direction, since we could gain the first resource while paying the second resource (or vice versa). If the bank state did not contain any amount of resources, $E_{\mathcal{F}_{1}}(\rho)=0$ and $E_{\mathcal{F}_{2}}(\rho)=0$, then we could not perform interconversion at all, because we would have to reduce the amount of one of them within the bank. However, this would not be possible since the amount of resource stored in a (bank) state cannot be negative. As a result, the multi-resource theories in which an interesting interconversion relation can be found are the ones in which the invariant sets of the theory do not intercept, see the right panel of Fig. 2.

\subsection{Bank monotones and the relative entropy distance}

We start this section with an example concerning different models to describe thermodynamics, and the connection between these models. In the last part of Sec. 2.2, we have introduced a multi-resource theory whose resources are energy and entropy (or, information). For this theory, the bank states are thermal states at a given temperature $T$. We can move from this description of thermodynamics to a 
different one, based on a single-resource theory, by enlarging the class of operations in such a way that the agent can freely add ancillary systems in a thermal state with temperature $T$. This corresponds to the physical situation in which the system is put in contact with an infinite thermal bath. The singleresource theory we obtain is analogous to the one of Thermal Operations [10,11], and its resource quantifier is unique. In fact, we can show that the bank monotone of the multi-resource theory and the resource quantifier of the single resource theory both coincides (modulo a multiplicative factor) with $F-F_{\beta}$, where $F$ is the Helmholtz free energy of the state whose resource we are quantifying, and $F_{\beta}$ is the Helmholtz free energy of the thermal state with temperature $T=\beta^{-1}$.

In the following we study the connection between a general multi-resource theory and the singleresource theory obtained by enlarging the allowed operations with the possibility of adding ancillary systems described by bank states in $\mathcal{F}_{\text {bank }}\left(\bar{E}_{\mathcal{F}_{1}}, \bar{E}_{\mathcal{F}_{2}}\right)$. We find that the bank monotone of Eq. (37), $f_{\text {bank }}^{\bar{E}_{\mathcal{F}_{1}}, \bar{E}_{\mathcal{F}_{2}}}$, coincides with the unique measure of resource for the obtained single-resource theory. As a result, we find that property $\mathrm{X} 1$, which regulates the exchange of resources in the multi-resource theory, can be understood as the condition that the resource characterising the bank does not increase during the transformation. Furthermore, we show that, when the subset of bank states $\mathcal{F}_{\text {bank }}\left(\bar{E}_{\mathcal{F}_{1}}, \bar{E}_{\mathcal{F}_{2}}\right)$ contains a full-rank state, the monotone $f_{\text {bank }}^{\bar{E}_{\mathcal{F}_{1}}, \bar{E}_{\mathcal{F}_{2}}}$ is proportional to the relative entropy distance from this subset. Let us now introduce the single-resource theory which can be derived from $\mathrm{R}_{\text {multi }}$ by allowing the possibility of adding ancillary systems described by specific bank states.

Definition 10. Consider the two-resource theory $R_{\text {multi }}$ with allowed operations $\mathcal{C}_{\text {multi }}$ and invariant sets $\mathcal{F}_{1}$ and $\mathcal{F}_{2}$ which satisfy the properties F1, F2, F3, and F5b. Consider the bank set $\mathcal{F}_{\text {bank }}\left(\bar{E}_{\mathcal{F}_{1}}, \bar{E}_{\mathcal{F}_{2}}\right)$ introduced in Eq. (35). We define the single-resource theory $R_{\text {single }}$ as that theory whose class of allowed operations $\mathcal{C}_{\text {single }}$ is composed by the following three fundamental operations,

1. Add an ancillary system described by $n \in \mathbb{N}$ copies of a bank state $\rho_{P} \in \mathcal{F}_{\text {bank }}\left(\bar{E}_{\mathcal{F}_{1}}, \bar{E}_{\mathcal{F}_{2}}\right)$.

2. Apply any operation $\mathcal{E} \in \mathcal{C}_{\text {multi }}$ to system and ancilla.

3. Trace out the ancillary systems.

The most general operation in $\mathcal{C}_{\text {single }}$ which does not change the number of systems between its input and output is

$$
\mathcal{E}^{(s)}(\rho)=\operatorname{Tr}_{P(n)}\left[\mathcal{E}\left(\rho \otimes \rho_{P}^{\otimes n}\right)\right],
$$

where we are partial tracing over the degrees of freedom $P^{(n)}$, that is, over the ancillary system initially in $\rho_{P}^{\otimes n}$.

The bank monotone associated with the bank set $\mathcal{F}_{\text {bank }}\left(\bar{E}_{\mathcal{F}_{1}}, \bar{E}_{\mathcal{F}_{2}}\right)$, see Eq. (37), is the unique quantifier for the single-resource theory $\mathrm{R}_{\text {single. }}$ In order to show the uniqueness of this monotone, we first have to show that the single-resource theory satisfies asymptotic equivalence.

Theorem 11. Consider the two-resource theory $R_{\text {multi }}$ with allowed operations $\mathcal{C}_{\text {multi, }}$ and invariant sets $\mathcal{F}_{1}$ and $\mathcal{F}_{2}$ which satisfy the properties F1, F2, F3, and F5b. Suppose the theory satisfies the asymptotic equivalence property with respect to the monotones $E_{\mathcal{F}_{1}}$ and $E_{\mathcal{F}_{2}}$. Then, given the subset of bank states $\mathcal{F}_{\text {bank }}\left(\bar{E}_{\mathcal{F}_{1}}, \bar{E}_{\mathcal{F}_{2}}\right)$, the single-resource theory $R_{\text {single }}$ with allowed operations $\mathcal{C}_{\text {single }}$ satisfies the

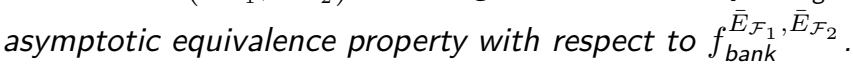

The proof of this theorem can be found in appendix D.1, and we provide a geometric sketch of it in Fig. 6. As a side remark, notice that the functions $E_{\mathcal{F}_{1}}$ and $E_{\mathcal{F}_{2}}$ are not monotonic under the set of allowed operations $\mathcal{C}_{\text {single. }}$. This follows from the fact that we can now replace any state of the system with a state in $\mathcal{F}_{\text {bank }}\left(\bar{E}_{\mathcal{F}_{1}}, \bar{E}_{\mathcal{F}_{2}}\right)$, since we are free to add an ancillary system in such state, and to perform a swap between main system and ancilla (since this operation belongs to $\mathcal{C}_{\text {multi }}$ ). Then, if the bank state contains a non-zero amount of resources, meaning that $\bar{E}_{\mathcal{F}_{i}}>0$ for $i=1$, 2, we can always find a state in $\mathcal{S}(\mathcal{H})$ with lower value of either $E_{\mathcal{F}_{1}}$ or $E_{\mathcal{F}_{2}}$ (but not both at the same time), and therefore the above transformation would increase the value of this monotone.

From the above theorem it follows an interesting link between the bank monotone $f_{\text {bank }}^{\bar{E}_{\mathcal{F}_{1}}, \bar{E}_{\mathcal{F}_{2}}}$, defined in Eq. (37), and the relative entropy distance from the set of bank states $\mathcal{F}_{\text {bank }}\left(\bar{E}_{\mathcal{F}_{1}}, \bar{E}_{\mathcal{F}_{2}}\right)$. Indeed, when this set of states contains at least one full-rank state, we can prove that these two functions 

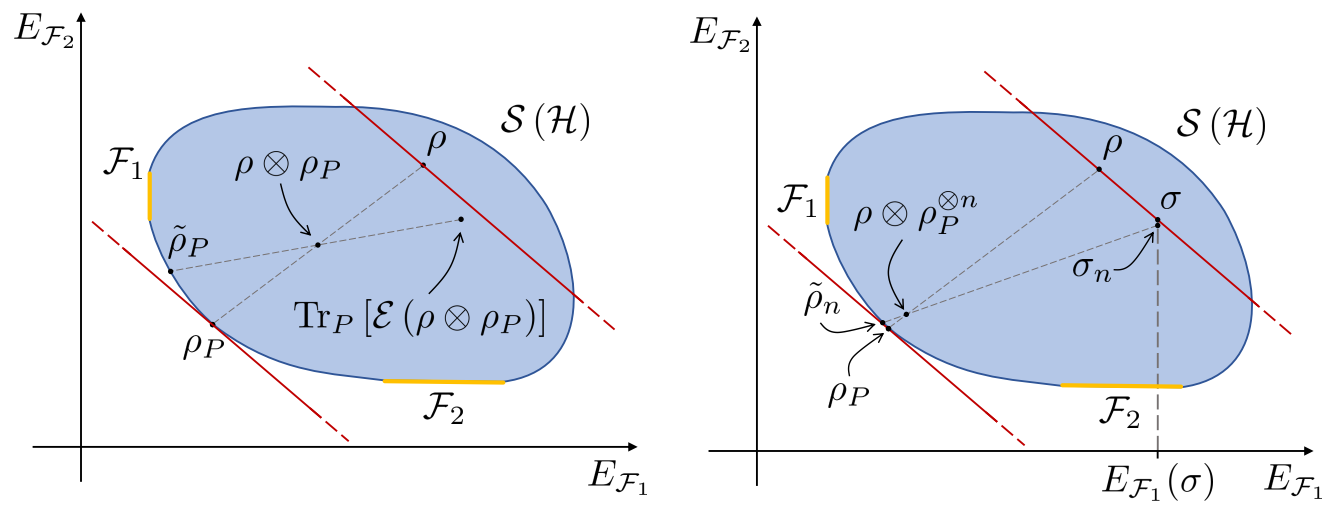

Figure 6: We sketch a geometric proof of Thm. 11 using the $E_{\mathcal{F}_{1}}-E_{\mathcal{F}_{2}}$ diagram. The blue region is the statespace $\mathcal{S}(\mathcal{H})$, the yellow segments are the invariant sets $\mathcal{F}_{1}$ and $\mathcal{F}_{2}$, and the red lines highlight the states with same value of monotone $f_{\text {bank }} \bar{E}_{\mathcal{F}_{1}}, \bar{E}_{\mathcal{F}_{2}}$. Notice that in this figure we are using the fact that, when $\mathcal{F}_{i}$ satisfies property F5b, the monotone $E_{\mathcal{F}_{i}}$ is such that $E_{\mathcal{F}_{i}}(\rho \otimes \sigma)=E_{\mathcal{F}_{i}}(\rho)+E_{\mathcal{F}_{i}}(\sigma)$ for any two states $\rho$ and $\sigma$ in $\mathcal{S}(\mathcal{H})$, see Lem. 23. To represent the state $\rho \otimes \sigma$ in the diagram, we renormalise its values of the $E_{\mathcal{F}_{i}}$ 's by dividing them by the number of copies considered, in this case by two. Left. We first sketch why the function $f_{\text {bank }}^{\bar{E}_{\mathcal{F}_{1}}, \bar{E}_{\mathcal{F}_{2}}}$ is monotonic under the set of allowed operations $\mathcal{C}_{\text {single. }}$. Consider a system initially described by the state $\rho$, and add to it an ancillary system described by the bank state $\rho_{P} \in \mathcal{F}_{\text {bank }}\left(\bar{E}_{\mathcal{F}_{1}}, \bar{E}_{\mathcal{F}_{2}}\right)$. The global system is then represented by a point in the middle of the segment connecting $\rho$ and $\rho_{P}$. We can transform the global state with the help of a sub-linear ancilla and of the operation $\mathcal{E} \in \mathcal{C}_{\text {multi }}$, mapping it into the state $\sigma \otimes \tilde{\rho}_{P}$ with same value of $E_{\mathcal{F}_{1}}$ and $E_{\mathcal{F}_{2}}$. If we take $\tilde{\rho}_{P}$ to be on the boundary of the state-space, we can easily see

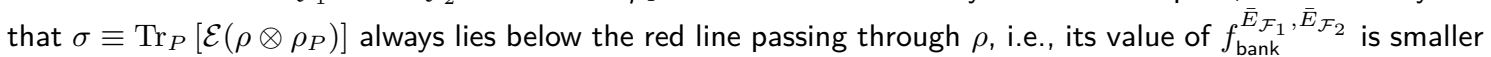
than the one for $\rho$. Right. We now sketch how to map between states with the same value of the monotone

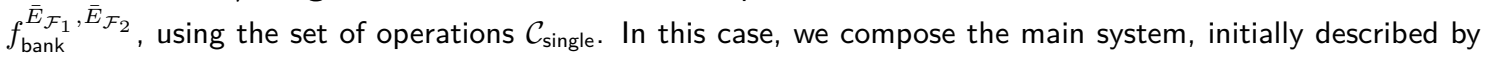
$\rho$, with an additional one described by $n$ copies of $\rho_{P}$. We then use an operation $\mathcal{E} \in \mathcal{C}_{\text {multi }}$, together with a sub-linear ancilla, and we ask the final state of the system, $\sigma_{n}=\operatorname{Tr}_{P^{(n)}}\left[\mathcal{E}\left(\rho \otimes \rho_{P}^{\otimes n}\right)\right]$ to have the same value of $E_{\mathcal{F}_{1}}$ of the target state $\sigma$. It is then easy to show that, as $n \rightarrow \infty$, the state $\sigma_{n}$ tends to $\sigma$, while the $n$ copies of the final state of the ancilla, $\tilde{\rho}_{n}$, tends to the bank state $\rho_{P}$.

have to coincide, modulo a multiplicative factor. This is a consequence of the fact that $R_{\text {single }}$ satisfies asymptotic equivalence, which implies the uniqueness of the resource measure, and of the fact that both the bank monotone and the relative entropy distance from the bank set satisfy the same properties, in particular monotonicity under the operations in $\mathcal{C}_{\text {single }}$ and asymptotic continuity. We can express this fact in the following corollary, whose proof can be found in appendix D.1.

Corollary 12. Consider the two-resource theory $R_{\text {multi }}$ with allowed operations $\mathcal{C}_{\text {multi, and invariant }}$ sets $\mathcal{F}_{1}$ and $\mathcal{F}_{2}$ which satisfy the properties F1, F2, F3, and F5b. Suppose the theory satisfies the asymptotic equivalence property with respect to the monotones $E_{\mathcal{F}_{1}}$ and $E_{\mathcal{F}_{2}}$. If the subset of bank states $\mathcal{F}_{\text {bank }}\left(\bar{E}_{\mathcal{F}_{1}}, \bar{E}_{\mathcal{F}_{2}}\right)$ contains a full-rank state, then the bank monotone $f_{\text {bank }}^{\bar{E}_{\mathcal{F}_{1}}, \bar{E}_{\mathcal{F}_{2}}}$ coincides with the relative entropy distance from this subset of states, modulo a multiplicative constant.

We close the section with the remark that, in the currently known scenarios, the bank subsets always contain at least a full-rank state, and in fact we find that, for these theories, the above correspondence between the bank monotone of Eq. (37) and the relative entropy distance is satisfied. An example is the multi-resource theory of thermodynamics, in which the relative entropy distance from a thermal state at a given temperature is indeed equal to the linear combination of the average energy and the entropy of a system. Other examples can be found in Sec. 5.

\subsection{First law for multi-resource theories}

We can now introduce a general first law for multi-resource theories with disjoint invariant sets, see the right panel of Fig. 2. In order for this law to be valid, we need access to the batteries, the bank, and the main system. Within this setting, the first law consists of a single relation which links the different amount of resources exchanged with the batteries, the $\Delta W_{i}$ 's, with the change in bank monotone over 
the state of the main system. The idea is that, contrary to what seen in Sec. 3.2, a state transformation over the main system is possible, when a bank is present, if this single relation is satisfied. Indeed, we do not need to use a fixed amount of each resource, since they are inter-convertible using the bank system.

In more detail, we consider a theory $\mathrm{R}_{\text {multi }}$ that, for simplicity, has just two resources. The invariant sets are $\mathcal{F}_{1}$ and $\mathcal{F}_{2}$, they satisfy the properties $F 1, F 2, F 3$ and $F 5 b$, and the theory satisfies the asymptotic equivalence property with respect to the monotones $E_{\mathcal{F}_{1}}$ and $E_{\mathcal{F}_{2}}$. The global system is divided into four partitions, the main system $S$, the bank $P$, and the batteries $B_{1}$ and $B_{2}$. We assume the bank to be initially described by a state $\rho_{P} \in \mathcal{F}_{\text {bank }}\left(\bar{E}_{\mathcal{F}_{1}}, \bar{E}_{\mathcal{F}_{2}}\right)$, where this subset contains at least one full-rank state. The relevant monotone for the interconversion of resources is then the relative entropy distance from the subset $\mathcal{F}_{\text {bank }}\left(\bar{E}_{\mathcal{F}_{1}}, \bar{E}_{\mathcal{F}_{2}}\right)$, as shown in Cor. 12.

Suppose that the main system is initially described by the state $\rho \in \mathcal{S}\left(\mathcal{H}_{S}\right)$, and we want to map it into the state $\sigma \in \mathcal{S}\left(\mathcal{H}_{S}\right)$, with possibly a different value of $E_{\mathcal{F}_{1}}$ and $E_{\mathcal{F}_{2}}$. If we do not have access to the bank, then the amount of resources we need to exchange is given by the difference of the monotones $E_{\mathcal{F}_{i}}$ 's between the initial and final state of the main system, see Eq. (28) in Sec. 3.3. But since we have access to the battery, we can exchange between the resources, and we are not obliged any more to provide a fixed amount for each resource. In order to show this, consider the global initial state $\rho \otimes \rho_{P} \otimes \omega_{1} \otimes \omega_{2}$, describing the main system, the bank, and the two batteries $B_{1}$ and $B_{2}$. Then, we (asymptotically) map this global state, using the allowed operations $\mathcal{C}_{\text {multi }}$, into the final state $\sigma \otimes \tilde{\rho}_{P} \otimes \omega_{1}^{\prime} \otimes \omega_{2}^{\prime}$, where the final state of the bank is $\tilde{\rho}_{P}$, and the batteries $B_{1}$ and $B_{2}$ have final state $\omega_{1}^{\prime}$ and $\omega_{2}^{\prime}$, respectively. Due to asymptotic equivalence, this state transformation is possible only if the monotones $E_{\mathcal{F}_{i}}$ 's are preserved. However, the final state of the bank only has to satisfy property $\mathrm{X} 1$, and we have shown in Sec. 4.1 that such constraint still allows us to exchange an arbitrary amount of resources, see Thm. 9. As a result, there is a single relation that regulates the state transformation over the main system,

Corollary 13. Consider the two-resource theory $R_{\text {multi }}$ with allowed operations $\mathcal{C}_{\text {multi, }}$ and invariant sets $\mathcal{F}_{1}$ and $\mathcal{F}_{2}$ which satisfy the properties F1, F2, F3, and F5b. Suppose the theory satisfies the asymptotic equivalence property with respect to the monotones $E_{\mathcal{F}_{1}}$ and $E_{\mathcal{F}_{2}}$, and that the global system is divided into a main system $S$, a bank described by the set of states $\mathcal{F}_{\text {bank }}\left(\bar{E}_{\mathcal{F}_{1}}, \bar{E}_{\mathcal{F}_{2}}\right)$ (which contains at least one full-rank state), and two batteries $B_{1}$ and $B_{2}$. Then, a transformation which maps the state of the main system from $\rho$ into $\sigma$, where these states are completely general, only has to satisfy the following relation

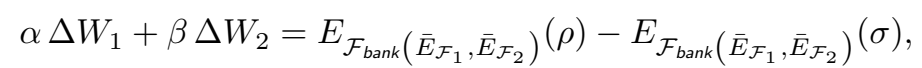

where each $\Delta W_{i}$ is defined as the difference in the monotone $E_{\mathcal{F}_{i}}$ over the final and initial state of the battery $B_{i}$, see Eq. (23), and $E_{\mathcal{F}_{\text {bank }}\left(\bar{E}_{\mathcal{F}_{1}}, \bar{E}_{\mathcal{F}_{2}}\right)}$ is the relative entropy distance from the set of states describing the bank.

We refer to Eq. (45) as the first law of multi-resource theories. Indeed, for the resource theory of thermodynamics, where energy and entropy are the two resources, and the bank is given by an infinite thermal reservoir with a given temperature $T$, this equation corresponds to the First Law of Thermodynamics. In fact, in the thermodynamic scenario we have that $\Delta W_{1}=-\Delta U$, where $U$ is the internal energy of the system, while $\Delta W_{2}=\Delta S$ is the change in entropy in the system. The change in relative entropy distance on the main system is proportional to the change in Helmholtz free-energy, which in turn is equal to the work extracted from the system, $W$. The linear coefficients in the equation can be computed from Eq. (37), knowing that the bank monotone is equal to the relative entropy distance from the thermal state with temperature $T$. It is easy to show that $\alpha=T^{-1}$ and $\beta=1$. If we re-arrange the equation, and we define $Q=T \Delta S$ as the amount of heat absorbed by the system, we obtain $\Delta U=Q-W$, that is, the First Law of Thermodynamics.

\section{Examples}

In this section we present two examples of multi-resource theories where an interconversion relation can be derived. The first one is thermodynamics for multiple conserved quantities (even non-commuting ones), while the second one concerns local control under energetic restrictions. In both examples we 


\section{Instructions}

1. Identify constraints and conservation laws of the theory.

2. Define allowed operations $\mathcal{C}_{\text {multi }}$ and identify invariant sets $\left\{\mathcal{F}_{i}\right\}$.

3. Use flowchart to identify the properties of monotones and invariant sets.

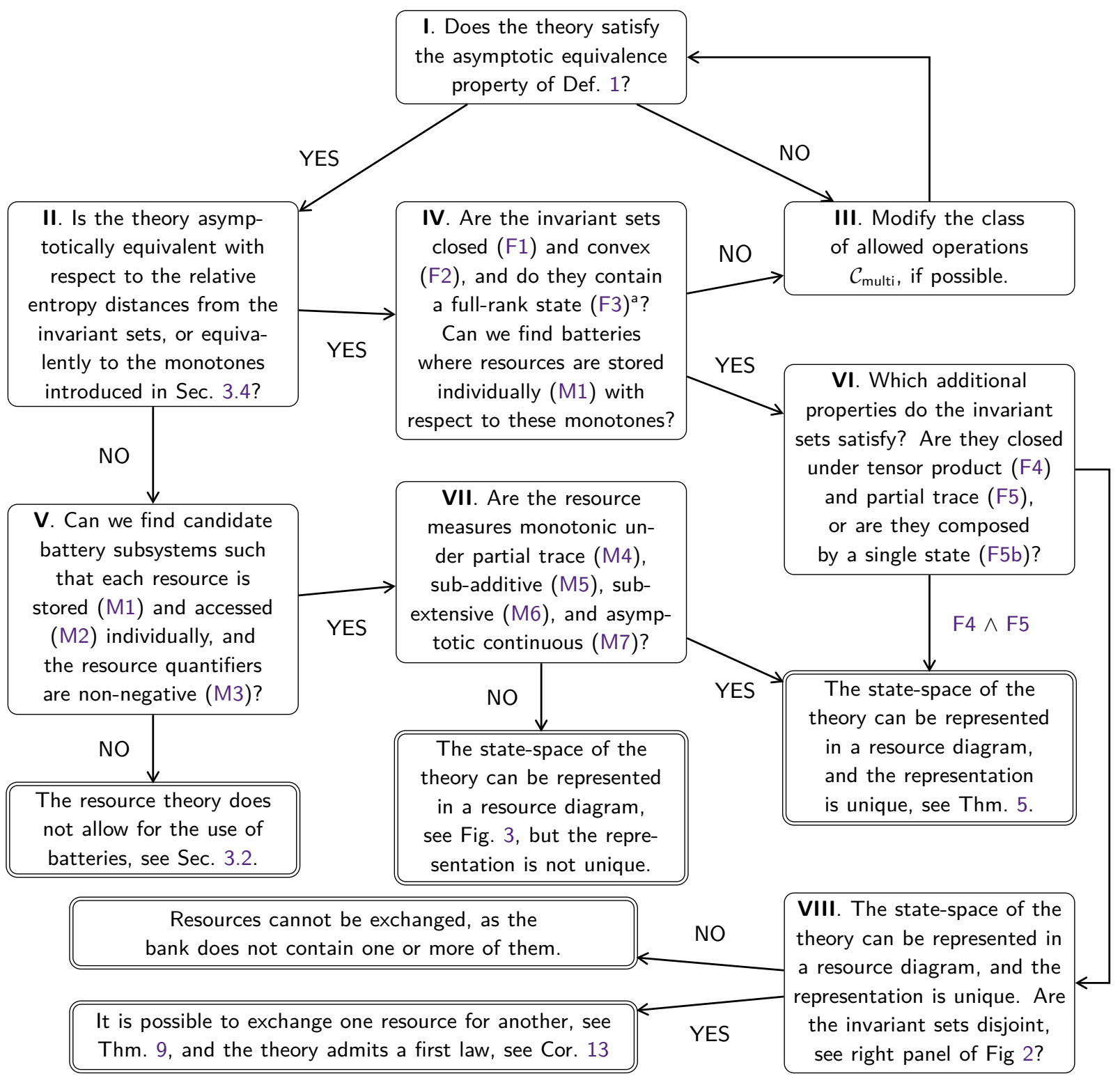

${ }^{\text {a }}$ For the monotones introduced in Sec. 3.4, property F3 is not relevant.

Figure 7: How to apply the results of this paper to an arbitrary resource theory.

describe the state-space (and we represent it with a resource diagram), we find the bank states of the theory, and we derive an interconversion relation for the different resources. Furthermore, in both cases we find that the bank monotone is proportional to the relative entropy distance from the given set of bank states, as expected from Cor. 12.

Before we introduce the examples, we provide a flowchart 7 that should help the reader in building a multi-resource theory. In particular, the flowchart clarifies in which situations each of the results we obtain hold for a specific theory. This tool should be used as follows, 
- The fundamental constraints and conservation laws of the task under consideration should be identified, and together with them the resources composing the theory.

- Given the set of resources for the theory, we define the class of allowed operations $\mathcal{C}_{\text {multi }}$ as in Eq. (11), and we identify the invariant sets of the theory $\left\{\mathcal{F}_{i}\right\}_{i=1}^{m}$.

- Checking whether asymptotic equivalence holds for the multi-resource theory is the first step of the flowchart (box I in Fig. 7). To show that the theory satisfies this property, we need to find a protocol which maps between states with same values of a given set of monotones.

- If the theory satisfies asymptotic equivalence, we can focus on the properties of the monotones and of the invariant sets. Following the flowchart, we can then easily identify which properties and features hold for the theory under consideration.

The flowchart here introduced is used in the first example to clarify how to characterise a multi-resource theory.

\subsection{Thermodynamics of multiple-conserved quantities}

In this example we consider the resource theory of thermodynamics in the presence of multiple conserved quantities (even in the case in which these quantities do not commute) $[48,49,51]$. Our system is a $d$-level quantum system, and for simplicity, we only consider two conserved quantities $A$ and $B$. The allowed operations are Thermal Operations $[10,11]$, composed by unitary operators which commute with both $A$ and $B$. This set of maps can be obtained as a proper subset of the intersection between the allowed operations of the following single-resource theories,

- The resource theory of the quantity $A$. The allowed operations are all the average- $A$-nonincreasing maps, whose invariant set is composed by a single state, $\mathcal{F}_{A}=\left\{\left|a_{0}\right\rangle\left\langle a_{0}\right|\right\}$, the eigenstate of $A$ associated with its minimum eigenvalue $a_{0}$ (for simplicity, we here assume it to be non-degenerate). From Sec. 3.4 it follows that this theory has a monotone of the form $M_{A}(\rho)=\operatorname{Tr}[A \rho]-a_{0}$.

- The resource theory of the quantity $B$. The allowed operations are all the average- $B$-nonincreasing maps, whose invariant set is composed by a single state, $\mathcal{F}_{B}=\left\{\left|b_{0}\right\rangle\left\langle b_{0}\right|\right\}$, the eigenstate of $B$ associated with its minimum eigenvalue $b_{0}$ (for simplicity, we here assume it to be non-degenerate). From Sec. 3.4 it follows that this theory has a monotone of the form $M_{B}(\rho)=\operatorname{Tr}[B \rho]-b_{0}$.

- The resource theory of purity, where the allowed operations are all the maps whose fix point is the maximally-mixed state $\mathcal{F}_{S}=\left\{\frac{\mathbb{I}}{d}\right\}$ (unital maps). One monotone of the theory is the relative entropy distance from $\frac{\mathbb{I}}{d}$, that is, $E_{\mathcal{F}_{S}}(\rho)=\log d-S(\rho)$ where $S(\cdot)$ is the von Neumann entropy.

We can now make use of the flowchart to characterise the multi-resource theory. Box $\mathbf{I}$ in the flowchart asks whether or not the considered multi-resource theory satisfies asymptotic equivalence. In Refs. $[35,36]$ it has been shown that, indeed, a resource theory of this kind does satisfy the asymptotic equivalence property of Def. 1 with respect to the monotones $M_{A}, M_{B}$ and $E_{\mathcal{F}_{S}}$. Furthermore, it is easy to see that these monotones are either relative entropy distances from the set of invariant states, or that they are of the form given in Eq. (31). This implies that we can answer positively to box II in the flowchart.

We now need to consider the properties of the invariant sets of the theory, which in turn determine the properties of the monotones. It is easy to show that these sets are closed (property F1) and convex (property F2). Furthermore, $\mathcal{F}_{S}$ contains a full-rank state (property F3), that implies asymptotic continuity of the associated monotone, see Refs. [63, 64]. The fact that the other sets do not contain a full-rank state is not problematic since we are considering monotones of the form of Eq. (31), that are nevertheless asymptotic continuous, see Prop. 22. Thus, the invariant sets satisfy all the properties required in box IV and we now need to construct batteries able to store the different resources separately (property M1).

For the first kind of resource, this can be achieved by selecting two pure states with different average values of $A$, and same average values of $B$. The battery $B_{A}$, storing the first kind of resource, 
is then composed by a certain number of copies of these two states, where the number varies when we extract/store the resource. A similar construction can be done for the other battery $B_{B}$. For the purity battery, we can take a system with degenerate $A$ and $B$, and take states with a certain number of copies of a pure state and mixed state. If this construction is possible, then we can answer positively to box IV in the flowchart.

We can now study the properties of the invariant sets, specifically their closure with respect to tensor product (property F4) and partial trace (property F5). Since each invariant set is composed by a single state, we find that both these properties and property F5b are satisfied. Thus, from box $\mathbf{V I}$ we can move to box VIII, and therefore the theory can be studied with a resource diagram, see Fig. 8 and the representation is unique.

Let us now consider a reversible transformation, described by the following equation

$$
\rho^{\otimes n} \otimes \omega_{A} \otimes \omega_{B} \otimes \omega_{S} \stackrel{\text { asympt }}{\longleftrightarrow} \sigma^{\otimes n} \otimes \omega_{A}^{\prime} \otimes \omega_{B}^{\prime} \otimes \omega_{S}^{\prime},
$$

where the $n$ copies of $\rho$ and $\rho^{\prime}$ describe the main system at the beginning and the end of the transformation, and the states $\omega_{i}$ and $\omega_{i}^{\prime}$ are the initial and final states of the battery $B_{i}$, for $i=A, B, S$. According to asymptotic equivalence, the transformation is possible if

$$
\begin{aligned}
\Delta W_{A} & =M_{A}^{\infty}(\rho)-M_{A}^{\infty}(\sigma)=\operatorname{Tr}[A(\rho-\sigma)], \\
\Delta W_{B} & =M_{B}^{\infty}(\rho)-M_{B}^{\infty}(\sigma)=\operatorname{Tr}[B(\rho-\sigma)], \\
\Delta W_{S} & =E_{\mathcal{F}_{S}}^{\infty}(\rho)-E_{\mathcal{F}_{S}}^{\infty}(\sigma)=S(\sigma)-S(\rho) .
\end{aligned}
$$

To answer the last box of the flowchart, box VIII, we need to focus on the resources contained in the bank states. Indeed, in order to get an interconversion relation and a first law we need the bank states to contain a non-zero amount of each resource. This has to be the case for the current resource theory, since the invariant sets do not intercept each other. Therefore, this theory admits a first law, as we are going to show. It can be easily shown, using Jaynes principle [67], that the bank states are of the following form

$$
\tau_{\beta_{1}, \beta_{2}}=\frac{e^{-\beta_{1} A-\beta_{2} B}}{Z},
$$

where the parameters $\beta_{1}, \beta_{2} \in[0, \infty)$, and $Z=\operatorname{Tr}\left[e^{-\beta_{1} A-\beta_{2} B}\right]$ is the partition function of the system. These states are known in thermodynamics as the grand-canonical ensemble. Each $\tau_{\beta_{1}, \beta_{2}}$ is a bank state with a different value of resource $A$, resource $B$, and purity. The value of these three resources only depends on the parameters $\beta_{1}$ and $\beta_{2}$. In order to find the interconversion relation we need to construct the bank monotone

$$
f_{\text {bank }}^{\bar{\beta}_{1}, \bar{\beta}_{2}}(\rho)=\alpha_{\bar{\beta}_{1}, \bar{\beta}_{2}} M_{A}(\rho)+\gamma_{\bar{\beta}_{1}, \bar{\beta}_{2}} M_{B}(\rho)+\delta_{\bar{\beta}_{1}, \bar{\beta}_{2}} E_{\mathcal{F}_{S}}(\rho)-\xi_{\bar{\beta}_{1}, \bar{\beta}_{2}}
$$

which is equal to zero over the bank state $\tau_{\bar{\beta}_{1}, \bar{\beta}_{2}}$. Properties B1 and B2 provide a geometrical way of building the monotone. If we represent the state space in a three-dimensional diagram (where the axes are given by $M_{A}, M_{B}$, and $\left.E_{\mathcal{F}_{S}}\right)$, then the hyperplane defined by the equation $f_{\text {bank }}^{\bar{\beta}_{1}, \bar{\beta}_{2}}=0$ is tangent to the state space and only intercepts it in $\tau_{\bar{\beta}_{1}, \bar{\beta}_{2}}$, see Fig. 8 for an example.

The hyperplane defined by $f_{\text {bank }}^{\bar{\beta}_{1}, \bar{\beta}_{2}}=0$ is identified by the normal vector

$$
\hat{n}=\hat{r}_{1} \times \hat{r}_{2}, \quad \text { where } \hat{r}_{i}=\left(\frac{\partial M_{A}\left(\tau_{\bar{\beta}_{1}, \bar{\beta}_{2}}\right)}{\partial \beta_{i}} ; \frac{\partial M_{B}\left(\tau_{\bar{\beta}_{1}, \bar{\beta}_{2}}\right)}{\partial \beta_{i}} ; \frac{\partial E_{\mathcal{F}_{S}}\left(\tau_{\bar{\beta}_{1}, \bar{\beta}_{2}}\right)}{\partial \beta_{i}}\right)^{T} \text { for } i=1,2 \text {. }
$$

The parametric equation of the hyperplane then gives us the expression of the monotone,

$$
f_{\text {bank }}^{\bar{\beta}_{1}, \bar{\beta}_{2}}(\rho)=n_{1}\left(M_{A}(\rho)-M_{A}\left(\tau_{\bar{\beta}_{1}, \bar{\beta}_{2}}\right)\right)+n_{2}\left(M_{B}(\rho)-M_{B}\left(\tau_{\bar{\beta}_{1}, \bar{\beta}_{2}}\right)\right)+n_{3}\left(E_{\mathcal{F}_{S}}(\rho)-E_{\mathcal{F}_{S}}\left(\tau_{\bar{\beta}_{1}, \bar{\beta}_{2}}\right)\right),
$$

where $n_{i}$ is the $i$-th component of the normal vector $\hat{n}$. By evaluating the monotones $M_{A}, M_{B}, E_{\mathcal{F}_{S}}$, and their derivatives we find that $f_{\text {bank }}^{\bar{\beta}_{1}, \bar{\beta}_{2}}$ is equal (modulo a positive multiplicative factor depending on the parameters $\bar{\beta}_{1}$ and $\bar{\beta}_{2}$ ) to the relative entropy distance from $\tau_{\bar{\beta}_{1}, \bar{\beta}_{2}}$,

$$
f_{\text {bank }}^{\bar{\beta}_{1}, \bar{\beta}_{2}}(\rho) \propto E_{\tau_{\bar{\beta}_{1}, \bar{\beta}_{2}}}(\rho)=\bar{\beta}_{1} \operatorname{Tr}[\rho A]+\bar{\beta}_{2} \operatorname{Tr}[\rho B]-S(\rho)+\log Z .
$$




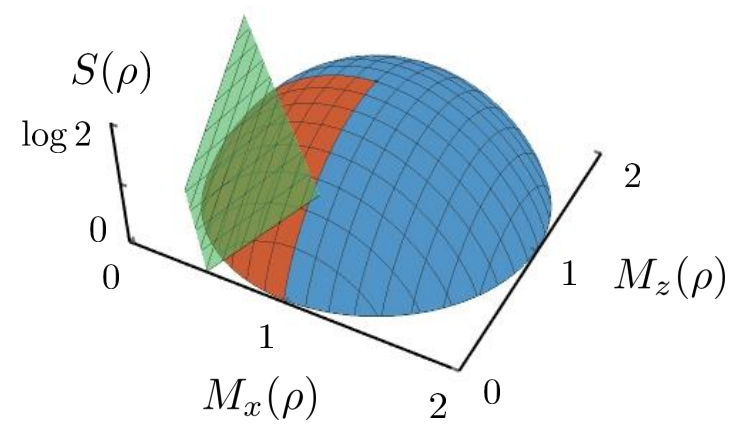

Figure 8: The state space of the multi-resource theory of thermodynamics and conserved angular momenta (along the $x$ and $z$ axes). On the surface we find the states $\tau_{\beta_{1}, \beta_{2}}$ defined in Eq. (50), where $\beta_{1}$ and $\beta_{2}$ take values in $\mathbb{R}$. The red surface is the set of bank states, with $\beta_{1}$ and $\beta_{2}$ are both non-negative, and the green plane is tangent to the state space in the point associated with $\tau_{\bar{\beta}_{1}, \bar{\beta}_{2}}$. The equation of the plane gives the monotone $f_{\text {bank }}^{\bar{\beta}_{1}, \bar{\beta}_{2}}$.

Thus, the bank state $\tau_{\bar{\beta}_{1}, \bar{\beta}_{2}}$ allows us to obtain the following interconversion relation between the three resources,

$$
\bar{\beta}_{1} \Delta W_{A}+\bar{\beta}_{2} \Delta W_{B}=\Delta W_{S},
$$

while the state of the bank only changes by an infinitesimal amount in terms of $E_{\tau_{\bar{\beta}_{1}, \bar{\beta}_{2}}}$.

\subsection{Local control theory under energetic restrictions}

We now introduce a multi-resource theory describing local control under energetic restrictions. Specifically, we consider the situation in which a quantum system is divided into two well-defined partitions $A$ and $B$, and we can only act on the individual partitions with non-entangling operations, which furthermore need to not increase the energy of the overall system. This kind of simultaneous restrictions on locality and thermodynamics has also been considered in other previous works, see for example Refs. [71-75]. The multi-resource theory is obtained by considering two single-resource theories, the one of entanglement and the one of energy. While this is a well-defined multi-resource theory, it is not straightforward to prove that it is also a reversible theory. Therefore, to provide a first law in this setting, we have to restrict the state-space to a subset of all bipartite density operators.

\subsubsection{Set-up}

Let us consider a bipartite system, whose partitions are labelled as $A$ and $B$, with a non-local Hamiltonian $H_{A B}$ (that is, the two partitions interact with each other, and the ground state of the system is an entangled state). The set of allowed operations of this multi-resource theory is obtained from the intersection of the allowed operations of the following single-resource theories,

- The resource theory of energy. The allowed operations are all the average-energy-non-increasing maps, defined in Sec. 3.4. When the Hamiltonian has non-degenerate ground state $|\mathrm{g}\rangle$, the fixed state of the maps is $\mathcal{F}_{H}=|\mathrm{g}\rangle\langle\mathrm{g}|$. The monotone of this resource theory is $M_{H}(\rho)=\operatorname{Tr}[H \rho]-E_{\mathrm{g}}$, where $E_{\mathrm{g}}$ is the eigenvalue associated with the ground state $|\mathrm{g}\rangle$.

- The resource theory of entanglement. The allowed operations are the asymptotically nonentangling maps [31]. These maps are relevant to us for two reasons. Firstly, all our results hold in the asymptotic limit, and therefore it is reasonable to consider the set of maps which do not create entanglement in this limit. Secondly, this is the only set of operations which provides a reversible theory for entanglement. The monotone is $E_{\mathcal{F}_{\text {sep }}}(\cdot)$, where $\mathcal{F}_{\text {sep }}$ is the set of separable states, invariant under the class of operations.

While the current multi-resource theory is well-defined and meaningful, it is not straightforward to prove whether it is reversible in the sense given in Def. 1. Furthermore, it is known that the relative entropy 
of entanglement, $E_{\mathcal{F}_{\text {sep }}}$, is not additive (or even extensive) for all bipartite density operator. Therefore, if we want to study interconversion of resources in this setting, we need to consider a subset of the state-space (as well as of the invariant set $\mathcal{F}_{\text {sep }}$ ).

In the following we will focus on the simplest example of a multi-resource theory of this kind. The bipartite system is composed by two qubits, so that its Hilbert space is $\mathcal{H}_{A B}=\mathbb{C}^{2} \otimes \mathbb{C}^{2}$. The Hamiltonian of the system is

$$
H_{A B}=E_{0}\left|\Psi_{\text {singlet }}\right\rangle\left\langle\Psi_{\text {singlet }}\right|+E_{1} \Pi_{\text {triplet }},
$$

where $E_{0}<E_{1}$, the ground state is the singlet state,

$$
\left|\Psi_{\text {singlet }}\right\rangle=\frac{1}{\sqrt{2}}(|01\rangle-|10\rangle)
$$

and $\Pi_{\text {triplet }}=\sum_{i=1}^{3}\left|\Psi_{\text {triplet }}^{(i)}\right\rangle\left\langle\Psi_{\text {triplet }}^{(i)}\right|$ is the projector on the triplet subspace, where

$$
\begin{aligned}
& \left|\Psi_{\text {triplet }}^{(1)}\right\rangle=\frac{1}{\sqrt{2}}(|01\rangle+|10\rangle), \\
& \left|\Psi_{\text {triplet }}^{(2)}\right\rangle=\frac{1}{\sqrt{2}}(|00\rangle-|11\rangle), \\
& \left|\Psi_{\text {triplet }}^{(3)}\right\rangle=\frac{1}{\sqrt{2}}(|00\rangle+|11\rangle) .
\end{aligned}
$$

In order to get a reversible multi-resource theory, and therefore to be able to define the interconversion relations, we consider a restricted state-space, given by the following subset of bipartite density operators,

$$
\left.\mathcal{S}_{1}=\left\{\rho \in \mathcal{S}\left(\mathcal{H}_{A B}\right)\left|\rho=\mathrm{p}_{0}\right| \Psi_{\text {singlet }}\right\rangle\left\langle\Psi_{\text {singlet }}\left|+\sum_{i=1}^{3} \mathrm{p}_{i}\right| \Psi_{\text {triplet }}^{(i)}\right\rangle\left\langle\Psi_{\text {triplet }}^{(i)}\right|, \text { with } \mathrm{p}_{0} \geq \frac{1}{2}\right\} .
$$

There are two additional reasons why we are interested in this set of states. First of all, because the relative entropy of entanglement $E_{\mathcal{F}_{\text {sep }}}$ has an analytical expression for states which are diagonal in the Bell basis [76-78] (that here coincides with the energy eigenbasis). Secondly, because it is easy to show, see Eq. (34), that $\mathcal{S}_{1}$ contains the bank states of the theory, that are the interesting ones when it comes to study interconversion. Finally, it is worth noting that the state-space $\mathcal{S}_{1}$ contains all the Gibbs states of the non-local Hamiltonian $H_{A B}$ with positive temperatures. Within this restricted state-space we find the following subset of separable states,

$$
\mathcal{F}_{\text {css }}=\left\{\rho=\frac{1}{2}\left|\Psi_{\text {singlet }}\right\rangle\left\langle\Psi_{\text {singlet }}\left|+\sum_{i=1}^{3} \mathrm{p}_{i}\right| \Psi_{\text {triplet }}^{(i)}\right\rangle\left\langle\Psi_{\text {triplet }}^{(i)}\right|\right\} .
$$

It is worth noticing that the above subset $\mathcal{F}_{\text {css }}$ contains all the closest-separable states to the entangled states in our restricted state-space $\mathcal{S}_{1}$ (see Ref. [78]). As a result, for any state $\rho \in \mathcal{S}_{1}$ we have that

$$
E_{\mathcal{F}_{\text {sep }}}(\rho)=E_{\mathcal{F}_{\text {css }}}(\rho)=1-\mathrm{h}\left(\left\langle\Psi_{\text {singlet }}|\rho| \Psi_{\text {singlet }}\right\rangle\right),
$$

where $\mathrm{h}(\cdot)$ is the binary entropy function. Since our focus is restricted to the sole states in the subset $\mathcal{S}_{1}$, we will now re-define ${ }^{7}$ the set of allowed operations of the multi-resource theory as those energynon-increasing maps which only preserve the subset of separable states $\mathcal{F}_{\text {css }}=\mathcal{F}_{\text {sep }} \cap \mathcal{S}_{1}$. We can define this class of operation as

$\mathcal{C}_{\text {multi }}=\left\{\mathcal{E}: \mathcal{S}\left(\mathcal{H}_{A B}\right) \rightarrow \mathcal{S}\left(\mathcal{H}_{A B}\right) \mid \mathcal{E}\left(\mathcal{F}_{\text {css }}\right) \subseteq \mathcal{F}_{\text {css }}\right.$ and $\left.\operatorname{Tr}\left[\mathcal{E}(\rho) H_{A B}\right] \leq \operatorname{Tr}\left[\rho H_{A B}\right] \forall \rho \in \mathcal{S}\left(\mathcal{H}_{A B}\right)\right\}$

where each $\mathcal{E} \in \mathcal{C}_{\text {multi }}$ is a completely positive and trace preserving map.

\footnotetext{
${ }^{7}$ The modified set of allowed operations makes it easier for us to find a protocol for inter-converting resources. However, we do not exclude the possibility of being able to perform interconversion with the original set of allowed operations, that preserve all separable states. However, finding this protocol might be non-trivial, and could be material of future work.
} 

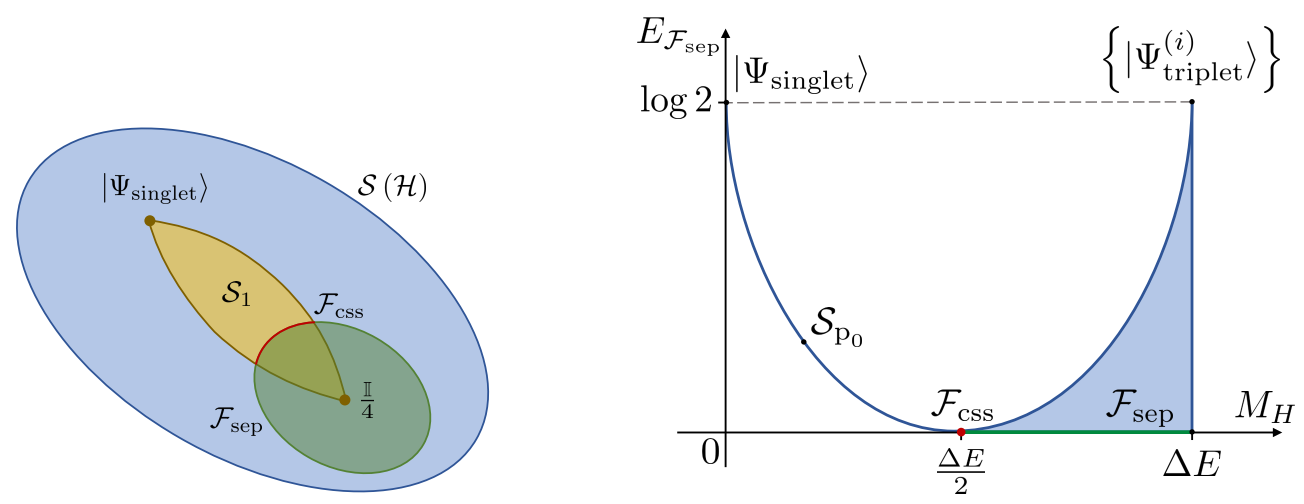

Figure 9: The state-space of the multi-resource theory of local control under energy restrictions. We consider a bipartite system composed by two qubits, with a non-local Hamiltonian given in Eq. (56). Left. The state-space is represented by the blue region $\mathcal{S}(\mathcal{H})$, while the green region is the set of all separable states $\mathcal{F}_{\text {sep }}$, and the red set on its boundary is a subset of separable states $\mathcal{F}_{\text {css }}$, defined in Eq. (62). The yellow region contains every state diagonal in the energy eigenbasis (BellâĂŹs basis). In our simplified example, we restrict the state-space to a portion of the yellow region, that is, to a subset of diagonal states. Specifically, we consider the set $\mathcal{S}_{1}$, containing any states between the singlet and the separable subset $\mathcal{F}_{\text {css. }}$. Right. The diagram represents the set of states diagonal in the energy eigenbasis (blue region). On the left part of the diagram we find the curve associated with the set $\mathcal{S}_{1}$, whose extreme points are the singlet and the invariant set $\mathcal{F}_{\text {css. }}$ On the right hand side, we have all those diagonal states with $\mathrm{p}_{0}<\frac{1}{2}$. The green line is the set of all separable states $\mathcal{F}_{\text {sep. It }}$ is easy to see that, although the set of diagonal states in the Bell's basis is convex, its representation in the diagram has not to be convex, see comments after Lem. 19, in the appendix.

The two batteries we use in the theory store, respectively, energy and entanglement. One can imagine different kinds of energy batteries. For example, we could have that only Alice (or Bob) has access to the battery, which would imply that only one of them can change the energy of the non-local system. However, we prefer to consider a symmetric situation in which both Alice and Bob can interact with the battery. Moreover, we chose the battery to be non-local, so that they are effectively using the same battery, and not two local batteries. Thus, the battery $B_{W}$ is composed by $m$ copies of a two-qubit system with the same Hamiltonian of the main system, that is,

$$
H_{W}=E_{0}\left|\Psi_{\text {singlet }}\right\rangle\left\langle\Psi_{\text {singlet }}\right|+E_{1} \Pi_{\text {triplet }} .
$$

The state of the battery is

$$
\omega_{W}(k)=\left|\Psi_{\text {singlet }}\right\rangle\left\langle\left.\Psi_{\text {singlet }}\right|^{\otimes k} \otimes \mid \Psi_{\text {triplet }}^{(1)}\right\rangle\left\langle\left.\Psi_{\text {triplet }}^{(1)}\right|^{\otimes m-k},\right.
$$

where the excited state $\left|\Psi_{\text {triplet }}^{(1)}\right\rangle$ could be replaced by any other triplet state. Notice that, in order to store/provide energy, we have to change the number of triplet and singlet states contained in the battery, and this can be done locally by both Alice and Bob. Moreover, even if we are changing the energy of the battery, we are not modifying its entanglement, in accord with property M1.

The second battery $B_{E}$ is composed by $\ell$ copies of a two-qubit system with trivial Hamiltonian $H_{E} \propto \mathbb{I}$ (so as to be able to exchange entanglement while preserving the energy of the battery). We choose the state of the battery to be

$$
\omega_{E}(h)=\left|\Psi_{\text {singlet }}\right\rangle\left\langle\left.\Psi_{\text {singlet }}\right|^{\otimes h} \otimes \sigma_{\mathrm{mm}}^{\otimes \ell-h},\right.
$$

where the state $\sigma_{\mathrm{mm}} \in \mathcal{F}_{\mathrm{css}}$, and we take it to be the maximally-mixed state on the subspace spanned by $\left|\Psi_{\text {singlet }}\right\rangle$ and $\left|\Psi_{\text {triplet }}^{(1)}\right\rangle$, that is

$$
\sigma_{\mathrm{mm}}=\frac{1}{2}\left|\Psi_{\text {singlet }}\right\rangle\left\langle\Psi_{\text {singlet }}\left|+\frac{1}{2}\right| \Psi_{\text {triplet }}^{(1)}\right\rangle\left\langle\Psi_{\text {triplet }}^{(1)}\right| .
$$

The change in entanglement is measured by the change in the number of singlet states $h$. 


\subsubsection{Reversibility and the interconversion relation}

In order for the present multi-resource theory to admit an interconversion relation, we first need to show that the asymptotic equivalence property of Def. 1 is satisfied. Let us consider the subset of states $\mathcal{S}_{\mathrm{p}_{0}} \subset \mathcal{S}_{1}$, where $\mathrm{p}_{0}>\frac{1}{2}$, defined as

$$
\mathcal{S}_{\mathrm{p}_{0}}=\left\{\rho \in \mathcal{S}_{1} \mid\left\langle\Psi_{\text {singlet }}|\rho| \Psi_{\text {singlet }}\right\rangle=\mathrm{p}_{0}\right\} .
$$

It is easy to show that all the states in this subset have the same value of the energy and entanglement monotones, which we label $\bar{M}_{H}$ and $\bar{E}_{\mathcal{F}_{\text {css }}}$ respectively. Furthermore, for any two states in this set, we can find an allowed operation in $\mathcal{C}_{\text {multi }}$, see Eq. (64), which maps one into the other. Indeed, consider an ancillary qutrit system described by the state $\eta=\sum_{i=1}^{3} \mathrm{q}_{i}\left|\theta_{i}\right\rangle\left\langle\theta_{i}\right|$, and the global unitary operation $U$ acting on main system and ancilla. The unitary operation maps $\left|\Psi_{\text {triplet }}^{(i)}\right\rangle\left|\theta_{j}\right\rangle$ into $\left|\Psi_{\text {triplet }}^{(j)}\right\rangle\left|\theta_{i}\right\rangle$, for $i, j \in\{1,2,3\}$, and acts trivially on the remaining basis states. Then, the operation $\mathcal{E}_{\eta}(\cdot)=$ $\operatorname{Tr}_{A}\left[U\left(\cdot \otimes \eta_{A}\right) U^{\dagger}\right] \in \mathcal{C}_{\text {multi }}$ maps any state $\rho \in \mathcal{S}_{\mathrm{p}_{0}}$ into the state

$$
\mathcal{E}_{\eta}(\rho)=\mathrm{p}_{0}\left|\Psi_{\text {singlet }}\right\rangle\left\langle\Psi_{\text {singlet }}\left|+\left(1-\mathrm{p}_{0}\right) \sum_{i=1}^{3} \mathrm{q}_{i}\right| \Psi_{\text {triplet }}^{(i)}\right\rangle\left\langle\Psi_{\text {triplet }}^{(i)}\right|,
$$

where the probability distribution $\left\{\mathrm{q}_{i}\right\}_{i=1}^{3}$ is defined by $\eta$. By choosing different ancillary states $\eta$, we can reach different states in $\mathcal{S}_{\mathrm{p}_{0}}$, proving in this way that the resource theory satisfies asymptotic equivalence ${ }^{8}$.

We can now consider the interconversion of energy and entanglement. Together with the two batteries $B_{W}$ and $B_{E}$, one for energy and the other for entropy, we need to use a bank system. One can show that, when diagonal states in the energy eigenbasis are considered, bank states belongs to the set $\mathcal{S}_{1}$ introduced in the previous section. Thus, we describe the bank system using $n \gg 1$ copies of a state $\rho_{\text {in }} \in \mathcal{S}_{\mathrm{p}_{0}}$, where $\mathrm{p}_{0}>\frac{1}{2}$ (the actual form of the state is not relevant, since we can use the allowed operation $\mathcal{E}_{\eta}$ to freely select any state in this set). In order to obtain an interconversion relation, we need to find an allowed operation in $\mathcal{C}_{\text {multi }}$, acting on the global state of bank and batteries, which modifies the state of the batteries (by exchanging resources) while leaving the state of the bank almost unchanged with respect to the relative entropy distance from $\mathcal{S}_{\mathrm{p}_{0}}$.

In appendix $\mathrm{C}$ we provide a protocol which performs the following resource interconversion using an allowed operation $\mathcal{C}_{\text {multi }}$,

$$
\rho_{\mathrm{in}}^{\otimes n} \otimes \omega_{W}(k) \otimes \omega_{E}(h) \stackrel{\text { asympt }}{\longleftrightarrow} \rho_{\mathrm{fin}}^{\otimes n} \otimes \omega_{W}\left(k^{\prime}\right) \otimes \omega_{E}\left(h^{\prime}\right) .
$$

In the above transformation, the initial state of the bank $\rho_{\text {in }}$ is mapped into a state $\rho_{\text {fin }} \in \mathcal{S}_{\mathrm{p}_{0}^{\prime}}$, where $\mathrm{p}_{0}^{\prime}=\mathrm{p}_{0}+O\left(n^{-1}\right)$. The energy battery $B_{W}$ is mapped from the initial state $\omega_{W}(k)$, containing $k$ copies of the ground state of $H_{A B}$, into the final state $\omega_{W}\left(k^{\prime}\right)$ with $k^{\prime}=k+\Delta k$ copies of this ground state, where $\Delta k>0$ is arbitrary big. Likewise, the entanglement battery $B_{E}$ changes from the initial state $\omega_{E}(h)$, containing $h$ singlets, to the final state $\omega_{E}\left(h^{\prime}\right)$ containing $h^{\prime}=h-\log \frac{\mathrm{p}_{0}}{1-\mathrm{p}_{0}} \Delta k$ singlets. From the above transformation one is able to derive an interconversion relation between energy and entanglement,

$$
\Delta W_{W}=-\frac{\Delta E}{\log \frac{\mathrm{p}_{0}}{1-\mathrm{p}_{0}}} \Delta W_{E},
$$

where $\Delta W_{W}=M_{H}\left(\omega_{W}\left(k^{\prime}\right)\right)-M_{H}\left(\omega_{W}(k)\right)$ is the amount of energy exchanged, $\Delta W_{E}=E_{\mathcal{F}_{\text {css }}}\left(\omega_{E}\left(h^{\prime}\right)\right)-$ $E_{\mathcal{F}_{\text {css }}}\left(\omega_{E}(h)\right)$ is the amount of entanglement exchanged, and $\Delta E=E_{1}-E_{0}$ is the energy gap of the Hamiltonian $H_{A B}$. Additionally, we find that the change in monotone $E_{\mathcal{S}_{\mathrm{p}_{0}}}$ between the initial and final global state of the bank is negligible (for $n \rightarrow \infty)$, in accord with property $\mathrm{X} 1$.

\section{Conclusions}

From multiple constraints to a resource theory. With the present work we set the mathematical ground for the development of resource theories with multiple resources able to describe new physical

\footnotetext{
${ }^{8}$ The operation $\mathcal{E}_{\eta}(\cdot)$ we introduce is allowed since we restricted the invariant set $\mathcal{F}_{\text {sep }}$ to $\mathcal{F}_{\text {css }}$. Indeed, the above map would not leave invariant the set of separable states $\mathcal{F}_{\text {sep }}$.
} 
scenarios. Our construction of multi-resource theories is based on the definition of their class of allowed operations. First, we pinpoint the resources that compose the theory, and we introduce the corresponding single-resource theories. Then, we define the set of allowed operations for the multiresource theory as the one composed by the maps in the intersection of the different classes of allowed operations of each single-resource theory, Eq. (11). This construction leaves the theory with multiple invariant sets, some of which are the sets of free states of the relevant single-resource theories. It is worth remarking again that, in multi-constraint theories, there is a difference between the set of free states and the invariant sets (in contrast with the case of single-resource theories), and a multi-resource theory can have multiple invariant sets and no free states, Fig. 2.

Reversibility. Together with the introduction of a general framework for multi-resource theories, we have studied the properties of these reversible theories. In particular, to analyse reversibility when multiple resources are present, we have first introduced the asymptotic equivalence property, see Def. 1. This property implies that a unique monotone can be used to quantify each resource. Furthermore, in the case of single-resource theories, it coincides with the usual notion of reversible rates of conversion. We know of multi-resource theories that satisfy this property, see the two examples provided in Sec. 5. However, it would be interesting to study which of the other, already existing, multi-resource theories satisfy the property of Def. 1 . Ultimately, one would hope to find some general condition according to which a multi-resource theory is reversible, similarly to what has been found in Ref. [33].

The role of batteries. A crucial feature of our framework is the presence of batteries, used to store and quantify the resources exchanged during a state transformation over the main system. While batteries can be defined for single-resource theories as well, they do not seem to play the same fundamental role in that case, since one can quantify the amount of resource contained in a system using the conversion rate, see Def. 14 in appendix A. However, the conversion rate is linked to a change in the number of copies, for example $\rho^{\otimes n} \rightarrow \sigma^{\otimes k}$, where it is implicitly assumed that the remaining $|n-k|$ copies of the system are in a free state. Since the framework allows us to model theories with no free states, we cannot change the number of systems with the allowed operations, and therefore we need to use batteries to quantify the amount of resources. We have seen in this paper what are the main properties for these batteries, primarily property $M 1$, which requires each battery to store one and only one of the resource. It would be interesting to study these systems more carefully, possibly linking them to the kind of batteries used for fluctuation theorems [79-82], which are described by states in a big superposition, so as to always remain uncorrelated from the main system during a state transformation [70, 83]. A different line of research in this direction could involve the study of correlated and entangled batteries, already explored in the setting of the single-resource theory of thermodynamics [71, 84].

Interconversion and further examples. We have studied the interconversion of resources and we have introduced a first law for multi-resource theories, Eq. (45), valid when the theories are reversible and the invariant sets are disjoint. We have provided two examples of theories with a first law, one related to thermodynamics, and the other concerning a theory of local control under energy restriction. In this latter example, we have studied an extremely simplified case, due to the fact that reversibility has not been proved in general for this theory. Due to the high importance of both non-locality and thermodynamics in the field of quantum technology and many-body physics, we believe that a complete analysis of this multi-resource theory would be useful. Furthermore, it would be interesting to know which other multi-resource theories allow for an interconversion relation, and whether it is possible to define interconversion for theories with a different structure of invariant sets, by for instance relaxing the assumptions made on the bank. For example, one could consider bank states from which both resources could in principle be extracted, and forbid such extraction by further constraining the class of allowed operations.

Multiple ways to build a multiple-resource theory. In general, there could be different ways to intersect constraints in order to obtain the same final resource theory, and some of these constructions are a better fit for the analysis presented here than others. For example, the resource theory of thermodynamics equipped with Thermal Operations can be built as the intersection of either (1) the resource theories of information and energy, as we have done in Sec. 4.2, or (2) the resource theories of athermality and coherence [85-87]. However, the most convenient setting for the study of this latter construction is the single-copy regime, since in the many-copy scenario coherence is lost, as this quantity scales sub-linearly in the number of copies of the system considered.

Beyond the asymptotic limit. The concrete results presented here for reversibility and inter- 
conversion of resources are only valid in the asymptotic limit where many independent and identically distributed copies of a system are considered. However, the general framework we introduced to describe resource theories with multiple resources can also be applied to scenarios with a single system. Understanding how resources can be exchanged in the single-copy regime, and studying the corrections to the first law in such a regime are worthwhile questions to pursue. We believe that extending the notion of batteries to the single-shot regime should be the first step toward the definition of a complete framework for multi-resource theories. However, we anticipate that this will be a highly non-trivial task, since in the single-copy case a resource is not generally quantified by a single measure, which complicates the definition of batteries, currently given through property M1. These difficulties are exemplified by the single-shot version of the resource theory of thermodynamics, where the $\alpha$-Rényi divergences from the thermal state are all valid resource measures. A possible way forward in this setting might be the definition of an arbitrary notion of resource, for instance in terms of the number of resourceful states contained in the battery. Otherwise, the fluctuation relations for arbitrary resources [79] and their connection to majorization could be useful conecpts for quantifying resources in the single-shot regime.

\section{Acknowledgement}

We thank the anonymous TQC referees for feedbacks, and Tobias Fritz for detailed comments on a previous version of this manuscript, CS is supported by the EPSRC (grant number EP/L015242/1). LdR acknowledges support from the Swiss National Science Foundation through SNSF project No. 200020_165843 and through the National Centre of Competence in Research Quantum Science and Technology (QSIT), and from the FQXi grant Physics of the observer. CMS is supported by the Engineering and Physical Sciences Research Council (EPSRC) through the doctoral training grant 1652538, and by Oxford-Google DeepMind graduate scholarship. CMS would like to thank the Department of Physics and Astronomy at UCL for their hospitality. PhF acknowledges support from the Swiss National Science Foundation (SNSF) through the Early PostDoc.Mobility Fellowship No. P2EZP2_165239 hosted by the Institute for Quantum Information and Matter (IQIM) at Caltech, from the IQIM which is a National Science Foundation (NSF) Physics Frontiers Center (NSF Grant PHY-1733907), from the Department of Energy Award DE-SC0018407, as well as from the Deutsche Forschungsgemeinschaft (DFG) Research Unit FOR 2724. JO is supported by the Royal Society, and by an EPSRC Established Career Fellowship. We thank the COST Network MP1209 in Quantum Thermodynamics.

\section{Author contributions}

All authors contributed significantly to the ideas behind this work and to the development of the general framework (Sec. 2). CS, LdR and JO developed the results on batteries, bank states and the first law (Secs. 3, 4, 5). CS wrote the proofs and initial draft. 


\section{APPENDIX}

\section{A Reversibility and asymptotic equivalence for single-resource theories}

In this section we show that, for a single-resource theory, the asymptotic equivalence property of Def. 1 is equivalent to the notion of reversibility given in terms of rates of conversion. Let us first introduce the concept of rate of conversion for a single-resource theory, see Ref. [32]. The definition of rate we use coincides with the one used in the literature, with the difference that we are making explicit use of the partial trace and of the addition of free states. In fact, we prefer not to include these operations within the set $\mathcal{C}$, as we want the allowed operations to preserve the number of copies of the system they act over (with the exception of sub-linear ancillae).

Definition 14. Consider a single-resource theory with allowed operations $\mathcal{C}$ and free states $\mathcal{F}$, and two states $\rho, \sigma \in \mathcal{S}(\mathcal{H})$. We define the rate of conversion from $\rho$ to $\sigma$ as

$$
\begin{gathered}
R(\rho \rightarrow \sigma)=\sup \left\{\frac{k_{n}}{n} \mid \text { either } \lim _{n \rightarrow \infty}\left(\min _{\tilde{\mathcal{E}}_{n}}\left\|\operatorname{Tr}_{n-k_{n}}\left[\tilde{\mathcal{E}}_{n}\left(\rho^{\otimes n}\right)\right]-\sigma^{\otimes k_{n}}\right\|_{1}\right)=0\right. \\
\text { or } \lim _{n \rightarrow \infty}\left(\min _{\tilde{\mathcal{E}}_{k_{n}}}\left\|\tilde{\mathcal{E}}_{k_{n}}\left(\rho^{\otimes n} \otimes \gamma_{k_{n}-n}\right)-\sigma^{\otimes k_{n}}\right\|_{1}\right)=0, \\
\text { where } \left.\gamma_{k_{n}-n} \in \mathcal{F}^{\left(k_{n}-n\right)}\right\} .
\end{gathered}
$$

where the maps $\tilde{\mathcal{E}}_{n}$ have been defined in Eq. (13), and they are of the form $\tilde{\mathcal{E}}_{n}(\cdot)=\operatorname{Tr}_{A}\left[\mathcal{E}_{n}\left(\cdot \otimes \eta_{n}^{(A)}\right)\right]$, with $\mathcal{E}_{n} \in \mathcal{C}^{(n+o(n))}$ and $\eta_{n}^{(A)} \in \mathcal{S}\left(\mathcal{H}^{\otimes o(n)}\right)$.

Now that the notion of rate is defined, we introduce the concept of reversible single-resource theory,

Definition 15. A single-resource theory with allowed operations $\mathcal{C}$ and free states $\mathcal{F}$ is reversible if, given any non-free states $\rho, \sigma \in \mathcal{S}(\mathcal{H})$, the rate of conversion from $\rho$ to $\sigma$ is such that $R(\rho \rightarrow \sigma) \in(0, \infty)$, and $R(\rho \rightarrow \sigma) R(\sigma \rightarrow \rho)=1$.

The above notion of reversibility is based on the rates of conversion between two resourceful states. However, it is not clear how to extend Def. 14 to the case of multiple resources, since the set of free states might be empty for multi-resource theories. For this reason, we have introduced the property of asymptotic equivalence in Sec. 3.1. This property also apply to the single-resource theory case, when $m=1$.

Now we want to show that Defs. 1 and 15, for a single-resource theory, coincide. First, let us introduce a function $f: \mathcal{S}\left(\mathcal{H}^{\otimes n}\right) \rightarrow \mathbb{R}$ (more formally, a family of functions) with the following properties,

SM1 For each $n \in \mathbb{N}$, the function $f$ is monotonic under the set of allowed operations $\mathcal{C}^{(n)}$, that is

$$
f\left(\mathcal{E}_{n}\left(\rho_{n}\right)\right) \leq f\left(\rho_{n}\right), \quad \forall \rho_{n} \in \mathcal{S}\left(\mathcal{H}^{\otimes n}\right), \forall \mathcal{E}_{n} \in \mathcal{C}^{(n)}
$$

SM2 For each $n \in \mathbb{N}$, the function $f$ is equal to 0 for all states $\gamma_{n} \in \mathcal{F}^{(n)}$, that is

$$
f\left(\gamma_{n}\right)=0, \quad \forall \gamma_{n} \in \mathcal{S}\left(\mathcal{H}^{\otimes n}\right) .
$$

SM3 The function $f$ is asymptotic continuous.

SM4 The function $f$ is monotonic under partial tracing, that is

$$
f\left(\operatorname{Tr}_{k}\left[\rho_{n}\right]\right) \leq f\left(\rho_{n}\right), \quad \forall n, k \in \mathbb{N}, k<n, \forall \rho_{n} \in \mathcal{S}\left(\mathcal{H}^{\otimes n}\right) .
$$

SM5 For each $n, k \in \mathbb{N}$, the function $f$ is sub-additive, that is

$$
f\left(\rho_{n} \otimes \rho_{k}\right) \leq f\left(\rho_{n}\right)+f\left(\rho_{k}\right), \quad \forall \rho_{n} \in \mathcal{S}\left(\mathcal{H}^{\otimes n}\right), \forall \rho_{k} \in \mathcal{S}\left(\mathcal{H}^{\otimes k}\right) .
$$


SM6 For any given sequence of states $\left\{\rho_{n} \in \mathcal{S}\left(\mathcal{H}^{\otimes n}\right)\right\}$, the function $f$ scales sub-extensively, that is, $f\left(\rho_{n}\right)=O(n)$.

Notice that property SM6 implies that the function $f$ is regularisable. Furthermore, the value of $f$ is preserved if we add free states, that is,

$$
f\left(\rho_{n} \otimes \gamma_{k}\right)=f\left(\rho_{n}\right), \quad \forall \rho_{n} \in \mathcal{S}\left(\mathcal{H}^{\otimes n}\right), \forall \gamma_{k} \in \mathcal{F}^{(k)},
$$

which follows from properties SM2, SM4, and SM5.

The first lemma we introduce show that the rate of conversion of a reversible single-resource theory is linked to the function $f$ satisfying the above properties. Notice that this proof is analogous to the one of Ref. [62], with the difference that we are allowing for the presence of a sub-linear ancilla in the definition of rate, following the notion of "seed regularisation" introduced in Ref. [23, Sec. 9].

Lemma 16. Consider a reversible resource theory with allowed operations $\mathcal{C}$ and free states $\mathcal{F}$, and the function $f$ satisfying SM1 - SM6. Then, for all non-free states $\rho, \sigma \in \mathcal{S}(\mathcal{H})$, we have that

$$
R(\rho \rightarrow \sigma)=\frac{f^{\infty}(\rho)}{f^{\infty}(\sigma)}
$$

Proof. Let consider $\rho$ and $\sigma$ such that $R(\rho \rightarrow \sigma) \leq 1$ (the proof of the other case is equivalent). Then, there exists a sequence of operations $\left\{\tilde{\mathcal{E}}_{n}\right\}$ of the form given in Eq. (13) such that

$$
\lim _{n \rightarrow \infty}\left\|\operatorname{Tr}_{n-k_{n}}\left[\tilde{\mathcal{E}}_{n}\left(\rho^{\otimes n}\right)\right]-\sigma^{\otimes k_{n}}\right\|_{1}=0
$$

where $\lim _{n \rightarrow \infty} \frac{k_{n}}{n}=R(\rho \rightarrow \sigma)$. If we use the asymptotic continuity of the function $f$, property SM3, we obtain

$$
f\left(\operatorname{Tr}_{n-k_{n}}\left[\tilde{\mathcal{E}}_{n}\left(\rho^{\otimes n}\right)\right]\right)=f\left(\sigma^{\otimes k_{n}}\right)+o\left(k_{n}\right) .
$$

Let us now consider the Ihs of the above equation. Using the properties of the monotone $f$, together with the definition of $\tilde{\mathcal{E}}_{n}$ in terms of sub-linear ancillae and allowed operations, we can prove the following chain of inequalities,

$$
\begin{aligned}
f\left(\operatorname{Tr}_{n-k_{n}}\left[\tilde{\mathcal{E}}_{n}\left(\rho^{\otimes n}\right)\right]\right) & \leq f\left(\tilde{\mathcal{E}}_{n}\left(\rho^{\otimes n}\right)\right)=f\left(\operatorname{Tr}_{A}\left[\mathcal{E}_{n}\left(\rho^{\otimes n} \otimes \eta_{n}^{(A)}\right)\right]\right) \leq f\left(\mathcal{E}_{n}\left(\rho^{\otimes n} \otimes \eta_{n}^{(A)}\right)\right) \\
& \leq f\left(\rho^{\otimes n} \otimes \eta_{n}^{(A)}\right) \leq f\left(\rho^{\otimes n}\right)+f\left(\eta_{n}^{(A)}\right) \leq f\left(\rho^{\otimes n}\right)+o(n)
\end{aligned}
$$

where the first and second inequalities follow from property SM4, the equality follows from the definition of $\tilde{\mathcal{E}}_{n}$, see Eq. (13), the third inequality follows from monotonicity under allowed operations, property SM1, the forth inequality from sub-additivity, property SM5, and the last one from the fact that the ancillary system is sub-linear in $n$ together with property SM6. Thus, combining the last two equations, we get

$$
f\left(\rho^{\otimes n}\right) \geq f\left(\sigma^{\otimes k_{n}}\right)+o(n) .
$$

We can now divide the left and right hand side of the above equation by $n$, obtaining

$$
\frac{1}{n} f\left(\rho^{\otimes n}\right) \geq \frac{k_{n}}{n} \frac{1}{k_{n}} f\left(\sigma^{\otimes k_{n}}\right)+o(1) .
$$

By taking the limit of $n \rightarrow \infty$, and using the fact that $f$ is regularisable (which follows from property SM6) together with the definition of rate, we get

$$
f^{\infty}(\rho) \geq R(\rho \rightarrow \sigma) f^{\infty}(\sigma) .
$$

We can also consider the reverse transformation, mapping $n$ copies of the state $\sigma$ into $k_{n}^{\prime}$ copies of $\rho$. Using the same steps used above, together with the fact that the monotone $f$ is equal to zero over free states, property SM2, we can show that

$$
f^{\infty}(\sigma) \geq R(\sigma \rightarrow \rho) f^{\infty}(\rho) .
$$

If we now use the reversibility property, which implies $R(\sigma \rightarrow \rho)=\frac{1}{R(\rho \rightarrow \sigma)}$, we find that

$$
\frac{f^{\infty}(\rho)}{f^{\infty}(\sigma)} \geq R(\rho \rightarrow \sigma) \geq \frac{f^{\infty}(\rho)}{f^{\infty}(\sigma)}
$$

which proves the lemma. 
Furthermore, we introduce a second small lemma, that can be found in Ref. [88, Prop. 13],

Lemma 17. Given a regularisable function $f: \mathcal{S}\left(\mathcal{H}^{\otimes n}\right) \rightarrow \mathbb{R}$, the regularised version is extensive,

$$
f^{\infty}\left(\rho^{\otimes k}\right)=k f^{\infty}(\rho), \forall \rho \in \mathcal{S}(\mathcal{H}), \forall k \in \mathbb{N} .
$$

Proof. Consider a function $h: \mathbb{R} \rightarrow \mathbb{R}$, such that $\lim _{n \rightarrow \infty} h(n)=L<\infty$. This is equivalent to say that

$$
\forall \epsilon>0, \exists c \in \mathbb{R}:|h(n)-L|<\epsilon, \forall n>c .
$$

Let us now consider an invertible function $g: \mathbb{R} \rightarrow \mathbb{R}$, and consider $m \in \mathbb{R}$ such that $n=g(m)$. Then, we can rewrite Eq. (89) as

$$
\forall \epsilon>0, \exists c \in \mathbb{R}:|h(g(m))-L|<\epsilon, \forall g(m)>c,
$$

and by defining $\tilde{c}=g^{-1}(c)$, we get

$$
\forall \epsilon>0, \exists \tilde{c} \in \mathbb{R}:|h(g(m))-L|<\epsilon, \forall m>\tilde{c} .
$$

Therefore, we have $\lim _{m \rightarrow \infty} h(g(m))=L$.

If we choose $h(n)=\frac{1}{n} f\left(\rho^{\otimes n}\right)$, whose limit is $L=f^{\infty}(\rho)$, and we use the reversible function $g(m)=k \cdot m$ where $k \in \mathbb{N}$ is fixed, we get

$$
f^{\infty}(\rho)=\lim _{m \rightarrow \infty} \frac{1}{k \cdot m} f\left(\rho^{\otimes k \cdot m}\right)=\frac{1}{k} \lim _{m \rightarrow \infty} \frac{1}{m} f\left(\left(\rho^{\otimes k}\right)^{\otimes m}\right)=\frac{1}{k} f^{\infty}\left(\rho^{\otimes k}\right),
$$

which proves the lemma.

We can now show that a single-resource theory which is reversible also satisfies the asymptotic equivalence property, and vice versa.

Theorem 18. Consider the resource theory with allowed operations $\mathcal{C}$ and free states $\mathcal{F}$. If the theory is reversible, then it satisfies the asymptotic equivalence property with respect to a function $f$ satisfying the properties SM1 - SM6, and viceversa.

Proof. (a) Let us first assume that the theory is reversible. Then, if we consider two non-free states $\rho, \sigma \in \mathcal{S}(\mathcal{H})$ such that $f^{\infty}(\rho)=f^{\infty}(\sigma)$, and we use Lem. 16, we find that the rate of conversion is $R(\rho \rightarrow \sigma)=1$. Then, there exists a sequence of operations $\left\{\tilde{\mathcal{E}}_{n}\right\}$ that approach this limit in one of two ways. In one case, we have

$$
\left\|\operatorname{Tr}_{n-k_{n}}\left[\tilde{\mathcal{E}}_{n}\left(\rho^{\otimes n}\right)\right]-\sigma^{\otimes k_{n}}\right\|_{1} \rightarrow 0
$$

Notice that, since we have $\frac{k_{n}}{n} \rightarrow 1$, it follows that $n-k_{n}=o(n)$. Then, the above equation coincides with the second part of Def. 1, where we are mapping $\rho^{\otimes k_{n}}$ into $\sigma^{\otimes k_{n}}$, and the sub-linear ancilla is $\eta_{n}^{(A)}=\eta_{n}^{(A)} \otimes \rho^{n-k_{n}}$, where $\eta_{n}^{(A)}$ is completely arbitrary, and come from the definition of $\tilde{\mathcal{E}}_{n}$. Alternatively, we can have that the sequence of maps is such that

$$
\left\|\tilde{\mathcal{E}}_{k_{n}}\left(\rho^{\otimes n} \otimes \gamma_{k_{n}-n}\right)-\sigma^{\otimes k_{n}}\right\|_{1} \rightarrow 0 .
$$

We now use the monotonicity of the trace distance under discarding subsystems to obtain

$$
\left\|\operatorname{Tr}_{k_{n}-n}\left[\tilde{\mathcal{E}}_{k_{n}}\left(\rho^{\otimes n} \otimes \gamma_{k_{n}-n}\right)\right]-\sigma^{\otimes n}\right\|_{1} \rightarrow 0 .
$$

Again, the above equation coincides with the second part of Def. 1, where we are mapping $\rho^{\otimes n}$ into $\sigma^{\otimes n}$, and the sub-linear ancilla is $\eta_{n}^{\prime(A)}=\eta_{n}^{(A)} \otimes \gamma_{k_{n}-n}$. This proves the validity of one direction of the asymptotic equivalence property. To prove the other direction (existence of a sequence of maps implies same value of the monotone on the two states), we can use the fact that, if there exists a sequence of maps $\left\{\tilde{\mathcal{E}}_{n}\right\}$ sending $\rho^{\otimes n}$ into $\sigma^{\otimes n}$, then the rate of conversion is $R(\rho \rightarrow \sigma)=1$. Then, with the help of Lem. 16, which is valid for reversible theories, we obtain that $f^{\infty}(\rho)=f^{\infty}(\sigma)$. This proves the other direction of the asymptotic equivalence property. 
(b) Let now assume that the theory satisfies the asymptotic equivalence property. Consider any two non-free states $\rho, \sigma \in \mathcal{S}(\mathcal{H})$, and suppose that $f^{\infty}(\rho) \leq f^{\infty}(\sigma)$ (in the other case, the proof would follow analogously to the one we are presenting). Take $n, k \in \mathbb{N}$ such that $n f^{\infty}(\rho)=k f^{\infty}(\sigma)$, and let us use the extensivity of $f^{\infty}$, Lem. 17. Then, we have $f^{\infty}\left(\rho^{\otimes n}\right)=f^{\infty}\left(\sigma^{\otimes k}\right)$. Using the property of the function $f$ shown in Eq. (78), we find that

$$
f^{\infty}\left(\rho^{\otimes n}\right)=f^{\infty}\left(\sigma^{\otimes k} \otimes \gamma_{n-k}\right),
$$

where we add the free state $\gamma_{n-k} \in \mathcal{F}^{(n-k)}$ to the right hand side since $n \geq k$. Then, we can use the asymptotic equivalence property, which implies the existence of a sequence of maps $\left\{\tilde{\mathcal{E}}_{m \cdot n}\right\}_{m}$, see Eq. 13, such that

$$
\lim _{m \rightarrow \infty}\left\|\mathcal{E}_{m \cdot n}\left(\rho^{\otimes m \cdot n}\right)-\sigma^{\otimes m \cdot k} \otimes \gamma_{n-k}^{\otimes m}\right\|_{1}=0
$$

If we use the monotonicity of the trace distance under partial tracing, we find that

$$
\lim _{m \rightarrow \infty}\left\|\operatorname{Tr}_{m \cdot(n-k)}\left[\mathcal{E}_{m \cdot n}\left(\rho^{\otimes m \cdot n}\right)\right]-\sigma^{\otimes m \cdot k}\right\|_{1}=0 .
$$

The existence of this sequence of maps implies that the rate of conversion $R(\rho \rightarrow \sigma) \geq \frac{k}{n}$. At the same time, we can use asymptotic equivalence to find a sequence of maps $\left\{\tilde{\mathcal{E}}_{m \cdot n}^{\prime}\right\}_{m}$ performing the reverse process. Using a similar argument to the one presented above, we find that $R(\sigma \rightarrow \rho) \geq \frac{n}{k}$. As a result, we find that the product of the forward and reverse rates of conversion is $R(\rho \rightarrow \sigma) R(\sigma \rightarrow \rho) \geq 1$. However, this product cannot be higher than one, as otherwise we would be able to perform a cyclic transformation turning free states intro resourceful one, which is forbidden under allowed operations, see also Ref. [28]. Therefore, we find that $R(\rho \rightarrow \sigma) R(\sigma \rightarrow \rho)=1$, which closes the proof.

\section{B Convex boundary and bank states}

In the following, we consider the case of a two-resource theory $\mathrm{R}_{\text {multi }}$ defined on the Hilbert space $\mathcal{H}$. The set of allowed operations is $\mathcal{C}_{\text {multi }}=\mathcal{C}_{1} \cap \mathcal{C}_{2}$, where each $\mathcal{C}_{i}$ is a subset of the set of all CPTP maps that leave the set of states $\mathcal{F}_{i}$ invariant, $i=1,2$. We ask the resource theory $\mathrm{R}_{\text {multi }}$ to satisfy the asymptotic equivalence property with respect to the monotones $E_{\mathcal{F}_{1}}$ and $E_{\mathcal{F}_{2}}$. Furthermore, we assume that the two invariant sets satisfy the properties F1-F5. Thus, it follows from Thm. 5 and Prop. 6 that the two monotones $E_{\mathcal{F}_{1}}^{\infty}$ and $E_{\mathcal{F}_{2}}^{\infty}$ uniquely quantify the resources in this theory. As a result, we can represent the state-space of $\mathrm{R}_{\text {multi }}$ in a two-dimensional diagram, as shown in Fig. 3.

We choose the two invariant sets of the theory to be disjoints, i.e., $\mathcal{F}_{1} \cap \mathcal{F}_{2}=\emptyset$, and we focus on the set of bank states $\mathcal{F}_{\text {bank }} \subset \mathcal{S}(\mathcal{H})$. Since in this section we are not making any assumption on the additivity (or extensivity) of the monotones $E_{\mathcal{F}_{i}}$ 's, we have that the set of bank states is here defined as

$$
\begin{aligned}
\mathcal{F}_{\text {bank }}=\{\rho \in \mathcal{S}(\mathcal{H}) \mid \forall \sigma \in \mathcal{S}(\mathcal{H}), & E_{\mathcal{F}_{1}}^{\infty}(\sigma)>E_{\mathcal{F}_{1}}^{\infty}(\rho) \text { or } \\
& E_{\mathcal{F}_{2}}^{\infty}(\sigma)>E_{\mathcal{F}_{2}}^{\infty}(\rho) \text { or } \\
& \left.E_{\mathcal{F}_{1}}^{\infty}(\sigma)=E_{\mathcal{F}_{1}}^{\infty}(\rho) \text { and } E_{\mathcal{F}_{2}}^{\infty}(\sigma)=E_{\mathcal{F}_{2}}^{\infty}(\rho)\right\} .
\end{aligned}
$$

Notice that this set coincides with the one of Eq. (34) when property F5b is satisfied, and therefore the results we obtain in this appendix apply to Sec. 4 as well. It is easy to show that $E_{\mathcal{F}_{2}}^{\infty}(\rho)>E_{\mathcal{F}_{2}}^{\infty}\left(\mathcal{F}_{2}\right)=$ $0 \forall \rho \in \mathcal{F}_{1}$, and similarly $E_{\mathcal{F}_{1}}^{\infty}(\rho)>E_{\mathcal{F}_{1}}^{\infty}\left(\mathcal{F}_{1}\right)=0 \forall \rho \in \mathcal{F}_{2}$. Moreover, inside both invariant sets $\mathcal{F}_{1}$ and $\mathcal{F}_{2}$ we can find a subset of states with minimum value of the monotones $E_{\mathcal{F}_{2}}^{\infty}$ and $E_{\mathcal{F}_{1}}^{\infty}$, respectively. We define these sets as

$$
\begin{aligned}
& \mathcal{F}_{1, \text { min }}=\left\{\sigma \in \mathcal{F}_{1} \mid E_{\mathcal{F}_{2}}^{\infty}(\sigma)=\min _{\rho \in \mathcal{F}_{1}} E_{\mathcal{F}_{2}}^{\infty}(\rho)\right\} \subseteq \mathcal{F}_{1}, \\
& \mathcal{F}_{2, \text { min }}=\left\{\sigma \in \mathcal{F}_{2} \mid E_{\mathcal{F}_{1}}^{\infty}(\sigma)=\min _{\rho \in \mathcal{F}_{2}} E_{\mathcal{F}_{1}}^{\infty}(\rho)\right\} \subseteq \mathcal{F}_{2} .
\end{aligned}
$$

Given these two subsets, we can then define the following real intervals,

$$
\begin{aligned}
& I_{1}=\left[E_{\mathcal{F}_{1}}^{\infty}\left(\mathcal{F}_{1}\right)=0 ; E_{\mathcal{F}_{1}}^{\infty}\left(\mathcal{F}_{2, \text { min }}\right)\right], \\
& I_{2}=\left[E_{\mathcal{F}_{2}}^{\infty}\left(\mathcal{F}_{2}\right)=0 ; E_{\mathcal{F}_{2}}^{\infty}\left(\mathcal{F}_{1, \text { min }}\right)\right] .
\end{aligned}
$$

In what follows, we make use of the following two properties of the monotones $E_{\mathcal{F}_{i}}^{\infty}$ 's, 


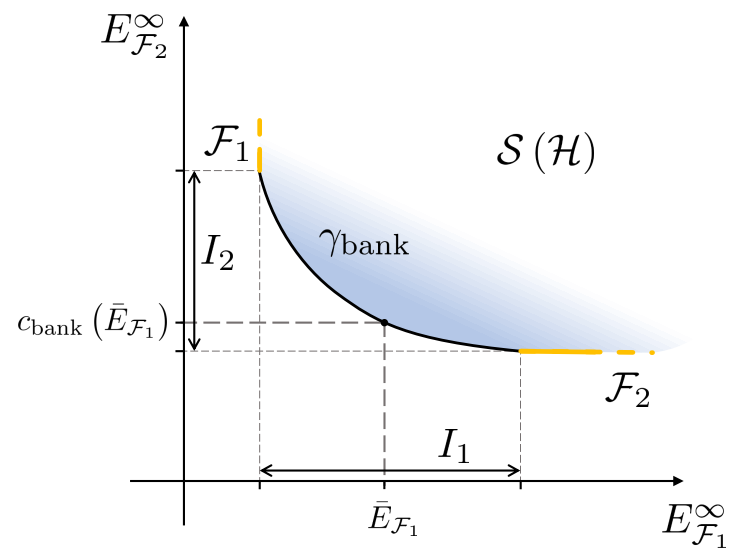

Figure 10: We represent part of the state-space $\mathcal{S}(\mathcal{H})$ in the $E_{\mathcal{F}_{1}}^{\infty}-E_{\mathcal{F}_{2}}^{\infty}$ diagram. In the figure, the green segment is the invariant set $\mathcal{F}_{1}$, the yellow one is $\mathcal{F}_{2}$, and the black curve connecting these two segments is $\gamma_{\text {bank, }}$, the curve of bank states of the theory, see Eq. (104). On the $E_{\mathcal{F}_{1}}^{\infty}$-axis we highlight the interval $I_{1}$ defined in Eq. (102), and similarly for the interval $I_{2}$ on the $E_{\mathcal{F}_{2}}^{\infty}$-axis. Furthermore, the action of the function $c_{\text {bank }}: I_{1} \rightarrow I_{2}$, defined in Eq. (105), is shown for the input value $\bar{E}_{\mathcal{F}_{1}}$.

- Asymptotic continuity, which follows from the assumptions F1-F5 over the sets $\mathcal{F}_{i}$ 's, as shown in Refs. [33, 89].

- Convexity, which follows from the assumptions F2 and F4 over the sets $\mathcal{F}_{i}$ 's, as shown in Ref. [88], Prop. 13.

We can now state the following lemma, concerning the value of the monotones $E_{\mathcal{F}_{i}}^{\infty}$ 's for bank states.

Lemma 19. Consider the multi-resource theory $R_{\text {multi }}$ with allowed operations $\mathcal{C}_{\text {multi, }}$ and invariant sets $\mathcal{F}_{1}$ and $\mathcal{F}_{2}$ which satisfy properties $F 1-F 5$, and $\mathcal{F}_{1} \cap \mathcal{F}_{2}=\emptyset$. If the theory satisfies the asymptotic equivalence property with respect to the monotones $E_{\mathcal{F}_{1}}$ and $E_{\mathcal{F}_{2}}$, then for all bank states $\rho \in \mathcal{F}_{\text {bank }}$ we have that $E_{\mathcal{F}_{1}}^{\infty}(\rho) \in I_{1}$ and $E_{\mathcal{F}_{2}}^{\infty}(\rho) \in I_{2}$.

Proof. Suppose, for example, that there exists a bank state $\rho \in \mathcal{F}_{\text {bank }}$ such that $E_{\mathcal{F}_{1}}^{\infty}(\rho) \notin I_{1}$, that is, $\exists \sigma \in \mathcal{F}_{2, \text { min }}$ such that $E_{\mathcal{F}_{1}}^{\infty}(\sigma)<E_{\mathcal{F}_{1}}^{\infty}(\rho)$. By definition of $\mathcal{F}_{2}$ we also have that $E_{\mathcal{F}_{2}}^{\infty}(\sigma) \leq E_{\mathcal{F}_{2}}^{\infty}(\rho)$. These two inequalities, however, contradict the fact that $\rho$ is a bank state, see Eq. (99), and conclude the proof.

It is easy to show that for all $\bar{E}_{\mathcal{F}_{1}} \in I_{1}$ there exists (at least) one state $\rho \in \mathcal{S}(\mathcal{H})$ such that $E_{\mathcal{F}_{1}}^{\infty}(\rho)=\bar{E}_{\mathcal{F}_{1}}$, and the same holds for $I_{2}$. The proof that $\forall \bar{E}_{\mathcal{F}_{1}} \in I_{1}, \exists \rho \in \mathcal{S}(\mathcal{H}): E_{\mathcal{F}_{1}}^{\infty}(\rho)=\bar{E}_{\mathcal{F}_{1}}$ follows from two facts: $(i) \mathcal{S}(\mathcal{H})$ is a compact and path-connected set, and therefore its image under the (asymptotic) continuous function $E_{\mathcal{F}_{1}}^{\infty}$ is a compact and path-connected set in $\mathbb{R}$, that is, a closed and bounded interval $I_{1, \mathcal{S}(\mathcal{H})}$, and $(i i) I_{1} \subseteq I_{1, \mathcal{S}(\mathcal{H})}$.

Let us now define, in the $E_{\mathcal{F}_{1}}^{\infty}-E_{\mathcal{F}_{2}}^{\infty}$ diagram, the curve of bank states, which lies on part of the boundary of the state-space, as per definition in Eq. (99). The curve is defined as

$$
\gamma_{\text {bank }}=\left\{\left(E_{\mathcal{F}_{1}}^{\infty}(\rho), E_{\mathcal{F}_{2}}^{\infty}(\rho)\right) \mid \rho \in \mathcal{F}_{\text {bank }}\right\},
$$

where $\mathcal{F}_{\text {bank }}$ is the set of bank states of the theory. It is easy to see that this curve is completely contained within the subset of $\mathbb{R}^{2}$ given by $I_{1} \times I_{2}$. Together with this curve, we can introduce the real-valued function $c_{\text {bank }}: I_{1} \rightarrow I_{2}$, defined as

$$
c_{\text {bank }}\left(E_{\mathcal{F}_{1}}\right)=\text { if }\left(\exists P \in \gamma_{\text {bank }} \text { such that } P[0]=E_{\mathcal{F}_{1}}\right) \text { return } P[1] .
$$

Essentially, this function checks the first element of the tuples in $\gamma_{\text {bank, }}$, and returns the second element of the tuple whose first element is equal to $E_{\mathcal{F}_{1}}$. Since $I_{1}$ is a closed interval in $\mathbb{R}$, we have that for all $E_{\mathcal{F}_{1}} \in I_{1}$, the function $c_{\text {bank }}$ is well-defined. See Fig. 10 for the representation of the above curve of bank states in the resource diagram of the theory. 
We will now prove the following two propositions, which assure that the monotone $f_{\text {bank }}^{\bar{E}_{\mathcal{F}_{1}}, \bar{E}_{\mathcal{F}_{2}}}$ of Eq. (37) satisfies the property B2. This first proposition essentially tells us that the function $c_{\text {bank }}$ is monotonic decreasing.

Proposition 20. For all $P_{A}, P_{B} \in \gamma_{\text {bank, }}$, where $P_{A}=\left(E_{\mathcal{F}_{1}}^{(A)}, E_{\mathcal{F}_{2}}^{(A)}\right)$ and $P_{B}=\left(E_{\mathcal{F}_{1}}^{(B)}, E_{\mathcal{F}_{2}}^{(B)}\right)$, we have that

$$
E_{\mathcal{F}_{1}}^{(A)}<E_{\mathcal{F}_{1}}^{(B)} \Leftrightarrow E_{\mathcal{F}_{2}}^{(A)}>E_{\mathcal{F}_{2}}^{(B)}
$$

Proof. We prove the propositions in a single direction, as the other follows in analogue manner. Suppose that $E_{\mathcal{F}_{1}}^{(A)}<E_{\mathcal{F}_{1}}^{(B)}$, and consider the states $\rho_{A}, \rho_{B} \in \mathcal{F}_{\text {bank }}$ such that $E_{\mathcal{F}_{1}}^{\infty}\left(\rho_{A}\right)=E_{\mathcal{F}_{1}}^{(A)}$, and $E_{\mathcal{F}_{1}}^{\infty}\left(\rho_{B}\right)=$ $E_{\mathcal{F}_{1}}^{(B)}$. Since $\rho_{B}$ belongs to the set of bank states, we have that one of the following conditions, see Eq. (34), has to be satisfied for all states $\sigma \in \mathcal{S}(\mathcal{H})$,

1. $E_{\mathcal{F}_{1}}^{\infty}(\sigma)>E_{\mathcal{F}_{1}}^{\infty}\left(\rho_{B}\right)$.

2. $E_{\mathcal{F}_{2}}^{\infty}(\sigma)>E_{\mathcal{F}_{2}}^{\infty}\left(\rho_{B}\right)$.

3. $E_{\mathcal{F}_{1}}^{\infty}(\sigma)=E_{\mathcal{F}_{1}}^{\infty}\left(\rho_{B}\right)$ and $E_{\mathcal{F}_{2}}^{\infty}(\sigma)=E_{\mathcal{F}_{2}}^{\infty}\left(\rho_{B}\right)$.

Let us then take $\sigma=\rho_{A}$. In this case, options 1 and 3 are not possible, since they contradict the hypothesis. Therefore, option 2 has to be valid, which implies that $E_{\mathcal{F}_{2}}^{\infty}\left(\rho_{A}\right)>E_{\mathcal{F}_{2}}^{\infty}\left(\rho_{B}\right)$. In a similar manner, if $E_{\mathcal{F}_{1}}^{(A)}=E_{\mathcal{F}_{1}}^{(B)}$, the only possible option for $\rho_{B}$ would have been $E_{\mathcal{F}_{2}}^{\infty}\left(\rho_{A}\right)=E_{\mathcal{F}_{2}}^{\infty}\left(\rho_{B}\right)$, which concludes the proof.

The second propositions tells us, instead, that the function $c_{\text {bank }}$ is convex.

Proposition 21. For all $P_{A}, P_{B} \in \gamma_{\text {bank }}$, where $P_{A}=\left(E_{\mathcal{F}_{1}}^{(A)}, E_{\mathcal{F}_{2}}^{(A)}\right)$ and $P_{B}=\left(E_{\mathcal{F}_{1}}^{(B)}, E_{\mathcal{F}_{2}}^{(B)}\right)$, and for all $\lambda \in[0,1]$, there exists a $P_{C} \in \gamma_{\text {bank }}$, where $P_{C}=\left(E_{\mathcal{F}_{1}}^{(C)}, E_{\mathcal{F}_{2}}^{(C)}\right)$, such that

$$
\begin{aligned}
& E_{\mathcal{F}_{1}}^{(C)}=\lambda E_{\mathcal{F}_{1}}^{(A)}+(1-\lambda) E_{\mathcal{F}_{1}}^{(B)}, \\
& E_{\mathcal{F}_{2}}^{(C)} \leq \lambda E_{\mathcal{F}_{2}}^{(A)}+(1-\lambda) E_{\mathcal{F}_{2}}^{(B)}
\end{aligned}
$$

Proof. Let us consider, without losing in generality, that $E_{\mathcal{F}_{1}}^{(A)}<E_{\mathcal{F}_{1}}^{(B)}$, and take $\rho_{C} \in \mathcal{F}_{\text {bank }}$ such that $E_{\mathcal{F}_{1}}^{\infty}\left(\rho_{C}\right)=\lambda E_{\mathcal{F}_{1}}^{(A)}+(1-\lambda) E_{\mathcal{F}_{1}}^{(B)}$. This state always exists since $I_{1}$ is a closed interval (and therefore is path-connected). Let us now define $\rho_{A}, \rho_{B} \in \mathcal{F}_{\text {bank }}$ such that $E_{\mathcal{F}_{1}}^{\infty}\left(\rho_{A}\right)=E_{\mathcal{F}_{1}}^{(A)}$, and $E_{\mathcal{F}_{1}}^{\infty}\left(\rho_{B}\right)=E_{\mathcal{F}_{1}}^{(B)}$. By convexity of the regularised relative entropy distance $E_{\mathcal{F}_{1}}^{\infty}$, it follows that

$$
E_{\mathcal{F}_{1}}^{\infty}\left(\rho_{C}\right)=\lambda E_{\mathcal{F}_{1}}^{(A)}+(1-\lambda) E_{\mathcal{F}_{1}}^{(B)} \geq E_{\mathcal{F}_{1}}^{\infty}\left(\lambda \rho_{A}+(1-\lambda) \rho_{B}\right) .
$$

Then, it is easy to show that

$$
E_{\mathcal{F}_{2}}^{\infty}\left(\rho_{C}\right) \leq E_{\mathcal{F}_{2}}^{\infty}\left(\lambda \rho_{A}+(1-\lambda) \rho_{B}\right) \leq \lambda E_{\mathcal{F}_{2}}^{(A)}+(1-\lambda) E_{\mathcal{F}_{2}}^{(B)},
$$

where the first inequality follows from Prop. 20, and the second one from the convexity of $E_{\mathcal{F}_{2}}^{\infty}$. Since $\rho_{C} \in \mathcal{F}_{\text {bank }}$, the point $P_{C}=\left(E_{\mathcal{F}_{1}}^{\infty}\left(\rho_{C}\right), E_{\mathcal{F}_{2}}^{\infty}\left(\rho_{C}\right)\right)$ is a point on the curve $\gamma_{\text {bank }}$.

It is easy to see that the above propositions imply that $c_{\text {bank }}$ is (strictly) monotonic decreasing, and convex. Since this function is defined on the closed interval $I_{1} \in \mathbb{R}$, we have that $c_{\text {bank }}$ is continuous

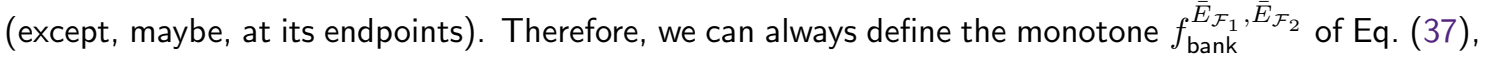
and it always satisfies condition B2. Finally, it is worth noticing that all the results apply if one (or both) the monotones are of the form of Eq. (31), since they satisfy all the necessary properties, in particular they are linear in both the tensor product and the admixture of states. 


\section{Energy-entanglement interconversion protocol}

In this section we provide a protocol, based on the compression theorems [90] known in quantum information theory, to perform interconversion of energy and entanglement using two batteries and a bank, see Sec. 5.2.1 for revising the set-up we use. In our protocol, we assume that the bank is initially described by $n \gg 1$ copies of a generic state $\rho \in \mathcal{S}_{\mathrm{p}_{0}}$, where $\mathrm{p}_{0}>\frac{1}{2}$, see Eq. (69), while the batteries $B_{W}$ and $B_{E}$ are initially in the states $\omega_{W}(k)$ and $\omega_{E}(h)$, respectively.

Our first step consists in using the allowed operation $\mathcal{E}_{\eta} \in \mathcal{C}_{\text {multi }}$, see Eq. (70), with $\eta=\left|\theta_{1}\right\rangle\left\langle\theta_{1}\right|$, to map the generic bank state $\rho$ into

$$
\rho_{\text {in }}=\mathrm{p}_{0}\left|\Psi_{\text {singlet }}\right\rangle\left\langle\Psi_{\text {singlet }}\left|+\left(1-\mathrm{p}_{0}\right)\right| \Psi_{\text {triplet }}^{(1)}\right\rangle\left\langle\Psi_{\text {triplet }}^{(1)}\right| .
$$

Thus, the bank system is now described by $n$ copies of the state $\rho_{\text {in }}$. Due to the central limit theorem, we can well approximate the state of the bank with an ensemble of its typical states, and in the following we will focus on the strongly typical ensemble,

$$
\Pi_{\text {st }}=\frac{1}{d_{\text {st }}} \sum_{i=1}^{d_{\text {st }}} \pi_{i}\left(\left|\Psi_{\text {singlet }}\right\rangle\left\langle\left.\Psi_{\text {singlet }}\right|^{\otimes n \mathrm{p}_{0}} \otimes \mid \Psi_{\text {triplet }}^{(1)}\right\rangle\left\langle\left.\Psi_{\text {triplet }}^{(1)}\right|^{\otimes n\left(1-\mathrm{p}_{0}\right)}\right),\right.
$$

where $d_{\mathrm{st}} \approx 2^{n \mathrm{~h}\left(\mathrm{p}_{0}\right)}$ is the number of states contained in the strongly typical set, the $\pi_{i}$ 's are the elements of the symmetric group acting on $n$ copies of the two-qubit system, and $\mathrm{h}(\cdot)$ is the binary entropy. Then, we can use a unitary operation to re-order the states in $\Pi_{\text {st }}$ so as to obtain

$$
\Pi_{\text {st }}^{\prime}=\sigma_{\mathrm{mm}}^{\otimes n \mathrm{~h}\left(\mathrm{p}_{0}\right)} \otimes\left|\Psi_{\text {singlet }}\right\rangle\left\langle\left.\Psi_{\text {singlet }}\right|^{\otimes n\left(1-\mathrm{h}\left(\mathrm{p}_{0}\right)\right)},\right.
$$

where $\sigma_{\mathrm{mm}}$ is the separable state introduced in Eq. (68). It is easy to see that this transformation, while leaving the amount of entanglement in the bank constant, $E_{\mathcal{F}_{\text {css }}}\left(\rho_{\mathrm{in}}^{\otimes n}\right)=E_{\mathcal{F}_{\text {css }}}\left(\Pi_{\mathrm{st}}^{\prime}\right)$, might not preserve the average energy. For this reason, while transforming the bank we also transform the energy battery, mapping $\omega_{W}(k)$ into $\omega_{W}(k+\Delta k)$ to keep the energy fixed.

We can now exchange some singlets with the entanglement battery. For example, we can perform a swap between the bank and the battery, moving in this way an integer number $r$ of singlets from the bank into the battery. This transformation maps the state of the bank into

$$
\Pi_{\mathrm{st}}^{\prime \prime}=\sigma_{\mathrm{mm}}^{\otimes n \mathrm{~h}\left(\mathrm{p}_{0}\right)+r} \otimes\left|\Psi_{\text {singlet }}\right\rangle\left\langle\left.\Psi_{\text {singlet }}\right|^{\otimes n\left(1-\mathrm{h}\left(\mathrm{p}_{0}\right)\right)-r},\right.
$$

and transforms the state of the entanglement battery from $\omega_{E}(h)$ into $\omega_{E}(h+r)$. Furthermore, the transformation also modify the energy of the bank, so that we need to map the state of the energy battery from $\omega_{W}(k+\Delta k)$ to $\omega_{W}\left(k+\Delta k^{\prime}\right)$. It is then possible to map the state $\Pi_{\text {st }}^{\prime \prime}$ into

$$
\Pi_{\text {st }}^{\prime \prime \prime}=\frac{1}{d_{\text {st }}^{\prime}} \sum_{i=1}^{d_{\text {st }}^{\prime}} \pi_{i}\left(\left|\Psi_{\text {singlet }}\right\rangle\left\langle\left.\Psi_{\text {singlet }}\right|^{\otimes n} \mathrm{p}_{0}^{\prime} \otimes \mid \Psi_{\text {triplet }}^{(1)}\right\rangle\left\langle\left.\Psi_{\text {triplet }}^{(1)}\right|^{\otimes n\left(1-\mathrm{p}_{0}^{\prime}\right)}\right),\right.
$$

where $\mathrm{p}_{0}^{\prime}$ is chosen in order to satisfy the equality

$$
n \mathrm{~h}\left(\mathrm{p}_{0}\right)+r=n \mathrm{~h}\left(\mathrm{p}_{0}^{\prime}\right),
$$

and $d_{\mathrm{st}}^{\prime}=2^{n \mathrm{~h}\left(\mathrm{p}_{0}^{\prime}\right)}$. The state $\Pi_{\mathrm{st}}^{\prime \prime \prime}$ is the strongly typical ensemble associated with $n$ copies of the state

$$
\rho_{\text {fin }}=\mathrm{p}_{0}^{\prime}\left|\Psi_{\text {singlet }}\right\rangle\left\langle\Psi_{\text {singlet }}\left|+\left(1-\mathrm{p}_{0}^{\prime}\right)\right| \Psi_{\text {triplet }}^{(1)}\right\rangle\left\langle\Psi_{\text {triplet }}^{(1)}\right|,
$$

where it is easy to show that the probability of occupation of the singlet is $\mathrm{p}_{0}^{\prime} \approx \mathrm{p}_{0}-\frac{r}{n} \frac{1}{\log \frac{\mathrm{p}_{0}}{1-\mathrm{p}_{0}}}$ for $n \gg 1$. The transformation mapping $\Pi_{\mathrm{st}}^{\prime \prime}$ into $\Pi_{\mathrm{st}}^{\prime \prime \prime}$ preserves the entanglement of the bank, while changing its energy. Therefore, while acting on the bank we have to modify the state of the energy battery as well, from $\omega_{W}\left(k+\Delta k^{\prime}\right)$ to $\omega_{W}\left(k+\Delta k^{\prime \prime}\right)$. In this way, we have modified the bank system by mapping $n$ copies of $\rho_{\text {in }}$ into $n$ copies of $\rho_{\text {fin }}$, and we kept entanglement and energy fixed on the global system by modifying the states of the batteries. Notice that the protocol can be extended to the 
typical ensembles by using a sub-linear ancillary system, and by considering corrections to the exchanged energy and entanglement of order $O(\sqrt{n})$.

During the protocol, the bank has exchanged $r$ singlets with the battery $B_{E}$, so that the gain in entanglement for this battery is

$$
\Delta W_{E}=E_{\mathcal{F}_{\text {css }}}\left(\omega_{E}(h+r)\right)-E_{\mathcal{F}_{\text {css }}}\left(\omega_{E}(h)\right)=r .
$$

In order to compute the amount of energy exchanged between the bank and the battery $B_{W}$, we consider the difference in average energy between $\rho_{\mathrm{in}}^{\otimes n}$ and $\rho_{\mathrm{fin}}^{\otimes n}$. In this way, we find that the amount of energy exchanged is

$$
\Delta W_{W}=M_{H}\left(\omega_{W}\left(k+\Delta k^{\prime \prime}\right)\right)-M_{H}\left(\omega_{W}(k)\right)=-\frac{\Delta E}{\log \frac{\mathrm{p}_{0}}{1-\mathrm{p}_{0}}} r,
$$

that is, energy has been paid in order to gain entanglement during the process. The interconversion relation between the two resources is given by

$$
\Delta W_{W}=-\frac{\Delta E}{\log \frac{\mathrm{p}_{0}}{1-\mathrm{p}_{0}}} \Delta W_{E},
$$

and we only need to show that the bank state has changed in a negligible way with respect to the related bank monotone. It is worth noting that, since the current theory satisfies all the properties we have considered in the main text, the bank monotone coincides, modulo a multiplicative constant, with the relative entropy distance from the set of states $\mathcal{S}_{\mathrm{p}_{0}}$ initially describing the bank.

Indeed, it is easy to show that the relative entropy distance from this set is given by a linear combination of the monotones $E_{\mathcal{F}_{\text {css }}}$ and $M_{H}$. For $\rho \in \mathcal{S}_{1}$ we find that

$$
E_{\mathcal{S}_{\mathrm{p}_{0}}}(\rho)=\inf _{\sigma \in \mathcal{S}_{\mathrm{p}_{0}}} D(\rho \| \sigma)=\left(E_{\mathcal{F}_{\text {css }}}(\rho)-\bar{E}_{\mathcal{F}_{\text {css }}}\right)+\frac{\log \frac{\mathrm{p}_{0}}{1-\mathrm{p}_{0}}}{\Delta E}\left(M_{H}(\rho)-\bar{M}_{H}\right),
$$

where we recall that $\bar{E}_{\mathcal{F}_{\text {css }}}=E_{\mathcal{F}_{\text {css }}}(\sigma)$ and $\bar{M}_{H}=M_{H}(\sigma)$, for any state $\sigma \in \mathcal{S}_{\mathrm{p}_{0}}$. The linear coefficient in the rhs of Eq. (121) is the (inverse) exchange rate that we find in the interconversion relation, Eq. (120). If we now consider the initial and final state of the bank, and we study how much the state is changed by the above protocol with respect to $E_{\mathcal{S}_{\mathrm{p}_{0}}}$, we find that

$$
E_{\mathcal{S}_{\mathrm{p}_{0}}}\left(\rho_{\mathrm{fin}}^{\otimes n}\right)-E_{\mathcal{S}_{\mathrm{p}_{0}}}\left(\rho_{\mathrm{in}}^{\otimes n}\right)=O\left(n^{-1}\right),
$$

so that, when $n \rightarrow \infty$, we obtain that the state of the bank is only infinitesimally changed, and can be used again to perform another resource interconversion with the same initial exchange rate.

\section{Proofs}

\section{D.1 Main results}

In the first part of this appendix we provide the proofs of the results presented in the main text. We start with the proof of the following theorem, where it is shown that a multi-resource theory which satisfies the asymptotic equivalence property of Def. 1 has a unique quantifier for each of the resources present in the theory. This theorem is introduced in Sec. 3.3.

Theorem 5. Consider the resource theory $R_{\text {multi }}$ with $m$ resources, equipped with the batteries $B_{i}$ 's, where $i=1, \ldots, m$. Suppose the theory satisfies the asymptotic equivalence property with respect to the set of monotones $\left\{f_{i}\right\}_{i=1}^{m}$. If these monotones satisfy the properties $M 1-M 7$, then the amount of $i$-th resource contained in the main system $S$ is uniquely quantified by the regularisation of the monotone $f_{i}$ (modulo a multiplicative constant).

Proof. Let us prove that $f_{1}^{\infty}$ uniquely quantifies the amount of 1 -st resource contained in the main system (the proof for the other $f_{i \neq 1}$ 's is analogous). We prove the theorem by contradiction. Suppose that there exists two monotones $f_{1}$ and $g_{1}$ satisfying the properties $\mathrm{M} 1-\mathrm{M} 7$, such that 
1. $\exists \rho \in \mathcal{S}\left(\mathcal{H}_{S}\right)$, where $\rho \notin \mathcal{F}_{1}$, for which $f_{1}^{\infty}(\rho)=g_{1}^{\infty}(\rho)$ (this is always possible by rescaling the monotone $g$ ).

2. $\exists \sigma \in \mathcal{S}\left(\mathcal{H}_{S}\right)$, where $\sigma \notin \mathcal{F}_{1}$, for which $f_{1}^{\infty}(\sigma) \neq g_{1}^{\infty}(\sigma)$ (that is, $f_{1}$ is not unique).

Consider now the values of $f_{1}^{\infty}(\rho)$ and $f_{1}^{\infty}(\sigma)$. If these are equal, it is easy to see, using the asymptotic equivalence property, that $f_{1}$ is unique. Suppose instead that they are not equal. Then, there exists $n, k \in \mathbb{N}^{9}$ such that

$$
n f_{1}^{\infty}(\rho)=k f_{1}^{\infty}(\sigma) .
$$

Let us consider the system together with the batteries $B_{i}$ 's, initially in the state $\rho^{\otimes n} \otimes \omega_{1} \otimes \ldots \otimes \omega_{m}$. Then, we take the states $\omega_{i}^{\prime} \in \mathcal{S}\left(\mathcal{H}_{B_{i}}\right)$, where $i=1, \ldots, m$, such that

$$
\begin{aligned}
f_{i}^{\infty}\left(\rho^{\otimes n} \otimes \omega_{1} \otimes \ldots \otimes \omega_{m}\right) & =f_{i}^{\infty}\left(\gamma_{n} \otimes \omega_{1}^{\prime} \otimes \ldots \otimes \omega_{m}^{\prime}\right), \forall i \in\{1, \ldots, m\}, \\
f_{j}^{\infty}\left(\omega_{i}\right) & =f_{j}^{\infty}\left(\omega_{i}^{\prime}\right), \forall i, j \in\{1, \ldots, m\}, i \neq j,
\end{aligned}
$$

where $\gamma_{n} \in \mathcal{F}_{1}^{(n)}$. Due to the asymptotic equivalence property, the conditions in Eq. (124) imply that there exists a sequence of maps $\left\{\tilde{\mathcal{E}}_{N}\right\}_{N}$ of the form of Eq. (13) such that

$$
\lim _{N \rightarrow \infty}\left\|\tilde{\mathcal{E}}_{N}\left(\left(\rho^{\otimes n} \otimes \omega_{1} \otimes \ldots \otimes \omega_{m}\right)^{\otimes N}\right)-\left(\gamma_{n} \otimes \omega_{1}^{\prime} \otimes \ldots \otimes \omega_{m}^{\prime}\right)^{\otimes N}\right\|_{1}=0
$$

as well as another sequence of maps performing the reverse transformation. From the asymptotic continuity of $g_{1}$, property M7, it then follows that

$$
g_{1}\left(\tilde{\mathcal{E}}_{N}\left(\left(\rho^{\otimes n} \otimes \omega_{1} \otimes \ldots \otimes \omega_{m}\right)^{\otimes N}\right)\right)=g_{1}\left(\left(\gamma_{n} \otimes \omega_{1}^{\prime} \otimes \ldots \otimes \omega_{m}^{\prime}\right)^{\otimes N}\right)+o(N) .
$$

Let us consider the lhs of the above equation, and recall that the map $\tilde{\mathcal{E}}_{N}$ is obtained by applying an allowed operation to $N$ copies of the system together with a sub-linear ancilla $\eta_{N}^{(A)}$, see Eq. (13). For simplicity, let us refer to $\rho^{\otimes n} \otimes \omega_{1} \otimes \ldots \otimes \omega_{m}$ as $\Omega$ in the following chain of inequalities,

$$
\begin{aligned}
g_{1}\left(\tilde{\mathcal{E}}_{N}\left(\Omega^{\otimes N}\right)\right) & =g_{1}\left(\operatorname{Tr}_{A}\left[\mathcal{E}_{N}\left(\Omega^{\otimes N} \otimes \eta_{N}^{(A)}\right)\right]\right) \leq g_{1}\left(\mathcal{E}_{N}\left(\Omega^{\otimes N} \otimes \eta_{N}^{(A)}\right)\right) \leq g_{1}\left(\Omega^{\otimes N} \otimes \eta_{N}^{(A)}\right) \\
& \leq g_{1}\left(\Omega^{\otimes N}\right)+g_{1}\left(\eta_{N}^{(A)}\right) \leq g_{1}\left(\Omega^{\otimes N}\right)+o(N)
\end{aligned}
$$

where the first inequality follows from property M4, the second one from the monotonicity of $g_{1}$ under allowed operations, the third one from the sub-additivity of $g_{1}$, property $\mathrm{M} 5$, and the last inequality from property M6 and the fact that the ancilla is sub-linear in $N$. If we now combine this equation with the previous one, we divide both sides by $N$, and we send it to infinity, we obtain that the regularised version of $g_{1}$ is such that,

$$
g_{1}^{\infty}\left(\rho^{\otimes n} \otimes \omega_{1} \otimes \ldots \otimes \omega_{m}\right) \geq g_{1}^{\infty}\left(\gamma_{n} \otimes \omega_{1}^{\prime} \otimes \ldots \otimes \omega_{m}^{\prime}\right) .
$$

By using the same argument for the sequence of maps performing the reverse transformation, we find that the above equation needs to hold as an equality, that is,

$$
g_{1}^{\infty}\left(\rho^{\otimes n} \otimes \omega_{1} \otimes \ldots \otimes \omega_{m}\right)=g_{1}^{\infty}\left(\gamma_{n} \otimes \omega_{1}^{\prime} \otimes \ldots \otimes \omega_{m}^{\prime}\right) .
$$

We can now separate each contribution to $g_{1}$ thanks to the property M2, use the fact that the batteries $B_{i \neq 1}$ 's are not changing their value of $g_{1}$, property $\mathrm{M} 1$, and the fact that the final state of the system does not contain any resource associated with $g_{1}$, property M3. Then, we find that

$$
n g_{1}^{\infty}(\rho)=g_{1}^{\infty}\left(\omega_{1}^{\prime}\right)-g_{1}^{\infty}\left(\omega_{1}\right),
$$

where we have also used Lem. 17. The same result follows for $f_{1}$, so that we find that

$$
n f_{1}^{\infty}(\rho)=f_{1}^{\infty}\left(\omega_{1}^{\prime}\right)-f_{1}^{\infty}\left(\omega_{1}\right) .
$$

${ }^{9}$ Where we assume that all physically meaningful values of the $f_{i}^{\infty}$ 's are in $\mathbb{Q}$, which we recall is dense in $\mathbb{R}$. 
If we now consider Eqs. (123) and (132), we find that

$$
k f_{1}^{\infty}(\sigma)=f_{1}^{\infty}\left(\omega_{1}^{\prime}\right)-f_{1}^{\infty}\left(\omega_{1}\right) .
$$

We can add to the above equation the term $f_{1}^{\infty}\left(\gamma_{k}\right)$, where $\gamma_{k} \in \mathcal{F}_{1}^{(k)}$, since this term is equal to zero due to property M3. Then, we find

$$
k f_{1}^{\infty}(\sigma)+f_{1}^{\infty}\left(\omega_{1}\right)=f_{1}^{\infty}\left(\gamma_{k}\right)+f_{1}^{\infty}\left(\omega_{1}^{\prime}\right) .
$$

Now, we want to introduce the initial and final states of the batteries $B_{i \neq 1}$ 's, so as to be sure that the transformation from $\sigma^{\otimes k}$ into $\gamma_{k}$ does not violate the conservation of the other resources. Specifically, we introduce $\omega_{i}, \omega_{i}^{\prime \prime} \in \mathcal{S}\left(\mathcal{H}_{B_{i}}\right)$ for $i \neq 1$, such that

$$
\begin{aligned}
f_{i}^{\infty}\left(\sigma^{\otimes k} \otimes \omega_{1} \otimes \omega_{2} \otimes \ldots \otimes \omega_{m}\right) & =f_{i}^{\infty}\left(\gamma_{k} \otimes \omega_{1}^{\prime} \otimes \omega_{2}^{\prime \prime} \otimes \ldots \otimes \omega_{m}^{\prime \prime}\right), \forall i \in\{2, \ldots, m\}, \\
f_{1}^{\infty}\left(\omega_{i}\right) & =f_{1}^{\infty}\left(\omega_{i}^{\prime \prime}\right), \forall i \in\{2, \ldots, m\}, \\
f_{j}^{\infty}\left(\omega_{i}\right) & =f_{j}^{\infty}\left(\omega_{i}^{\prime \prime}\right), \forall i, j \in\{2, \ldots, m\}, i \neq j .
\end{aligned}
$$

Then, using the constraints of Eq. (136) over the states of the $B_{i \neq 1}$ 's batteries, we can re-write Eq. (134) as

$$
k f_{1}^{\infty}(\sigma)+f_{1}^{\infty}\left(\omega_{1}\right)+f_{1}^{\infty}\left(\omega_{2}\right)+\ldots+f_{1}^{\infty}\left(\omega_{m}\right)=f_{1}^{\infty}\left(\gamma_{k}\right)+f_{1}^{\infty}\left(\omega_{1}^{\prime}\right)+f_{1}^{\infty}\left(\omega_{2}^{\prime \prime}\right)+\ldots+f_{1}^{\infty}\left(\omega_{m}^{\prime \prime}\right) .
$$

If we now use Lem. 17 and property M1, we find that

$$
f_{1}^{\infty}\left(\sigma^{\otimes k} \otimes \omega_{1} \otimes \omega_{2} \otimes \ldots \otimes \omega_{m}\right)=f_{1}^{\infty}\left(\gamma_{k} \otimes \omega_{1}^{\prime} \otimes \omega_{2}^{\prime \prime} \otimes \ldots \otimes \omega_{m}^{\prime \prime}\right)
$$

From Eqs. (135) and (139) it follows, using the asymptotic equivalence property, that there exists a sequence of maps $\left\{\tilde{\mathcal{E}}_{N}^{\prime}\right\}_{N}$ such that

$$
\lim _{N \rightarrow \infty}\left\|\tilde{\mathcal{E}}_{N}^{\prime}\left(\left(\sigma^{\otimes k} \otimes \omega_{1} \otimes \omega_{2} \otimes \ldots \otimes \omega_{m}\right)^{\otimes N}\right)-\left(\gamma_{k} \otimes \omega_{1}^{\prime} \otimes \omega_{2}^{\prime \prime} \otimes \ldots \otimes \omega_{m}^{\prime \prime}\right)^{\otimes N}\right\|_{1}=0,
$$

as well as a related sequence of maps performing the reverse transformation. Using the properties of $g_{1}$, as we did before, we find that

$$
k g_{1}^{\infty}(\sigma)=g_{1}^{\infty}\left(\omega_{1}^{\prime}\right)-g_{1}^{\infty}\left(\omega_{1}\right) .
$$

Then, combining Eqs. (131) and (141), we obtain that

$$
n g_{1}^{\infty}(\rho)=k g_{1}^{\infty}(\sigma) .
$$

Finally, using Eq. (123) and the initial assumption on the state $\rho$, we find that

$$
f_{1}^{\infty}(\sigma)=g_{1}^{\infty}(\sigma),
$$

which contradicts our initial assumption. Therefore, $f_{1}^{\infty}$ uniquely quantify the amount of 1-st resource contained in the main system.

In the next theorem, first stated in Sec. 4.1, we show that in the presence of a bank two resources can always be exchanged one for the other, while the state of the bank is only infinitesimally modified by the resource interconversion.

Theorem 9. Consider a resource theory $R_{\text {multi }}$ with two resources, equipped with the batteries $B_{1}$ and $B_{2}$. Suppose the theory satisfies asymptotic equivalence with respect to the monotones $E_{\mathcal{F}_{1}}$ and $E_{\mathcal{F}_{2}}$, i.e. the relative entropy distances from the invariant sets of the theory, and that these sets satisfy the properties F1, F2, F3, and F5b. Then, the resource interconversion of Eq. (40), where the bank has to transform in accord to condition $\mathrm{X} 1$, is solely regulated by the following relation,

$$
\alpha \Delta W_{1}=-\beta \Delta W_{2}+\delta_{n} .
$$

Furthermore, when the number of copies of the bank system $n$ is sent to infinity, we have that the above equation reduces to the following one, which we refer to as the interconversion relation,

$$
\Delta W_{1}=-\frac{\beta}{\alpha} \Delta W_{2},
$$

where the amount of resources exchanged $\Delta W_{i}$ is non-zero. 
Proof. Let us consider the resource interconversion of Eq. (40), where a global operation is performed over bank and batteries, and the sole constraint over the bank system is given by condition X1. As we discussed in Sec. 3.2, in order for the transformation to happen, the conditions of Eq. (21) need to be satisfied for both monotones $E_{\mathcal{F}_{1}}$ and $E_{\mathcal{F}_{2}}$, which in particular implies that the amount of resources exchanged with the batteries is

$$
\Delta W_{i}=n\left(E_{\mathcal{F}_{i}}(\rho)-E_{\mathcal{F}_{i}}(\tilde{\rho})\right), \quad i=1,2,
$$

where we have used property F5b. Furthermore, since $f_{\text {bank }}^{\bar{E}_{\mathcal{F}_{1}}, \bar{E}_{\mathcal{F}_{2}}}$ is monotonic under the set of allowed operations, property $\mathrm{B} 7$, we find that

$$
f_{\text {bank }}^{\bar{E}_{\mathcal{F}_{1}}, \bar{E}_{\mathcal{F}_{2}}}\left(\rho^{\otimes n} \otimes \omega_{1} \otimes \omega_{2}\right)=f_{\text {bank }}^{\bar{E}_{\mathcal{F}_{1}}, \bar{E}_{\mathcal{F}_{2}}}\left(\tilde{\rho}^{\otimes n} \otimes \omega_{1}^{\prime} \otimes \omega_{2}^{\prime}\right) .
$$

Then, since the global system is given by many copies of $\mathcal{H}$, and since the bank monotone is additive, property B3, we can separate the contribution given by bank and batteries. Furthermore, from the definition of bank monotone, Eq. (37), and the main property of the batteries, condition M1, it follows that

$\alpha\left(E_{\mathcal{F}_{1}}\left(\rho^{\otimes n}\right)+E_{\mathcal{F}_{1}}\left(\omega_{1}\right)\right)+\beta\left(E_{\mathcal{F}_{2}}\left(\rho^{\otimes n}\right)+E_{\mathcal{F}_{2}}\left(\omega_{2}\right)\right)=\alpha\left(E_{\mathcal{F}_{1}}\left(\tilde{\rho}^{\otimes n}\right)+E_{\mathcal{F}_{1}}\left(\omega_{1}^{\prime}\right)\right)+\beta\left(E_{\mathcal{F}_{2}}\left(\tilde{\rho}^{\otimes n}\right)+E_{\mathcal{F}_{2}}\left(\omega_{2}^{\prime}\right)\right)$.

Now, if we re-order the terms in the above equation, and we use Eq. (37) again, we obtain

$$
f_{\text {bank }}^{\bar{E}_{\mathcal{F}_{1}}, \bar{E}_{\mathcal{F}_{2}}}\left(\rho^{\otimes n}\right)-f_{\text {bank }}^{\bar{E}_{\mathcal{F}_{1}}, \bar{E}_{\mathcal{F}_{2}}}\left(\tilde{\rho}^{\otimes n}\right)=\alpha\left(E_{\mathcal{F}_{1}}\left(\omega_{1}^{\prime}\right)-E_{\mathcal{F}_{1}}\left(\omega_{1}\right)\right)+\beta\left(E_{\mathcal{F}_{2}}\left(\omega_{2}^{\prime}\right)-E_{\mathcal{F}_{2}}\left(\omega_{2}\right)\right) .
$$

If we use property $\mathrm{X} 1$ together with the definitions of $\Delta W_{1}$ and $\Delta W_{2}$ given in Eq. (23), we get that

$$
\alpha \Delta W_{1}=-\beta \Delta W_{2}+\delta_{n},
$$

where $\delta_{n} \rightarrow 0$ as $n$ tends to infinity. However, we are still left to show that, when $n \rightarrow \infty$, the amount of resources exchanged by the batteries remains finite.

Let us first recall that the way in which the monotone $f_{\text {bank }}^{\bar{E}_{\mathcal{F}_{1}}, \bar{E}_{\mathcal{F}_{2}}}$ is built implies that this monotone is tangent to the state-space, see property B2 and Fig. 5. As a result, we have that the curve of bank states, see Eq. (104) in appendix B, can be approximate, in the neighbourhood of $\mathcal{F}_{\text {bank }}\left(\bar{E}_{\mathcal{F}_{1}}, \bar{E}_{\mathcal{F}_{2}}\right)$, by a line. This implies that, if we take the state $\tilde{\rho}$ in the set of bank states $\mathcal{F}_{\text {bank, }}$ such that

$$
E_{\mathcal{F}_{1}}(\tilde{\rho})=E_{\mathcal{F}_{1}}(\rho)-\epsilon,
$$

where we recall $\rho \in \mathcal{F}_{\text {bank }}\left(\bar{E}_{\mathcal{F}_{1}}, \bar{E}_{\mathcal{F}_{2}}\right)$, and $\epsilon \ll 1$, we find that the value of the monotone $E_{\mathcal{F}_{2}}$ for this state is

$$
E_{\mathcal{F}_{1}}(\tilde{\rho})=E_{\mathcal{F}_{2}}(\rho)+\frac{\alpha}{\beta} \epsilon+O\left(\epsilon^{2}\right) .
$$

Then, it is easy to see that, if we map $\rho$ into $\tilde{\rho}$ during the resource interconversion, we obtain the following

$$
\Delta W_{1}=n \epsilon \quad, \quad \Delta W_{2}=-n \frac{\alpha}{\beta} \epsilon+O\left(n \epsilon^{2}\right) \quad, \quad \delta_{n}=O\left(n \epsilon^{2}\right),
$$

where the first two equations follow from Eq. (144), while the last one is given by Eq. (41). Thus, if we take $\epsilon \propto \frac{1}{n}$, and we send $n$ to infinity, we get that the amount of resources $\Delta W_{i}$ exchanged during the transformations are finite and their value is arbitrary, while the change in the bank monotone over the bank system $\delta_{n}$ is infinitesimal.

The next theorem can be found in Sec. 4.2. The theorem states that, given a multi-resource theory with a non-empty set of bank states, we can always build a single-resource theory out of it, by extending the class of allowed operations with the possibility of adding ancillary systems described by the bank states, see Def. 10. In particular, we show that if the multi-resource theory satisfies the asymptotic equivalence property, so does the single-resource theory with respect to the bank monotone of Eq. (37). 
Theorem 11. Consider the two-resource theory $R_{\text {multi }}$ with allowed operations $\mathcal{C}_{\text {multi, }}$ and invariant sets $\mathcal{F}_{1}$ and $\mathcal{F}_{2}$ which satisfy the properties $F 1, F 2, F 3$, and $F 5 b$. Suppose the theory satisfies the asymptotic equivalence property with respect to the monotones $E_{\mathcal{F}_{1}}$ and $E_{\mathcal{F}_{2}}$. Then, given the subset of bank states $\mathcal{F}_{\text {bank }}\left(\bar{E}_{\mathcal{F}_{1}}, \bar{E}_{\mathcal{F}_{2}}\right)$, the single-resource theory $R_{\text {single }}$ with allowed operations $\mathcal{C}_{\text {single }}$ satisfies the asymptotic equivalence property with respect to $f_{\text {bank }}^{\bar{E}_{\mathcal{F}_{1}}, \bar{E}_{\mathcal{F}_{2}}}$.

Proof. (a) We start the proof by showing that, for the single resource theory $R_{\text {single, }}$, the second statement in Def. 1 implies the first one. In other words, we want to show that for any two states $\rho, \sigma \in \mathcal{S}(\mathcal{H})$ which can be asymptotically mapped into one another with the allowed operations $\mathcal{C}_{\text {single }}$, the value of the bank monotone on the two states is the same. Suppose there exists a sequence of operations $\left\{\tilde{\mathcal{E}}_{N}^{(\mathrm{s})}\right\}_{N}$ such that $\lim _{N \rightarrow \infty}\left\|\tilde{\mathcal{E}}_{N}^{(\mathrm{s})}\left(\rho^{\otimes N}\right)-\sigma^{\otimes N}\right\|_{1}=0$, where these maps are of the form

$$
\tilde{\mathcal{E}}_{N}^{(\mathrm{s})}(\cdot)=\operatorname{Tr}_{A}\left[\mathcal{E}_{N}^{(\mathrm{s})}\left(\cdot \otimes \eta_{N}^{(A)}\right)\right],
$$

with $\eta_{N}^{(A)} \in \mathcal{S}\left(\mathcal{H}^{\otimes o(N)}\right)$ an arbitrary state of a sub-linear ancilla, and $\mathcal{E}_{N}^{(\mathrm{s})}$ an allowed operation for $\mathrm{R}_{\text {single. }}$. Likewise, suppose there is a sequence of maps that perform the reverse transformation. If we use the asymptotic continuity of the bank monotone, property B6, it follows that

$$
f_{\text {bank }}^{\bar{E}_{\mathcal{F}_{1}}, \bar{E}_{\mathcal{F}_{2}}}\left(\tilde{\mathcal{E}}_{N}^{(\mathrm{s})}\left(\rho^{\otimes N}\right)\right)=f_{\text {bank }}^{\bar{E}_{\mathcal{F}_{1}}, \bar{E}_{\mathcal{F}_{2}}}\left(\sigma^{\otimes N}\right)+o(N) .
$$

Then, by using the properties B1 - B7 of the bank monotone, we can prove the following chain of inequalities for the lhs of the above equation

$$
\begin{aligned}
f_{\text {bank }}^{\bar{E}_{\mathcal{F}_{1}}, \bar{E}_{\mathcal{F}_{2}}}\left(\tilde{\mathcal{E}}_{N}^{(\mathrm{s})}\left(\rho^{\otimes N}\right)\right) & =f_{\text {bank }}^{\bar{E}_{\mathcal{F}_{1}}, \bar{E}_{\mathcal{F}_{2}}}\left(\operatorname{Tr}_{A}\left[\mathcal{E}_{N}^{(\mathrm{s})}\left(\rho^{\otimes N} \otimes \eta_{N}^{(A)}\right)\right]\right) \leq f_{\text {bank }}^{\bar{E}_{\mathcal{F}_{1}}, \bar{E}_{\mathcal{F}_{2}}}\left(\mathcal{E}_{N}^{(\mathrm{s})}\left(\rho^{\otimes N} \otimes \eta_{N}^{(A)}\right)\right) \\
& \leq f_{\text {bank }}^{\bar{E}_{\mathcal{F}_{1}}, \bar{E}_{\mathcal{F}_{2}}}\left(\rho^{\otimes N} \otimes \eta_{N}^{(A)}\right)=f_{\text {bank }}^{\bar{E}_{\mathcal{F}_{1}}, \bar{E}_{\mathcal{F}_{2}}}\left(\rho^{\otimes N}\right)+f_{\text {bank }}^{\bar{E}_{\mathcal{F}_{1}}, \bar{E}_{\mathcal{F}_{2}}}\left(\eta_{N}^{(A)}\right) \\
& \leq f_{\text {bank }}^{\bar{E}_{\mathcal{F}_{1}}, \bar{E}_{\mathcal{F}_{2}}}\left(\rho^{\otimes N}\right)+o(N)
\end{aligned}
$$

where the first inequality follows from monotonicity under partial trace, property B4, the second one from monotonicity under the allowed operations $\mathcal{C}_{\text {single }}$ (that we still need to show), the equality follows from additivity, property B3, and the last inequality from the sub-extensivity of the monotone, property B5. If we use the same argument for the sequence of maps performing the reverse transformation, and we regularise the monotones by dividing the equations by the number of copies $N$, and sending $N$ to infinity, we find that

$$
f_{\text {bank }}^{\bar{E}_{\mathcal{F}_{1}}, \bar{E}_{\mathcal{F}_{2}}}(\rho)=f_{\text {bank }}^{\bar{E}_{\mathcal{F}_{1}}, \bar{E}_{\mathcal{F}_{2}}}(\sigma),
$$

which proves the asymptotic equivalence property in one direction.

We still need to show that the bank monotone is monotonic under the allowed operations $\mathcal{C}_{\text {single }}$ of the single-resource theory. Recall that the most general of these operations, Eq. (44), is given by

$$
\mathcal{E}^{(\mathrm{s})}(\rho)=\operatorname{Tr}_{P^{(n)}}\left[\mathcal{E}\left(\rho \otimes \rho_{P}^{\otimes n}\right)\right],
$$

where $\mathcal{E} \in \mathcal{C}_{\text {multit }}$, and we add $n \in \mathbb{N}$ copies of the bank state $\rho_{P} \in \mathcal{F}_{\text {bank }}\left(\bar{E}_{\mathcal{F}_{1}}, \bar{E}_{\mathcal{F}_{2}}\right)$. Then, using the properties of the bank monotone, we can show that

$$
\begin{aligned}
& f_{\text {bank }}^{\bar{E}_{\mathcal{F}_{1}}, \bar{E}_{\mathcal{F}_{2}}}\left(\mathcal{E}^{(\mathrm{s})}(\rho)\right)=f_{\text {bank }}^{\bar{E}_{\mathcal{F}_{1}}, \bar{E}_{\mathcal{F}_{2}}}\left(\operatorname{Tr}_{P^{(n)}}\left[\mathcal{E}\left(\rho \otimes \rho_{P}^{\otimes n}\right)\right]\right) \leq f_{\text {bank }}^{\bar{E}_{\mathcal{F}_{1}}, \bar{E}_{\mathcal{F}_{2}}}\left(\mathcal{E}\left(\rho \otimes \rho_{P}^{\otimes n}\right)\right) \\
& \leq f_{\text {bank }}^{\bar{E}_{\mathcal{F}_{1}}, \bar{E}_{\mathcal{F}_{2}}}\left(\rho \otimes \rho_{P}^{\otimes n}\right)=f_{\text {bank }}^{\bar{E}_{\mathcal{F}_{1}}, \bar{E}_{\mathcal{F}_{2}}}(\rho)+f_{\text {bank }}^{\bar{E}_{\mathcal{F}_{1}}, \bar{E}_{\mathcal{F}_{2}}}\left(\rho_{P}^{\otimes n}\right) \\
& =f_{\text {bank }}^{\bar{E}_{\mathcal{F}_{1}}, \bar{E}_{\mathcal{F}_{2}}}(\rho),
\end{aligned}
$$

where the first inequality follows from property $\mathrm{B} 4$, the second one from the monotonicity under the allowed operations $\mathcal{C}_{\text {multi }}$, property $\mathrm{B} 7$, and the last two equalities from additivity, property $\mathrm{B} 3$, and the fact that the bank monotone is equal to zero over the bank states, property B1, respectively.

(b) We now want to prove the other direction of the asymptotic equivalence property for the resource theory $\mathrm{R}_{\text {single }}$, i.e., that the first statement in Def. 1 implies the second one. In other words, 
we want to show that for all states $\rho, \sigma \in \mathcal{S}(\mathcal{H})$ such that $f_{\mathrm{bank}}^{\bar{E}_{\mathcal{F}_{1}}, \bar{E}_{\mathcal{F}_{2}}}(\rho)=f_{\text {bank }}^{\bar{E}_{\mathcal{F}_{1}}, \bar{E}_{\mathcal{F}_{2}}}(\sigma)$, there exists a sequence of operations $\left\{\tilde{\mathcal{E}}_{N}^{(s)}\right\}_{N}$ of the form given in Eq. (152), mapping $N$ copies of $\rho$ into $N$ copies of $\sigma$, where $N \rightarrow \infty$. Before proving this part of the theorem, we recall that, given the bank state $\rho_{P} \in \mathcal{F}_{\text {bank }}\left(\bar{E}_{\mathcal{F}_{1}}, \bar{E}_{\mathcal{F}_{2}}\right)$, all other bank states $\tilde{\rho}_{P} \in \mathcal{F}_{\text {bank }}$ are such that, if $E_{\mathcal{F}_{1}}\left(\tilde{\rho}_{P}\right)=E_{\mathcal{F}_{1}}\left(\rho_{P}\right)+\delta$ with $\delta \ll 1$, then

$$
E_{\mathcal{F}_{2}}\left(\tilde{\rho}_{P}\right)=E_{\mathcal{F}_{2}}\left(\rho_{P}\right)-\frac{\alpha}{\beta} \delta+O\left(\delta^{2}\right)
$$

which follows from the fact that $f_{\text {bank }}^{\bar{E}_{\mathcal{F}_{1}}, \bar{E}_{\mathcal{F}_{2}}}=0$ parametrises the line which is tangent to the state space and passes through the point $\left(\bar{E}_{\mathcal{F}_{1}}, E_{\mathcal{F}_{2}}\right)$, see appendix $\mathrm{B}$.

Given the two states $\rho, \sigma \in \mathcal{S}(\mathcal{H})$ with same value of the monotone $f_{\text {bank }}^{\bar{E}_{\mathcal{F}_{1}}, \bar{E}_{\mathcal{F}_{2}}}$, let us introduce the sequences of states $\left\{\sigma_{n} \in \mathcal{S}(\mathcal{H})\right\}_{n}$ and $\left\{\tilde{\rho}_{P, n} \in \mathcal{F}_{\text {bank }}\right\}_{n}$ such that, for $n \in \mathbb{N}$ big enough, we have

$$
\begin{aligned}
E_{\mathcal{F}_{1}}\left(\sigma_{n}\right) & =E_{\mathcal{F}_{1}}(\sigma) \\
E_{\mathcal{F}_{1}}\left(\rho \otimes \rho_{P}^{\otimes n}\right) & =E_{\mathcal{F}_{1}}\left(\sigma_{n} \otimes\left(\tilde{\rho}_{P, n}\right)^{\otimes n}\right), \\
E_{\mathcal{F}_{2}}\left(\rho \otimes \rho_{P}^{\otimes n}\right) & =E_{\mathcal{F}_{2}}\left(\sigma_{n} \otimes\left(\tilde{\rho}_{P, n}\right)^{\otimes n}\right),
\end{aligned}
$$

where $\rho_{P} \in \mathcal{F}_{\text {bank }}\left(\bar{E}_{\mathcal{F}_{1}}, \bar{E}_{\mathcal{F}_{2}}\right)$. From the above equations, and from the additivity of $E_{\mathcal{F}_{1}}$, which follows from property $\mathrm{F} 5 \mathrm{~b}$, we obtain that

$$
E_{\mathcal{F}_{1}}\left(\tilde{\rho}_{P, n}\right)=E_{\mathcal{F}_{1}}\left(\rho_{P}\right)+\frac{1}{n}\left(E_{\mathcal{F}_{1}}(\rho)-E_{\mathcal{F}_{1}}(\sigma)\right) .
$$

Notice that, for $n \rightarrow \infty$, we have that $\frac{1}{n}\left(E_{\mathcal{F}_{1}}(\rho)-E_{\mathcal{F}_{1}}(\sigma)\right) \rightarrow 0$, and therefore, for $n$ sufficiently big, it follows from Eq. (158) that

$$
E_{\mathcal{F}_{2}}\left(\tilde{\rho}_{P, n}\right)=E_{\mathcal{F}_{2}}\left(\rho_{P}\right)-\frac{\alpha}{\beta} \frac{1}{n}\left(E_{\mathcal{F}_{1}}(\rho)-E_{\mathcal{F}_{1}}(\sigma)\right)+O\left(n^{-2}\right) .
$$

If we now combine Eq. (161) and (163) together, we use the additivity of $E_{\mathcal{F}_{2}}$, and we use the fact that $\rho$ and $\sigma$ have the same value of the bank monotone, we obtain the following

$$
E_{\mathcal{F}_{2}}\left(\sigma_{n}\right)=E_{\mathcal{F}_{2}}(\sigma)+O\left(n^{-1}\right) \text {. }
$$

Let us now focus on the operations mapping $\rho$ into $\sigma$. We do this in two steps. First, we use the fact that the theory $\mathrm{R}_{\text {multi }}$ satisfies asymptotic equivalence, and we consider the Eqs. (160) and (161). These equations imply that, for all $n \in \mathbb{N}$, there exists of a sequence of maps $\left\{\tilde{\mathcal{E}}_{N, n}\right\}_{N}$ such that

$$
\lim _{N \rightarrow \infty}\left\|\tilde{\mathcal{E}}_{N, n}\left(\left(\rho \otimes \rho_{P}^{\otimes n}\right)^{\otimes N}\right)-\left(\sigma_{n} \otimes\left(\tilde{\rho}_{P, n}\right)^{\otimes n}\right)^{\otimes N}\right\|_{1}=0 .
$$

As per definition of asymptotic equivalence, the maps $\tilde{\mathcal{E}}_{N, n}: \mathcal{S}\left(\mathcal{H}^{\otimes N(n+1)}\right) \rightarrow \mathcal{S}\left(\mathcal{H}^{\otimes N(n+1)}\right)$ are of the form

$$
\tilde{\mathcal{E}}_{N, n}(\cdot)=\operatorname{Tr}_{A}\left[\mathcal{E}_{N, n}\left(\cdot \otimes \eta_{N}^{(A)}\right)\right]
$$

where the map $\mathcal{E}_{N, n}$ is an allowed operation of $\mathrm{R}_{\text {multi }}$ acting on system and ancilla, and the state of the ancilla is $\eta_{N}^{(A)} \in \mathcal{S}\left(\left(\mathcal{H}^{\otimes n+1}\right)^{\otimes f(N)}\right)$, where $f(N)=o(N)$. Notice that, in particular, we can take $n$ to be a monotonic function of $N, n=g(N)$, such that $\lim _{N \rightarrow \infty} g(N)=\infty$ and $f(N) g(N)=o(N)$. For example, if $f(N) \propto N^{1 / 2}$, we can chose $g(N) \propto N^{1 / 4}$, so that their product is $N^{3 / 4}=o(N)$.

We can now define the sequence of maps $\left\{\tilde{\mathcal{E}}_{N}^{(\mathrm{s})}\right\}_{N}$ acting on $\mathcal{S}\left(\mathcal{H}^{\otimes N}\right)$. These maps are defined as

$$
\tilde{\mathcal{E}}_{N}^{(\mathrm{s})}\left(\rho^{\otimes N}\right)=\operatorname{Tr}_{P}\left[\tilde{\mathcal{E}}_{N, g(N)}\left(\rho^{\otimes N} \otimes \rho_{P}^{\otimes N g(N)}\right)\right]
$$

where we are tracing out the part of the system which was initially in the state $\rho_{P}^{\otimes N g(N)}$. It is interesting to notice that this system is super-linear in the number of copies $N$ of $\rho$, a condition that seems to be 
necessary to achieve the conversion, see Ref. [10] for an example in thermodynamics. We can re-write these maps as

$$
\tilde{\mathcal{E}}_{N}^{(\mathrm{s})}\left(\rho^{\otimes N}\right)=\operatorname{Tr}_{A}\left[\mathcal{E}_{N}^{(\mathrm{s})}\left(\rho^{\otimes N} \otimes \eta_{N}^{(A)}\right)\right],
$$

where we recall that the ancillary system still lives on a sub-linear number of copies of $\mathcal{H}$, due to our choice of the function $g(N)$, and the operation $\mathcal{E}_{N}^{(\mathrm{s})}$ is an allowed operations for the theory $\mathrm{R}_{\text {single }}-$ compare it with Eq. (44) - defined as

$$
\mathcal{E}_{N}^{(\mathrm{s})}(\cdot)=\operatorname{Tr}_{P}\left[\mathcal{E}_{N, g(N)}\left(\cdot \otimes \rho_{P}^{\otimes N g(N)}\right)\right] .
$$

If we now use Eq. (165) together with the monotonicity of the trace distance under partial tracing, we find that

$$
\lim _{N \rightarrow \infty}\left\|\tilde{\mathcal{E}}_{N}^{(\mathrm{s})}\left(\rho^{\otimes N}\right)-\left(\sigma_{g(N)}\right)^{\otimes N}\right\|_{1}=0 .
$$

To conclude the proof, we notice that the sequence of states $\left\{\sigma_{g(N)}\right\}_{N}$ does not need to converge to $\sigma$ with respect to the trace distance. However, if we consider the regularisation of the $E_{\mathcal{F}_{i}}$ 's on these states, we find that

$$
\lim _{N \rightarrow \infty} \frac{1}{N} E_{\mathcal{F}_{i}}\left(\sigma_{g(N)}^{\otimes N}\right)=E_{\mathcal{F}_{i}}(\sigma), \quad i=1,2,
$$

which follows from Eqs. (159) and (164). Then, we can use the asymptotic equivalence of $\mathrm{R}_{\text {multi, }}$ which tells us that there exists a second sequence of allowed operations, and a sub-linear ancilla, such that we can asymptotically transform the state of the system into $\sigma$. This concludes the proof.

The following corollary is stated in Sec. 4.2, and it shows that the bank monotone introduced in Eq. (37) coincides with the relative entropy distance from the set of bank states $\mathcal{F}_{\text {bank }}\left(\bar{E}_{\mathcal{F}_{1}}, \bar{E}_{\mathcal{F}_{2}}\right)$.

Corollary 12. Consider the two-resource theory $R_{\text {multi }}$ with allowed operations $\mathcal{C}_{\text {multi, and invariant }}$ sets $\mathcal{F}_{1}$ and $\mathcal{F}_{2}$ which satisfy the properties F1, F2, F3, and F5b. Suppose the theory satisfies the asymptotic equivalence property with respect to the monotones $E_{\mathcal{F}_{1}}$ and $E_{\mathcal{F}_{2}}$. If the subset of bank states $\mathcal{F}_{\text {bank }}\left(\bar{E}_{\mathcal{F}_{1}}, \bar{E}_{\mathcal{F}_{2}}\right)$ contains a full-rank state, then the bank monotone $f_{\text {bank }}^{\bar{E}_{\mathcal{F}_{1}}, \bar{E}_{\mathcal{F}_{2}}}$ coincides with the relative entropy distance from this subset of states, modulo a multiplicative constant.

Proof. We first notice that Thm. 11 promises us that, under the current assumptions over the theory $\mathrm{R}_{\text {multi, }}$, we can construct a single-resource theory $\mathrm{R}_{\text {single }}$ with allowed operations $\mathcal{C}_{\text {single }}$ as in Def. 10 ,

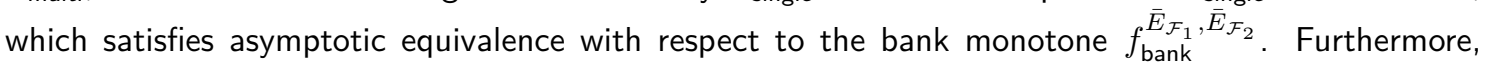
since this monotone satisfies the properties SM1 - SM6 listed in appendix A, we can use Thm. 18 in the same appendix to prove that this single resource theory is reversible. If we then use the results of Ref. [32], we obtain that this monotone is the unique measure of resource for the theory $\mathrm{R}_{\text {single }}$.

What we need to show in this proof is that, actually, both the bank monotone defined in Eq. (37) and the relative entropy distance from the set of bank states $\mathcal{F}_{\text {bank }}\left(\bar{E}_{\mathcal{F}_{1}}, \bar{E}_{\mathcal{F}_{2}}\right)$ satisfy the properties from SM1 to SM6, and therefore by uniqueness these two functions need to coincide (modulo a multiplicative constant). That the bank monotone satisfies these properties is easy to show. Indeed, its monotonicity under the class of operations $\mathcal{C}_{\text {single, }}$, property SM1, is proved in part (a) of Thm. 11. Furthermore, all other properties directly follow from property B1 and the ones listed in Prop. 8.

Showing that the relative entropy distance from the set of states $\mathcal{F}_{\text {bank }}\left(\bar{E}_{\mathcal{F}_{1}}, \bar{E}_{\mathcal{F}_{2}}\right)$ satisfies the same properties is not difficult either. First, we recall that the invariant sets of the theory, $\mathcal{F}_{1}$ and $\mathcal{F}_{2}$, satisfy the properties F1, F2, F3 and F5b by hypothesis. This in turn implies that the subset of bank states under consideration satisfies properties F1, F2 and F5b, as it follows from the Props. 25 and 24 in appendix D.2. That the subset $\mathcal{F}_{\text {bank }}\left(\bar{E}_{\mathcal{F}_{1}}, \bar{E}_{\mathcal{F}_{2}}\right)$ contains a full-rank state, property $\mathrm{F} 3$, is an hypothesis of this corollary.

With the help of the above properties we can show that the relative entropy distance from $\mathcal{F}_{\text {bank }}\left(\bar{E}_{\mathcal{F}_{1}}, \bar{E}_{\mathcal{F}_{2}}\right)$ satisfies the same properties of the bank monotone. That this relative entropy is monotonic under the set of operations $\mathcal{C}_{\text {single, }}$, property SM1, is shown in Prop. 27. Furthermore, property SM2 follows from the definition of relative entropy distance, see Eq. (9), while property SM3 follows from the fact that $\mathcal{F}_{\text {bank }}\left(\bar{E}_{\mathcal{F}_{1}}, \bar{E}_{\mathcal{F}_{2}}\right)$ satisfies the properties F1, F2, and F3. The properties SM4 and SM5 follow from the additivity of the set of bank states, property F5b. Finally, the fact that the monotone scales subextensively, property SM6, is a consequences of the additivity of the set of bank states, as well as of the fact that a full-rank state is contained in this set, properties F5b and F3, respectively. 


\section{D.2 Technical results}

In this section we provide some minor results that are used to prove some of the main theorems in the paper. In particular, the next proposition is used in Sec. 3.3, together with Thm. 5, to show that a multiresource theory satisfying asymptotic equivalence with respect to the relative entropy distances from its invariant sets has unique resource quantifiers. This proposition is already known in the literature, see the references inside the proof.

Proposition 6. Consider a resource theory $R_{\text {multi }}$ with $m$ resources, equipped with the batteries $B_{i}$ 's, where $i=1, \ldots, m$. Suppose the class of allowed operations is $\mathcal{C}_{\text {multi }}$ and the invariant sets are $\left\{\mathcal{F}_{i}\right\}_{i=1}^{m}$. If the invariant set $\mathcal{F}_{i}$ is of the form of Eq. (27), and it satisfies the properties F1 - F5, then the relative entropy distances from this set, $E_{\mathcal{F}_{i}}$, is a regularisable monotone under the class of allowed operations, and it obeys the properties $M 1-M 7$.

Proof. Let us first show that the relative entropy distance $E_{\mathcal{F}_{i}}$ is a monotone for the multi-resource theory $\mathrm{R}_{\text {multi }}$, and that its regularisation is well-defined. These are necessary assumptions we have made in Def. 1. The fact that $E_{\mathcal{F}_{i}}$ is monotonic under the class of allowed operations $\mathcal{C}_{\text {multi }}$, and that in particular it is monotonic under the allowed operations in $\mathcal{C}_{i}$, follows from the argument provided in the last paragraph of Sec. 2.1, and from the fact that $\mathcal{C}_{\text {multi }}$ is obtained from the intersection of all the other classes of allowed operations, see Eq. (11). Furthermore, that the regularisation of $E_{\mathcal{F}_{i}}$ exists follows from the properties F3 and F4. In fact, for all $\rho \in \mathcal{S}(\mathcal{H})$, we have that

$$
\frac{1}{n} E_{\mathcal{F}_{i}}\left(\rho^{\otimes n}\right)=\frac{1}{n} \inf _{\gamma_{n} \in \mathcal{F}(n)} D\left(\rho^{\otimes n} \| \gamma_{n}\right) \leq \frac{1}{n} \inf _{\gamma \in \mathcal{F}} D\left(\rho^{\otimes n} \| \gamma^{\otimes n}\right)=\inf _{\gamma \in \mathcal{F}} D(\rho \| \gamma) \leq D\left(\rho \| \gamma_{\text {full-rank }}\right)
$$

where the first inequality follows from the fact that the invariant sets are closed under tensor product, property $\mathrm{F} 4$, and the second inequality from the fact that they contain at least one full-rank state

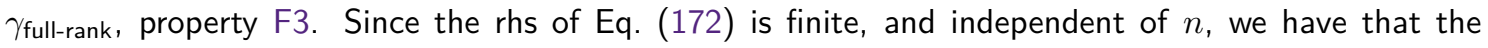
regularisation of the $E_{\mathcal{F}_{i}}$ 's is well-defined.

In order for the monotone to satisfy the property $\mathrm{M} 1$, we can simply choose the states of the battery $B_{i}$ to have a fixed value of the monotones $E_{\mathcal{F}_{j \neq i}}$, for all $j \in\{1, \ldots, m\}$. Property $\mathrm{M} 2$, instead, follows from the fact that we want the batteries to be independent from each other, so as to address them individually. As a result, we choose the global invariant sets to be of the form $\mathcal{F}_{i}=\mathcal{F}_{i, S} \otimes \mathcal{F}_{i, B_{1}} \otimes \ldots \otimes \mathcal{F}_{i, B_{m}}$, where $i=1, \ldots, m$, the main system is $S$, and the $B_{i}$ 's refer to the batteries. This implies that the relative entropy distances from these sets are additive over system and batteries. However, it is still possible for $\mathcal{F}_{i}^{\otimes n}$ to be a proper subset of $\mathcal{F}_{i}^{(n)}$, since on the main systems or batteries we do not ask any additivity property. The validity of property M3 for $E_{\mathcal{F}_{i}}$ follows straightforwardly from the definition of relative entropy distance, see Eq. (9). That $E_{\mathcal{F}_{i}}$ satisfies property M4 follows from property $\mathrm{F} 5$, since for all $\rho_{n} \in \mathcal{S}\left(\mathcal{H}^{\otimes n}\right)$ we have that

$$
\begin{aligned}
E_{\mathcal{F}_{i}}\left(\operatorname{Tr}_{k}\left[\rho_{n}\right]\right) & =\inf _{\gamma_{n-k} \in \mathcal{F}_{i}^{(n-k)}} D\left(\operatorname{Tr}_{k}\left[\rho_{n}\right] \| \gamma_{n-k}\right) \leq \inf _{\gamma_{n} \in \mathcal{F}_{i}^{(n)}} D\left(\operatorname{Tr}_{k}\left[\rho_{n}\right] \| \operatorname{Tr}_{k}\left[\gamma_{n}\right]\right) \\
& \leq \inf _{\gamma_{n} \in \mathcal{F}_{i}^{(n)}} D\left(\rho_{n} \| \gamma_{n}\right)=E_{\mathcal{F}_{i}}\left(\rho_{n}\right),
\end{aligned}
$$

where the first inequality follows from property F5, and the second one from the monotonicity of the relative entropy under CPTP maps. The monotones $E_{\mathcal{F}_{i}}$ 's are also sub-additive, property M5, since for any two states $\rho_{n} \in \mathcal{S}\left(\mathcal{H}^{\otimes n}\right)$ and $\rho_{k} \in \mathcal{S}\left(\mathcal{H}^{\otimes k}\right)$ we have that

$$
\begin{aligned}
E_{\mathcal{F}_{i}}\left(\rho_{n} \otimes \rho_{k}\right) & =\inf _{\gamma_{n+k} \in \mathcal{F}_{i}^{(n+k)}} D\left(\rho_{n} \otimes \rho_{k} \| \gamma_{n+k}\right) \leq \inf _{\gamma_{n} \in \mathcal{F}_{i}^{(n)}, \gamma_{k} \in \mathcal{F}_{i}^{(k)}} D\left(\rho_{n} \otimes \rho_{k} \| \gamma_{n} \otimes \gamma_{k}\right) \\
& =\inf _{\gamma_{n} \in \mathcal{F}_{i}^{(n)}} D\left(\rho_{n} \| \gamma_{n}\right)+\inf _{\gamma_{k} \in \mathcal{F}_{i}^{(k)}} D\left(\rho_{k} \| \gamma_{k}\right)=E_{\mathcal{F}_{i}}\left(\rho_{n}\right)+E_{\mathcal{F}_{i}}\left(\rho_{k}\right),
\end{aligned}
$$

where the inequality follows from property $\mathrm{F} 4$ of the set $\mathcal{F}_{i}$. Property $\mathrm{M} 6$ for the relative entropy distance $E_{\mathcal{F}_{i}}$ follows from similar considerations to the one presented in Eq. (172). In fact, we have that for all $\rho_{n} \in \mathcal{S}\left(\mathcal{H}^{\otimes n}\right)$,

$$
\begin{aligned}
E_{\mathcal{F}_{i}}\left(\rho_{n}\right) & =\inf _{\gamma_{n} \in \mathcal{F}_{i}^{(n)}} D\left(\rho_{n} \| \gamma_{n}\right) \leq D\left(\rho_{n} \| \gamma_{\text {full-rank }}^{\otimes n}\right)=-S\left(\rho_{n}\right)-\operatorname{Tr}\left[\rho_{n} \log \gamma_{\text {full-rank }}^{\otimes n}\right] \\
& \leq-\operatorname{Tr}\left[\rho_{n} \log \gamma_{\text {full-rank }}^{\otimes n}\right] \leq n \log \lambda_{\text {min }}^{-1},
\end{aligned}
$$


where the first inequality follows from the fact that $\mathcal{F}_{i}$ contains a full-rank state, property $\mathrm{F} 3$, the second one from the fact that the von Neumann entropy is non-negative, and the last one from the fact that the optimal case is obtained when $\rho_{n}$ is given by $n$ copies of the pure state associated with the minimum eigenvalue $\lambda_{\text {min }}$ of the full-rank state $\gamma_{\text {full-rank. }}$. Finally, in Ref. [64], Lem. 1, it was shown that the relative entropy distance from a set $\mathcal{F}$ satisfying properties F1, F2, and F3 is asymptotic continuous. In the proof, it was required the set $\mathcal{F}$ to contain the maximally-mixed state. However, as it was noticed in Ref. [63], Lem. C.3, one simply needs $\mathcal{F}$ to contain a full-rank state. Thus, under the above properties on the free set, we have that $E_{\mathcal{F}_{i}}$ satisfies the property M7.

The next proposition collects the properties of the bank monotone defined in Eq. (37).

Proposition 8. Consider a resource theory $R_{\text {multi }}$ with allowed operations $\mathcal{C}_{\text {multi, satisfying asymptotic }}$ equivalence with respect to the monotones $E_{\mathcal{F}_{1}}$ and $E_{\mathcal{F}_{2}}$, i.e. the relative entropy distances from the invariant sets of the theory. Suppose that these sets satisfy the properties F1, F2, F3, and F5b. Then,

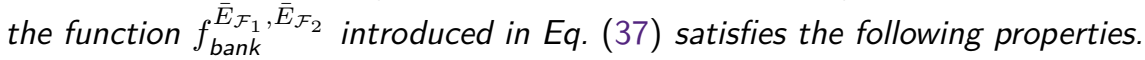

B3 The function $f_{\text {bank }}^{\bar{E}_{\mathcal{F}_{1}}, \bar{E}_{\mathcal{F}_{2}}}$ is additive.

B4 The function $f_{\text {bank }}^{\bar{E}_{\mathcal{F}_{1}}, \bar{E}_{\mathcal{F}_{2}}}$ is monotonic under partial tracing.

B5 The function $f_{\text {bank }}^{\bar{E}_{\mathcal{F}_{1}}, \bar{E}_{\mathcal{F}_{2}}}$ is sub-extensive, i.e., this function scales at most linearly in the number of systems considered. More precisely, for any sequence of states $\left\{\rho_{n} \in \mathcal{S}\left(\mathcal{H}^{\otimes n}\right)\right\}$, we have that $f_{\text {bank }}^{\bar{E}_{\mathcal{F}_{1}}, \bar{E}_{\mathcal{F}_{2}}}\left(\rho_{n}\right)=O(n)$.

B6 The function $f_{\text {bank }}^{\bar{E}_{\mathcal{F}_{1}}, \bar{E}_{\mathcal{F}_{2}}}$ is asymptotic continuous.

B7 The function $f_{\text {bank }}^{\bar{E}_{\mathcal{F}_{1}}, \bar{E}_{\mathcal{F}_{2}}}$ is monotonic under the set of allowed operations $\mathcal{C}_{\text {multi }}$, since $\alpha$ and $\beta$ are non-negative.

Proof. Most of the properties listed above follows straightforwardly from the ones of the invariant sets $\mathcal{F}_{i}$ 's. Here, we only focus on property B4, stating that

$$
f_{\text {bank }}^{\bar{E}_{\mathcal{F}_{1}}, \bar{E}_{\mathcal{F}_{2}}}\left(\operatorname{Tr}_{k}\left[\rho_{n}\right]\right) \leq f_{\text {bank }}^{\bar{E}_{\mathcal{F}_{1}}, \bar{E}_{\mathcal{F}_{2}}}\left(\rho_{n}\right), \quad \forall n, k \in \mathbb{N}, k<n, \forall \rho_{n} \in \mathcal{S}\left(\mathcal{H}^{\otimes n}\right) .
$$

In order to prove the above property, we make use of Lem. 23 and of the definition of bank monotone, see Eq. (37). First, let us divide the $n$ copies of the system into two sets, so that $\mathcal{H}^{\otimes n}=\mathcal{H}^{\otimes k} \otimes \mathcal{H}^{\otimes n-k}$, and in the following equation we refer to $S_{1}$ as the system described by the first $k$ copies, and to $S_{2}$ as the system described by the last $n-k$ copies. In particular, $\rho_{S_{1}}=\operatorname{Tr}_{n-k}\left[\rho_{n}\right] \in \mathcal{S}\left(\mathcal{H}^{\otimes k}\right)$, and $\rho_{S_{2}}=\operatorname{Tr}_{k}\left[\rho_{n}\right] \in \mathcal{S}\left(\mathcal{H}^{\otimes n-k}\right)$. Then, we have the following chain of inequalities

$$
\begin{aligned}
& f_{\text {bank }}^{\bar{E}_{\mathcal{F}_{1}}, \bar{E}_{\mathcal{F}_{2}}}\left(\rho_{n}\right)=\alpha\left(E_{\mathcal{F}_{1}}\left(\rho_{n}\right)-n \bar{E}_{\mathcal{F}_{1}}\right)+\beta\left(E_{\mathcal{F}_{2}}\left(\rho_{n}\right)-n \bar{E}_{\mathcal{F}_{2}}\right) \\
& \geq \alpha\left(E_{\mathcal{F}_{1}}\left(\rho_{S_{1}}\right)+E_{\mathcal{F}_{1}}\left(\rho_{S_{2}}\right)-n \bar{E}_{\mathcal{F}_{1}}\right)+\beta\left(E_{\mathcal{F}_{2}}\left(\rho_{S_{1}}\right)+E_{\mathcal{F}_{2}}\left(\rho_{S_{2}}\right)-n \bar{E}_{\mathcal{F}_{2}}\right) \\
& =\alpha\left(E_{\mathcal{F}_{1}}\left(\rho_{S_{1}}\right)-k \bar{E}_{\mathcal{F}_{1}}\right)+\beta\left(E_{\mathcal{F}_{2}}\left(\rho_{S_{1}}\right)-k \bar{E}_{\mathcal{F}_{2}}\right) \\
& +\alpha\left(E_{\mathcal{F}_{1}}\left(\rho_{S_{2}}\right)-(n-k) \bar{E}_{\mathcal{F}_{1}}\right)+\beta\left(E_{\mathcal{F}_{2}}\left(\rho_{S_{2}}\right)-(n-k) \bar{E}_{\mathcal{F}_{2}}\right) \\
& =f_{\text {bank }}^{\bar{E}_{\mathcal{F}_{1}}, \bar{E}_{\mathcal{F}_{2}}}\left(\rho_{S_{1}}\right)+f_{\text {bank }}^{\bar{E}_{\mathcal{F}_{1}}, \bar{E}_{\mathcal{F}_{2}}}\left(\rho_{S_{2}}\right) \geq f_{\text {bank }}^{\bar{E}_{\mathcal{F}_{1}}, \bar{E}_{\mathcal{F}_{2}}}\left(\rho_{S_{2}}\right)=f_{\text {bank }}^{\bar{E}_{\mathcal{F}_{1}}, \bar{E}_{\mathcal{F}_{2}}}\left(\operatorname{Tr}_{k}\left[\rho_{n}\right]\right) \text {, }
\end{aligned}
$$

where the first inequality follows from Lem. 23, and the second one from the fact that the bank monotone is non-negative, which itself follows from properties B1 and B2.

The following proposition is used in Sec. 3.4 to show that single-resource theories whose class of allowed operations does not increase the average value of a given observable admit a monotone that is asymptotic continuous, see property M7.

Proposition 22. Consider an Hilbert space $\mathcal{H}$ with dimension $d$, an Hermitian operator $A \in \mathcal{B}(\mathcal{H})$, and the function $M_{A}: \mathcal{S}(\mathcal{H}) \rightarrow \mathbb{R}$ defined as

$$
M_{A}(\rho)=\operatorname{Tr}[A \rho]-a_{0}
$$


where $\rho \in \mathcal{S}(\mathcal{H})$ is an element of the state-space, and $a_{0}$ is the minimum eigenvalue of $A$. When $n$ copies of the Hilbert space are considered, $\mathcal{H}_{n}=\otimes_{i=1}^{n} \mathcal{H}^{(i)}$, the above operator is extended as $A_{n}=\sum_{i=1}^{n} A^{(i)}$, where $A^{(i)} \in \mathcal{B}(\mathcal{H})$ acts on the $i$-th copy of the Hilbert space. Then, the function $M_{A}$ is asymptotic continuous.

Proof. Consider two states $\rho_{n}, \sigma_{n} \in \mathcal{S}\left(\mathcal{H}^{\otimes n}\right)$, such that $\left\|\rho_{n}-\sigma_{n}\right\|_{1} \rightarrow 0$ for $n \rightarrow \infty$. We are interested in the difference between the value of the function $M_{A}$ evaluated on $\rho_{n}$ and $\sigma_{n}$. By definition,

$$
\left|M_{A}\left(\rho_{n}\right)-M_{A}\left(\sigma_{n}\right)\right|=\left|\operatorname{Tr}\left[\left(\rho_{n}-\sigma_{n}\right) A_{n}\right]\right| .
$$

Now, we can diagonalise the operator $\rho_{n}-\sigma_{n}=\sum_{\lambda} \lambda\left|\psi_{\lambda}\right\rangle\left\langle\psi_{\lambda}\right|$. Then, we find

$$
\left|\operatorname{Tr}\left[\left(\rho_{n}-\sigma_{n}\right) A_{n}\right]\right|=\left|\sum_{\lambda} \lambda\left\langle\lambda\left|A_{n}\right| \lambda\right\rangle\right| \leq \sum_{\lambda}|\lambda|\left|\left\langle\lambda\left|A_{n}\right| \lambda\right\rangle\right| \leq \sum_{\lambda}|\lambda|\left\|A_{n}\right\|_{\infty},
$$

where we are using the operator norm $\|O\|_{\infty}=\sup _{|\psi\rangle \in \mathcal{H}} \frac{\| O|\psi\rangle \|}{\||\psi\rangle}$, and the last inequality straightforwardly follows from the definition of operator norm. Then, due to the way in which we have defined $A_{n}$, it is easy to show that $\left\|A_{n}\right\|_{\infty}=n\|A\|_{\infty}$, and therefore

$$
\sum_{\lambda}|\lambda|\left\|A_{n}\right\|_{\infty}=n\|A\|_{\infty} \sum_{\lambda}|\lambda|=n\|A\|_{\infty}\left\|\rho_{n}-\sigma_{n}\right\|_{1} .
$$

Finally, notice that $\operatorname{dim} \mathcal{H}_{n}=d^{n}$, where $d$ is fixed by the initial choice of $\mathcal{H}$. Then, we have,

$$
\left|M_{A}\left(\rho_{n}\right)-M_{A}\left(\sigma_{n}\right)\right| \leq n \log d\|A\|_{\infty}\left\|\rho_{n}-\sigma_{n}\right\|_{1} .
$$

If we now divide by $n$ both side of the inequality, we get that

$$
\frac{\left|M_{A}\left(\rho_{n}\right)-M_{A}\left(\sigma_{n}\right)\right|}{n} \leq \log d\|A\|_{\infty}\left\|\rho_{n}-\sigma_{n}\right\|_{1},
$$

and if we send $n \rightarrow \infty$, we obtain that $\frac{1}{n}\left|M_{A}\left(\rho_{n}\right)-M_{A}\left(\sigma_{n}\right)\right| \rightarrow 0$, which proves the theorem.

The following lemma states that, when the sets $\mathcal{F}_{i}$ 's are such that $\mathcal{F}_{i}^{(n)}=\mathcal{F}_{i}^{\otimes n}$ for all $n \in \mathbb{N}$, property F5b, the relative entropy distances from these sets are super-additive. This lemma is used in Prop. 24 and Thm. 11.

Lemma 23. Consider a state $\rho_{S_{1}, S_{2}} \in \mathcal{S}\left(\mathcal{H}^{\otimes 2}\right)$, and suppose that the sets $\mathcal{F}_{1}$ and $\mathcal{F}_{2}$ satisfy property $F 5 b$, that is, $\mathcal{F}_{i}^{(n)}=\mathcal{F}_{i}^{\otimes n}$ for all $n \in \mathbb{N}, i=1,2$. Then, the relative entropy distances from these sets, $E_{\mathcal{F}_{1}}$ and $E_{\mathcal{F}_{2}}$, are such that

$$
E_{\mathcal{F}_{i}}\left(\rho_{S_{1}, S_{2}}\right) \geq E_{\mathcal{F}_{i}}\left(\rho_{S_{1}}\right)+E_{\mathcal{F}_{i}}\left(\rho_{S_{2}}\right), i=1,2,
$$

where $\rho_{S_{1}}=\operatorname{Tr}_{S_{2}}\left[\rho_{S_{1}, S_{2}}\right]$, and similarly $\rho_{S_{2}}=\operatorname{Tr}_{S_{1}}\left[\rho_{S_{1}, S_{2}}\right]$. Furthermore, the above inequality is saturated if and only if $\rho_{S_{1}, S_{2}}=\rho_{S_{1}} \otimes \rho_{S_{2}}$. The result extends trivially to the case in which $n>2$ copies of the system are considered.

Proof. Let us consider the monotone $E_{\mathcal{F}_{1}}$, as the following argument can be equally applied to $E_{\mathcal{F}_{2}}$. By definition of relative entropy distance, we have that

$$
E_{\mathcal{F}_{1}}\left(\rho_{S_{1}, S_{2}}\right)=\inf _{\sigma_{S_{1}, S_{2}} \in \mathcal{F}_{1}^{(2)}} D\left(\rho_{S_{1}, S_{2}} \| \sigma_{S_{1}, S_{2}}\right)=-S\left(\rho_{S_{1}, S_{2}}\right)+\inf _{\sigma_{S_{1}, S_{2}} \in \mathcal{F}_{1}^{(2)}}\left(-\operatorname{Tr}\left[\rho_{S_{1}, S_{2}} \log \sigma_{S_{1}, S_{2}}\right]\right),
$$

where $S\left(\rho_{S_{1}, S_{2}}\right)=-\operatorname{Tr}\left[\rho_{S_{1}, S_{2}} \log \rho_{S_{1}, S_{2}}\right]$ is the Von Neumann entropy of the state $\rho_{S_{1}, S_{2}}$. From the sub-additivity of the Von Neumann entropy, we have that

$$
-S\left(\rho_{S_{1}, S_{2}}\right) \geq-S\left(\rho_{S_{1}}\right)-S\left(\rho_{S_{2}}\right),
$$


while from the property F5b it follows that

$$
\begin{aligned}
\inf _{\sigma_{S_{1}, S_{2}} \in \mathcal{F}_{1}^{(2)}}\left(-\operatorname{Tr}\left[\rho_{S_{1}, S_{2}} \log \sigma_{S_{1}, S_{2}}\right]\right) & =\inf _{\sigma_{S_{1}}, \sigma_{S_{2}} \in \mathcal{F}_{1}}\left(-\operatorname{Tr}\left[\rho_{S_{1}, S_{2}} \log \sigma_{S_{1}} \otimes \sigma_{S_{2}}\right]\right) \\
& =\inf _{\sigma_{S_{1}}, \sigma_{S_{2}} \in \mathcal{F}_{1}}\left(-\operatorname{Tr}\left[\rho_{S_{1}} \log \sigma_{S_{1}}\right]-\operatorname{Tr}\left[\rho_{S_{2}} \log \sigma_{S_{2}}\right]\right) \\
& =\inf _{\sigma_{S_{1}} \in \mathcal{F}_{1}}\left(-\operatorname{Tr}\left[\rho_{S_{1}} \log \sigma_{S_{1}}\right]\right)+\inf _{\sigma_{S_{2}} \in \mathcal{F}_{1}}\left(-\operatorname{Tr}\left[\rho_{S_{2}} \log \sigma_{S_{2}}\right]\right) .
\end{aligned}
$$

From Eqs. (185), (186), and (187) it follows that

$$
\begin{aligned}
E_{\mathcal{F}_{1}}\left(\rho_{S_{1}, S_{2}}\right) & \geq \inf _{\sigma_{S_{1}} \in \mathcal{F}_{1}}\left(-S\left(\rho_{S_{1}}\right)-\operatorname{Tr}\left[\rho_{S_{1}} \log \sigma_{S_{1}}\right]\right)+\inf _{\sigma_{S_{2}} \in \mathcal{F}_{1}}\left(-S\left(\rho_{S_{2}}\right)-\operatorname{Tr}\left[\rho_{S_{2}} \log \sigma_{S_{2}}\right]\right) \\
& =E_{\mathcal{F}_{1}}\left(\rho_{S_{1}}\right)+E_{\mathcal{F}_{1}}\left(\rho_{S_{2}}\right) .
\end{aligned}
$$

The following proposition is used in Sec. 4.1, in Prop. 27, and in Cor. 12. The proposition states that, when the curve of bank states is strictly convex, and we consider $n$ copies of a bank system, the set of bank states $\mathcal{F}_{\text {bank }}^{(n)}$ is given by the tensor product of $n$ copies of states that are in the set $\mathcal{F}_{\text {bank }}$, each of them with the same value of monotones $E_{\mathcal{F}_{1}}$ and $E_{\mathcal{F}_{2}}$.

Proposition 24. Suppose the sets $\mathcal{F}_{1}$ and $\mathcal{F}_{2}$ satisfy property F5b, that is, $\mathcal{F}_{i}^{(n)}=\mathcal{F}_{i}^{\otimes n}$ for all $n \in \mathbb{N}$, $i=1,2$, and the set of bank states $\mathcal{F}_{\text {bank }}$ is represented by a strictly convex curve in the resource diagram. Consider the set of bank states $\mathcal{F}_{\text {bank }}\left(\bar{E}_{\mathcal{F}_{1}}, \bar{E}_{\mathcal{F}_{2}}\right)$ defined in $E q .(35)$, where $E_{\mathcal{F}_{1}}$ and $E_{\mathcal{F}_{2}}$ are the relative entropy distances from the sets $\mathcal{F}_{1}$ and $\mathcal{F}_{2}$, respectively. Then, when $n \in \mathbb{N}$ copies of the bank system are considered, we find that the set of bank states coincides with

$$
\mathcal{F}_{\text {bank }}^{(n)}=\left\{\rho_{1} \otimes \ldots \otimes \rho_{n} \in \mathcal{S}\left(\mathcal{H}^{\otimes n}\right) \mid \exists \bar{E}_{\mathcal{F}_{1}}, \bar{E}_{\mathcal{F}_{2}} \text { such that } \rho_{1}, \ldots, \rho_{n} \in \mathcal{F}_{\text {bank }}\left(\bar{E}_{\mathcal{F}_{1}}, \bar{E}_{\mathcal{F}_{2}}\right)\right\} .
$$

Furthermore, we have that for all subset of bank state $\mathcal{F}_{\text {bank }}\left(\bar{E}_{\mathcal{F}_{1}}, \bar{E}_{\mathcal{F}_{2}}\right) \subset \mathcal{S}(\mathcal{H})$, the corresponding bank subset in $\mathcal{S}\left(\mathcal{H}^{\otimes n}\right)$ is such that

$$
\mathcal{F}_{\text {bank }}^{(n)}\left(\bar{E}_{\mathcal{F}_{1}}, \bar{E}_{\mathcal{F}_{2}}\right)=\mathcal{F}_{\text {bank }}^{\otimes n}\left(\bar{E}_{\mathcal{F}_{1}}, \bar{E}_{\mathcal{F}_{2}}\right) .
$$

Proof. We prove the theorem for $n=2$, as the argument extends trivially for $n>2$. Consider a state $\sigma_{S_{1}, S_{2}} \in \mathcal{S}\left(\mathcal{H}^{\otimes 2}\right)$. From Lem. 23, it follows that

$$
E_{\mathcal{F}_{i}}\left(\sigma_{S_{1}, S_{2}}\right) \geq E_{\mathcal{F}_{i}}\left(\sigma_{S_{1}}\right)+E_{\mathcal{F}_{i}}\left(\sigma_{S_{2}}\right), i=1,2,
$$

where $\sigma_{S_{1}}=\operatorname{Tr}_{S_{2}}\left[\sigma_{S_{1}, S_{2}}\right], \sigma_{S_{2}}=\operatorname{Tr}_{S_{1}}\left[\sigma_{S_{1}, S_{2}}\right]$, and the inequality is saturated iff $\sigma_{S_{1}, S_{2}}=\sigma_{S_{1}} \otimes \sigma_{S_{2}}$. Now, for both the states $\sigma_{S_{1}}, \sigma_{S_{2}} \in \mathcal{S}(\mathcal{H})$, select the bank states $\rho_{P_{1}}, \rho_{P_{2}} \in \mathcal{F}_{\text {bank }}$ such that

$$
E_{\mathcal{F}_{i}}\left(\sigma_{S_{j}}\right) \geq E_{\mathcal{F}_{i}}\left(\rho_{P_{j}}\right), i, j=1,2 .
$$

Recall now that, in the $E_{\mathcal{F}_{1}}-E_{\mathcal{F}_{2}}$ diagram, the curve of bank state is convex (see Prop. 21), and therefore given $\rho_{P_{1}}, \rho_{P_{2}} \in \mathcal{F}_{\text {bank }}$, we can find another $\rho_{P_{3}} \in \mathcal{F}_{\text {bank }}$ such that

$$
\frac{1}{2} E_{\mathcal{F}_{i}}\left(\rho_{P_{1}}\right)+\frac{1}{2} E_{\mathcal{F}_{i}}\left(\rho_{P_{2}}\right) \geq E_{\mathcal{F}_{i}}\left(\rho_{P_{3}}\right), i=1,2,
$$

where the inequality (when the curve is strictly convex) is saturated iff $\rho_{P_{1}}, \rho_{P_{2}}$, and $\rho_{P_{3}}$ all belong to the same subset $\mathcal{F}_{\text {bank }}\left(\bar{E}_{\mathcal{F}_{1}}, \bar{E}_{\mathcal{F}_{2}}\right)$. By combining Eqs. (191), (192), and (193), together with property $\mathrm{F} 5 \mathrm{~b}$ of the sets $\mathcal{F}_{1}$ and $\mathcal{F}_{2}$ (that implies the additivity of the corresponding relative entropy distances), we find that for all $\sigma_{S_{1}, S_{2}} \in \mathcal{S}\left(\mathcal{H}^{\otimes 2}\right)$, it exists a $\rho_{P_{3}} \in \mathcal{F}_{\text {bank }}$ such that

$$
E_{\mathcal{F}_{i}}\left(\sigma_{S_{1}, S_{2}}\right) \geq E_{\mathcal{F}_{i}}\left(\rho_{P_{3}}^{\otimes 2}\right), i=1,2
$$

where the inequality is saturated iff $\sigma_{S_{1}, S_{2}}=\sigma_{S_{1}} \otimes \sigma_{S_{2}}$, and both $\sigma_{S_{1}}$ and $\sigma_{S_{2}}$ belong to the same subset $\mathcal{F}_{\text {bank }}\left(\bar{E}_{\mathcal{F}_{1}}, \bar{E}_{\mathcal{F}_{2}}\right)$. Due to the definition of bank states given in Eq. (34), the thesis of this proposition follows. 
The next proposition shows that, when the invariant sets $\mathcal{F}_{1}$ and $\mathcal{F}_{2}$ are convex sets, the set of bank states $\mathcal{F}_{\text {bank }}\left(\bar{E}_{\mathcal{F}_{1}}, \bar{E}_{\mathcal{F}_{2}}\right)$, defined in Eq. (35), is convex as well. This proposition is used in Sec. 4.1 , as well as in Thm. 12 .

Proposition 25. Suppose that $\mathcal{F}_{1}$ and $\mathcal{F}_{2}$ are convex sets, property $F 2$, and consider the relative entropy distances from these two sets, $E_{\mathcal{F}_{1}}$ and $E_{\mathcal{F}_{2}}$. Then, the set of bank states $\mathcal{F}_{\text {bank }}\left(\bar{E}_{\mathcal{F}_{1}}, \bar{E}_{\mathcal{F}_{2}}\right)$ is convex, as well as its extension to the $n$-copy case, $\mathcal{F}_{\text {bank }}^{(n)}\left(\bar{E}_{\mathcal{F}_{1}}, \bar{E}_{\mathcal{F}_{2}}\right)$, defined in Eq. (190).

Proof. Let us consider two states $\rho_{1}, \rho_{2} \in \mathcal{F}_{\text {bank }}\left(\bar{E}_{\mathcal{F}_{1}}, \bar{E}_{\mathcal{F}_{2}}\right)$. For these two states, there exists $\sigma_{1}, \sigma_{2} \in \mathcal{F}_{1}$ such that

$$
\begin{aligned}
& E_{\mathcal{F}_{1}}\left(\rho_{1}\right)=D\left(\rho_{1} \| \sigma_{1}\right)=\bar{E}_{\mathcal{F}_{1}}, \\
& E_{\mathcal{F}_{1}}\left(\rho_{2}\right)=D\left(\rho_{2} \| \sigma_{2}\right)=\bar{E}_{\mathcal{F}_{1}} .
\end{aligned}
$$

Then, for all $\lambda \in[0,1]$, we have

$$
\begin{aligned}
E_{\mathcal{F}_{1}}\left(\lambda \rho_{1}+(1-\lambda) \rho_{2}\right) & =\inf _{\sigma \in \mathcal{F}_{1}} D\left(\lambda \rho_{1}+(1-\lambda) \rho_{2} \| \sigma\right) \\
& \leq D\left(\lambda \rho_{1}+(1-\lambda) \rho_{2} \| \lambda \sigma_{1}+(1-\lambda) \sigma_{2}\right) \\
& \leq \lambda D\left(\rho_{1} \| \sigma_{1}\right)+(1-\lambda) D\left(\rho_{2} \| \sigma_{2}\right)=\bar{E}_{\mathcal{F}_{1}}
\end{aligned}
$$

where the first inequality follows from the fact that $\mathcal{F}_{1}$ is convex, property $\mathrm{F} 2$, and the second inequality from the joint convexity of the relative entropy. In the same way, it follows that

$$
E_{\mathcal{F}_{2}}\left(\lambda \rho_{1}+(1-\lambda) \rho_{2}\right) \leq \bar{E}_{\mathcal{F}_{2}}
$$

Since $\rho_{1}, \rho_{2} \in \mathcal{F}_{\text {bank }}\left(\bar{E}_{\mathcal{F}_{1}}, \bar{E}_{\mathcal{F}_{2}}\right)$, they satisfy the properties of Eq. (34), and therefore it has to be that, for all $\lambda \in[0,1]$,

$$
E_{\mathcal{F}_{1}}\left(\lambda \rho_{1}+(1-\lambda) \rho_{2}\right)=\bar{E}_{\mathcal{F}_{1}} \text { and } E_{\mathcal{F}_{2}}\left(\lambda \rho_{1}+(1-\lambda) \rho_{2}\right)=\bar{E}_{\mathcal{F}_{2}} .
$$

Thus, we have that $\lambda \rho_{1}+(1-\lambda) \rho_{2} \in \mathcal{F}_{\text {bank }}\left(\bar{E}_{\mathcal{F}_{1}}, \bar{E}_{\mathcal{F}_{2}}\right)$. This result can be extended to the case of $n \in \mathbb{N}$ copies of the system, where the bank set $\mathcal{F}_{\text {bank }}^{(n)}\left(\bar{E}_{\mathcal{F}_{1}}, \bar{E}_{\mathcal{F}_{2}}\right)$ is defined as in Eq. (190). In this case, the proof is analogous to the one considered above, with the exception that in the rhs of Eqs. (195), and of the following ones, we add the multiplicative factor $n$.

The next lemma is used in Prop. 27. The lemma states that, given the class of operations $\mathcal{C}_{\text {multi }}$ for which $\mathcal{F}_{1}$ and $\mathcal{F}_{2}$ are invariant sets, the set of bank states $\mathcal{F}_{\text {bank, }}$, defined in Eq. (34), is invariant as well.

Lemma 26. Consider a resource theory $R_{\text {multi }}$ with allowed operations $\mathcal{C}_{\text {multi, }}$ and two invariant sets $\mathcal{F}_{1}$ and $\mathcal{F}_{2}$. Consider the subset of bank states $\mathcal{F}_{\text {bank }}\left(\bar{E}_{\mathcal{F}_{1}}, \bar{E}_{\mathcal{F}_{2}}\right)$ as defined in Eq. (35). Then, for all $\mathcal{E} \in \mathcal{C}_{\text {multi, }}$, we have that $\mathcal{F}_{\text {bank }}\left(\bar{E}_{\mathcal{F}_{1}}, \bar{E}_{\mathcal{F}_{2}}\right)$ is an invariant set, that is

$$
\mathcal{E}(\rho) \in \mathcal{F}_{\text {bank }}\left(\bar{E}_{\mathcal{F}_{1}}, \bar{E}_{\mathcal{F}_{2}}\right), \quad \forall \rho \in \mathcal{F}_{\text {bank }}\left(\bar{E}_{\mathcal{F}_{1}}, \bar{E}_{\mathcal{F}_{2}}\right)
$$

Analogously, the set of bank states describing $n$ copies of the bank system is invariant under the class of allowed operations $\mathcal{C}_{\text {multi }}^{(n)}$.

Proof. Let us consider $\rho \in \mathcal{F}_{\text {bank }}\left(\bar{E}_{\mathcal{F}_{1}}, \bar{E}_{\mathcal{F}_{2}}\right)$, as well as the state $\mathcal{E}(\rho)$ obtained by applying the map $\mathcal{E} \in \mathcal{C}_{\text {multi }}$ to the bank state. Due to the monotonicity of $E_{\mathcal{F}_{1}}$ and $E_{\mathcal{F}_{2}}$, we have that $E_{\mathcal{F}_{1}}(\mathcal{E}(\rho)) \leq$ $E_{\mathcal{F}_{1}}(\rho)$, and $E_{\mathcal{F}_{2}}(\mathcal{E}(\rho)) \leq E_{\mathcal{F}_{2}}(\rho)$. Recall now that $\rho$ is a bank state, which implies that $\forall \sigma \in \mathcal{S}(\mathcal{H})$, one (or more) of the following options holds

1. $E_{\mathcal{F}_{1}}(\sigma)>E_{\mathcal{F}_{1}}(\rho)$.

2. $E_{\mathcal{F}_{2}}(\sigma)>E_{\mathcal{F}_{2}}(\rho)$.

3. $E_{\mathcal{F}_{1}}(\sigma)=E_{\mathcal{F}_{1}}(\rho)$ and $E_{\mathcal{F}_{2}}(\sigma)=E_{\mathcal{F}_{2}}(\rho)$. 
However, the monotonicity conditions given by $E_{\mathcal{F}_{1}}$ and $E_{\mathcal{F}_{2}}$ implies that $\mathcal{E}(\rho)$ violates options 1 and 2 , so that option 3 is the only possible one. But this implies that $E_{\mathcal{F}_{1}}(\mathcal{E}(\rho))=E_{\mathcal{F}_{1}}(\rho)$ and $E_{\mathcal{F}_{2}}(\mathcal{E}(\rho))=E_{\mathcal{F}_{2}}(\rho)$, meaning that $\mathcal{E}(\rho) \in \mathcal{F}_{\text {bank }}\left(\bar{E}_{\mathcal{F}_{1}}, \bar{E}_{\mathcal{F}_{2}}\right)$. The same argument applies to the set $\mathcal{F}_{\text {bank }}^{(n)}$, when $n$ copies of the system are considered. Indeed, this case is analogous to the one considered above, with the sole difference that now the state $\rho \in \mathcal{F}_{\text {bank }}^{(n)}$, the state $\sigma \in \mathcal{S}\left(\mathcal{H}^{\otimes n}\right)$, and the operations we use are in the class $\mathcal{C}_{\text {multi }}^{(n)}$ defined in Sec. 2.2.

The last proposition of this section shows that the relative entropy distance from the set $\mathcal{F}_{\text {bank }}\left(\bar{E}_{\mathcal{F}_{1}}, \bar{E}_{\mathcal{F}_{2}}\right)$ is monotonic under the class of operations $\mathcal{C}_{\text {single }}$, introduced in Def. 10 . This proposition is used in Cor. 12.

Proposition 27. Consider a multi-resource theory $R_{\text {multi }}$ with two resources, whose allowed operations $\mathcal{C}_{\text {multi }}$ leave the sets $\mathcal{F}_{1}$ and $\mathcal{F}_{2}$ invariant. Suppose these invariant sets satisfy the properties F1, F2, F3, and F5b. Then, the relative entropy distance from the subset of bank states $\mathcal{F}_{\text {bank }}\left(\bar{E}_{\mathcal{F}_{1}}, \bar{E}_{\mathcal{F}_{2}}\right)$ is monotonic under both the class of operations $\mathcal{C}_{\text {multi }}$ and the class $\mathcal{C}_{\text {single }}$ introduced in Def. 10.

Proof. 1. Here we show monotonicity of the relative entropy distance with respect to the addition of an ancillary system described by $n \in \mathbb{N}$ copies of a bank states. Consider the state $\rho \in \mathcal{S}(\mathcal{H})$, and the bank state $\rho_{P} \in \mathcal{F}_{\text {bank }}\left(\bar{E}_{\mathcal{F}_{1}}, \bar{E}_{\mathcal{F}_{2}}\right)$. Then, we have

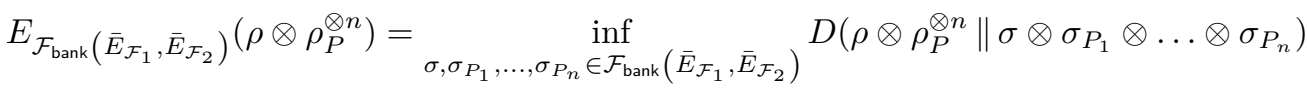

$$
\begin{aligned}
& =\inf _{\sigma \in \mathcal{F}_{\text {bank }}\left(\bar{E}_{\mathcal{F}_{1}}, \bar{E}_{\mathcal{F}_{2}}\right)} D(\rho \| \sigma)+\sum_{i=1}^{n} \inf _{\sigma_{P_{i}} \in \mathcal{F}_{\text {bank }}\left(\bar{E}_{\mathcal{F}_{1}}, \bar{E}_{\mathcal{F}_{2}}\right)} D\left(\rho_{P} \| \sigma_{P_{i}}\right) \\
& =\inf _{\sigma \in \mathcal{F}_{\text {bank }}\left(\bar{E}_{\mathcal{F}_{1}}, \bar{E}_{\mathcal{F}_{2}}\right)} D(\rho \| \sigma)=E_{\mathcal{F}_{\text {bank }}\left(\bar{E}_{\mathcal{F}_{1}}, \bar{E}_{\mathcal{F}_{2}}\right)}(\rho)
\end{aligned}
$$

where the first equality follows from Prop. 24 , and the last one from the fact that $\rho_{P} \in \mathcal{F}_{\text {bank }}\left(\bar{E}_{\mathcal{F}_{1}}, \bar{E}_{\mathcal{F}_{2}}\right)$.

2. Now we show monotonicity of the relative entropy distance with respect to the allowed operations $\mathcal{C}_{\text {mulit }}$. Let us consider a state $\rho \in \mathcal{S}(\mathcal{H})$, together with an operation $\mathcal{E} \in \mathcal{C}_{\text {multi. }}$ Then, we have that

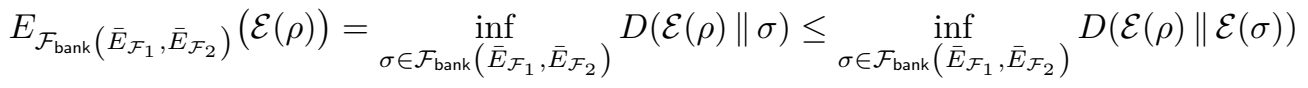

$$
\begin{aligned}
& \leq \inf _{\sigma \in \mathcal{F}_{\text {bank }}\left(\bar{E}_{\mathcal{F}_{1}}, \bar{E}_{\mathcal{F}_{2}}\right)} D(\rho \| \sigma)=E_{\mathcal{F}_{\text {bank }}\left(\bar{E}_{\mathcal{F}_{1}}, \bar{E}_{\mathcal{F}_{2}}\right)}(\rho)
\end{aligned}
$$

where the first inequality follows from Lem. 26, and the second one from the monotonicity of the relative entropy under CPTP maps. This result trivially extends to the case in which we have multiple copies of the system, since in Lem. 26 we have shown that $\mathcal{F}_{\text {bank }}^{(n)}$ is invariant under the allowed operations $\mathcal{C}_{\text {multi }}^{(n)}$ for all $n \in \mathbb{N}$.

3. We show the monotonicity of the relative entropy with respect to partial tracing when the ancillary system is composed by just one copy. However, the result straightforwardly extends to the case in which the ancillary system is composed by $n \in \mathbb{N}$ copies. Let us consider the state $\rho_{S_{1}, S_{2}} \in \mathcal{S}\left(\mathcal{H}^{\otimes 2}\right)$. Then, we have that

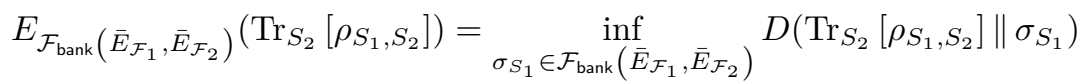

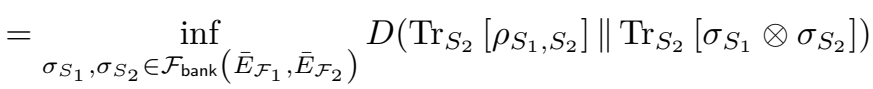

$$
\begin{aligned}
& \leq \inf _{\sigma_{S_{1}}, \sigma_{S_{2}} \in \mathcal{F}_{\text {bank }}\left(\bar{E}_{\mathcal{F}_{1}}, \bar{E}_{\mathcal{F}_{2}}\right)} D\left(\rho_{S_{1}, S_{2}} \| \sigma_{S_{1}} \otimes \sigma_{S_{2}}\right) \\
& =E_{\mathcal{F}_{\text {bank }}\left(\bar{E}_{\mathcal{F}_{1}}, \bar{E}_{\mathcal{F}_{2}}\right)}\left(\rho_{S_{1}, S_{2}}\right) \text {, }
\end{aligned}
$$

where the second equality follows from Prop. 24, while the inequality follows from the monotonicity of the relative entropy distance under CPTP maps. 


\section{References}

[1] Charles H. Bennett, David P. DiVincenzo, John A. Smolin, and William K. Wootters. Mixedstate entanglement and quantum error correction. Physical Review A, 54(5):3824, 1996. doi:10.1103/PhysRevA.54.3824.

[2] Eric M. Rains. Entanglement purification via separable superoperators. arXiv:quant-ph/9707002, 1997. URL https://arxiv.org/abs/quant-ph/9707002.

[3] Vlatko Vedral and Martin B. Plenio. Entanglement measures and purification procedures. Physical Review A, 57(3):1619-1633, 1998. doi:10.1103/PhysRevA.57.1619.

[4] Eric M. Rains. Bound on distillable entanglement. Physical Review A, 60(1):179-184, 1999. doi:10.1103/PhysRevA.60.179.

[5] Ryszard Horodecki, Paweł Horodecki, Michał Horodecki, and Karol Horodecki. Quantum entanglement. Reviews of Modern Physics, 81(2):865-942, 2009. doi:10.1103/RevModPhys.81.865.

[6] Dominik Janzing, Pawel Wocjan, Robert Zeier, Rubino Geiss, and Thomas Beth. Thermodynamic Cost of Reliability and Low Temperatures: Tightening Landauer's Principle and the Second Law. International Journal of Theoretical Physics, 39(12):2717-2753, 2000. doi:10.1023/A:1026422630734.

[7] Michał Horodecki, Paweł Horodecki, and Jonathan Oppenheim. Reversible transformations from pure to mixed states and the unique measure of information. Physical Review A, 67(6):062104, 2003. doi:10.1103/PhysRevA.67.062104.

[8] Lídia del Rio, Johan Åberg, Renato Renner, Oscar Dahlsten, and Vlatko Vedral. The thermodynamic meaning of negative entropy. Nature, 474(7349):61-63, 2011. doi:10.1038/nature10123.

[9] Oscar C. O. Dahlsten, Renato Renner, Elisabeth Rieper, and Vlatko Vedral. Inadequacy of von Neumann entropy for characterizing extractable work. New Journal of Physics, 13(053015):053015, 2011. doi:10.1088/1367-2630/13/5/053015.

[10] Fernando G. S. L. Brandão, Michał Horodecki, Jonathan Oppenheim, Joseph M. Renes, and Robert W. Spekkens. Resource Theory of Quantum States Out of Thermal Equilibrium. Physical Review Letters, 111(25):250404, 2013. doi:10.1103/PhysRevLett.111.250404.

[11] Michał Horodecki and Jonathan Oppenheim. Fundamental limitations for quantum and nanoscale thermodynamics. Nature Communications, 4:2059, 2013. doi:10.1038/ncomms3059.

[12] Paul Skrzypczyk, Anthony J. Short, and Sandu Popescu. Work extraction and thermodynamics for individual quantum systems. Nature Communications, 5:4185, 2014. doi:10.1038/ncomms5185.

[13] Rodrigo Gallego, Jens Eisert, and Henrik Wilming. Thermodynamic work from operational principles. New Journal of Physics, 18(10):103017, 2016. doi:10.1088/1367-2630/18/10/103017.

[14] Gilad Gour and Robert W Spekkens. The resource theory of quantum reference frames: manipulations and monotones. New Journal of Physics, 10(3):033023, 2008. doi:10.1088/13672630/10/3/033023.

[15] Gilad Gour, Iman Marvian, and Robert W. Spekkens. Measuring the quality of a quantum reference frame: The relative entropy of frameness. Physical Review A, 80(1):012307, 2009. doi:10.1103/PhysRevA.80.012307.

[16] Iman Marvian and Robert W. Spekkens. The theory of manipulations of pure state asymmetry: I. Basic tools, equivalence classes and single copy transformations. New Journal of Physics, 15(3): 033001, 2013. doi:10.1088/1367-2630/15/3/033001.

[17] Andrea Mari and Jens Eisert. Positive Wigner Functions Render Classical Simulation of Quantum Computation Efficient. Physical Review Letters, 109(23):230503, 2012. doi:10.1103/PhysRevLett.109.230503.

[18] Victor Veitch, S. A. Hamed Mousavian, Daniel Gottesman, and Joseph Emerson. The resource theory of stabilizer quantum computation. New Journal of Physics, 16(1):013009, 2014. doi:10.1088/1367-2630/16/1/013009.

[19] Victor Veitch, Christopher Ferrie, David Gross, and Joseph Emerson. Negative quasi-probability as a resource for quantum computation. New Journal of Physics, 14(11):113011, 2012. doi:10.1088/1367-2630/14/11/113011.

[20] Elliott H. Lieb and Jakob Yngvason. The physics and mathematics of the second law of thermodynamics. Physics Reports, 310(1):1-96, 1999. doi:10.1016/S0370-1573(98)00082-9.

[21] Elliott H. Lieb and Jakob Yngvason. The entropy concept for non-equilibrium states. Proc. R. Soc. $A, 469(2158): 20130408,2013$. doi:10.1098/rspa.2013.0408. 
[22] Mirjam Weilenmann, Lea Kraemer, Philippe Faist, and Renato Renner. Axiomatic Relation between Thermodynamic and Information-Theoretic Entropies. Physical Review Letters, 117(26):260601, 2016. doi:10.1103/PhysRevLett.117.260601.

[23] Tobias Fritz. Resource convertibility and ordered commutative monoids. Mathematical Structures in Computer Science, 27:850-918, 2017. doi:10.1017/S0960129515000444.

[24] Lídia del Rio, Lea Kraemer, and Renato Renner. Resource theories of knowledge. arXiv:1511.08818 [cond-mat, physics:math-ph, physics:quant-ph], 2015. URL http://arxiv.org/abs/1511. 08818.

[25] Bob Coecke, Tobias Fritz, and Robert W. Spekkens. A mathematical theory of resources. Information and Computation, 250:59-86, 2016. doi:10.1016/j.ic.2016.02.008.

[26] Anurag Anshu, Min-Hsiu Hsieh, and Rahul Jain. Quantifying Resources in General Resource Theory with Catalysts. Physical Review Letters, 121(19):190504, 2018. doi:10.1103/PhysRevLett.121.190504.

[27] Fernando Brando, Michał Horodecki, Nelly Ng, Jonathan Oppenheim, and Stephanie Wehner. The second laws of quantum thermodynamics. Proceedings of the National Academy of Sciences, 112 (11):3275-3279, 2015. doi:10.1073/pnas.1411728112.

[28] Sandu Popescu and Daniel Rohrlich. Thermodynamics and the measure of entanglement. Physical Review A, 56(5):R3319-R3321, 1997. doi:10.1103/PhysRevA.56.R3319.

[29] Michat Horodecki, Jonathan Oppenheim, and Ryszard Horodecki. Are the Laws of Entanglement Theory Thermodynamical? Physical Review Letters, 89(24):240403, 2002. doi:10.1103/PhysRevLett.89.240403.

[30] Fernando G. S. L. Brandão and Martin B. Plenio. Entanglement theory and the second law of thermodynamics. Nature Physics, 4(11):873-877, 2008. doi:10.1038/nphys1100.

[31] Fernando G. S. L. Brandão and Martin B. Plenio. A Reversible Theory of Entanglement and its Relation to the Second Law. Communications in Mathematical Physics, 295(3):829-851, 2010. doi:10.1007/s00220-010-1003-1.

[32] Michat Horodecki and Jonathan Oppenheim. (quantumness in the context of) resource theories. International Journal of Modern Physics B, 27(01n03):1345019, 2012. doi:10.1142/S0217979213450197.

[33] Fernando G. S. L. Brandão and Gilad Gour. Reversible Framework for Quantum Resource Theories. Physical Review Letters, 115(7):070503, 2015. doi:10.1103/PhysRevLett.115.070503.

[34] Lluís Masanes and Jonathan Oppenheim. A general derivation and quantification of the third law of thermodynamics. Nature Communications, 8:14538, 2017. doi:10.1038/ncomms14538.

[35] Carlo Sparaciari, Jonathan Oppenheim, and Tobias Fritz. Resource theory for work and heat. Physical Review A, 96(5):052112, 2017. doi:10.1103/PhysRevA.96.052112.

[36] Manabendra N. Bera, Arnau Riera, Maciej Lewenstein, and Andreas Winter. Thermodynamics as a Consequence of Information Conservation. Quantum, 3:121, 2019. doi:10.22331/q-2019-02-14121.

[37] Gilad Gour, Markus P. Müller, Varun Narasimhachar, Robert W. Spekkens, and Nicole Yunger Halpern. The resource theory of informational nonequilibrium in thermodynamics. Physics Reports, 583:1-58, 2015. doi:10.1016/j.physrep.2015.04.003.

[38] Johan Åberg. Quantifying Superposition. arXiv:quant-ph/0612146, 2006. URL http://arxiv. org/abs/quant-ph/0612146.

[39] Tillmann Baumgratz, Marcus Cramer, and Martin B. Plenio. Quantifying Coherence. Physical Review Letters, 113(14):140401, 2014. doi:10.1103/PhysRevLett.113.140401.

[40] Andreas Winter and Dong Yang. Operational Resource Theory of Coherence. Physical Review Letters, 116(12):120404, 2016. doi:10.1103/PhysRevLett.116.120404.

[41] David Slepian and Jack K. Wolf. Noiseless coding of correlated information sources. IEEE Transactions on Information Theory, 19(4):471-480, 1973. doi:10.1109/TIT.1973.1055037.

[42] Michał Horodecki, Jonathan Oppenheim, and Andreas Winter. Partial quantum information. Nature, 436(7051):673-676, 2005. doi:10.1038/nature03909.

[43] Mehdi Ahmadi, David Jennings, and Terry Rudolph. The WignerArakiYanase theorem and the quantum resource theory of asymmetry. New Journal of Physics, 15(1):013057, 2013. doi:10.1088/1367-2630/15/1/013057. 
[44] Uttam Singh, Manabendra N. Bera, Himadri S. Dhar, and Arun K. Pati. Maximally coherent mixed states: Complementarity between maximal coherence and mixedness. Physical Review A, 91(5):052115, 2015. doi:10.1103/PhysRevA.91.052115.

[45] Alexander Streltsov, Eric Chitambar, Swapan Rana, Manabendra N. Bera, Andreas Winter, and Maciej Lewenstein. Entanglement and Coherence in Quantum State Merging. Physical Review Letters, 116(24):240405, 2016. doi:10.1103/PhysRevLett.116.240405.

[46] Eric Chitambar and Min-Hsiu Hsieh. Relating the Resource Theories of Entanglement and Quantum Coherence. Physical Review Letters, 117(2):020402, 2016. doi:10.1103/PhysRevLett.117.020402.

[47] Paul Erker, Mark T. Mitchison, Ralph Silva, Mischa P. Woods, Nicolas Brunner, and Marcus Huber. Autonomous Quantum Clocks: Does Thermodynamics Limit Our Ability to Measure Time? Physical Review X, 7(3):031022, 2017. doi:10.1103/PhysRevX.7.031022.

[48] Yelena Guryanova, Sandu Popescu, Anthony J. Short, Ralph Silva, and Paul Skrzypczyk. Thermodynamics of quantum systems with multiple conserved quantities. Nature Communications, 7 : 12049, 2016. doi:10.1038/ncomms12049.

[49] Nicole Yunger Halpern, Philippe Faist, Jonathan Oppenheim, and Andreas Winter. Microcanonical and resource-theoretic derivations of the thermal state of a quantum system with noncommuting charges. Nature Communications, 7:12051, 2016. doi:10.1038/ncomms12051.

[50] Nicole Yunger Halpern and Joseph M. Renes. Beyond heat baths: Generalized resource theories for small-scale thermodynamics. Physical Review E, 93(2):022126, 2016. doi:10.1103/PhysRevE.93.022126.

[51] Matteo Lostaglio, David Jennings, and Terry Rudolph. Thermodynamic resource theories, noncommutativity and maximum entropy principles. New Journal of Physics, 19(4):043008, 2017. doi:10.1088/1367-2630/aa617f.

[52] Nicole Yunger Halpern. Beyond heat baths II: framework for generalized thermodynamic resource theories. Journal of Physics A: Mathematical and Theoretical, 51(9):094001, 2018. doi:10.1088/1751-8121/aaa62f.

[53] Sandu Popescu, Ana Belén Sainz, Anthony J. Short, and Andreas Winter. Quantum reference frames and their applications to thermodynamics. Philosophical Transactions of the Royal Society A: Mathematical, Physical and Engineering Sciences, 376(2123):20180111, 2018. doi:10.1098/rsta.2018.0111.

[54] Eric Chitambar and Gilad Gour. Quantum resource theories. Reviews of Modern Physics, 91(2): 025001, 2019. doi:10.1103/RevModPhys.91.025001.

[55] Joseph M. Renes. Work cost of thermal operations in quantum thermodynamics. The European Physical Journal Plus, 129(7):153, 2014. doi:10.1140/epjp/i2014-14153-8.

[56] Charles H. Bennett, David P. DiVincenzo, Christopher A. Fuchs, Tal Mor, Eric Rains, Peter W. Shor, John A. Smolin, and William K. Wootters. Quantum nonlocality without entanglement. Physical Review A, 59(2):1070, 1999. doi:10.1103/PhysRevA.59.1070.

[57] Göran Lindblad. Completely positive maps and entropy inequalities. Communications in Mathematical Physics, 40(2):147-151, 1975. doi:10.1007/BF01609396.

[58] Charles H. Bennett, David P. DiVincenzo, Tal Mor, Peter W. Shor, John A. Smolin, and Barbara M. Terhal. Unextendible Product Bases and Bound Entanglement. Physical Review Letters, 82(26): 5385-5388, 1999. doi:10.1103/PhysRevLett.82.5385.

[59] Lidia del Rio, Philippe Faist, Jonathan Oppenheim, Carlo Maria Scandolo, and Carlo Sparaciari. In preparation.

[60] Lea Kraemer and Lidia del Rio. Currencies in resource theories. arXiv:1605.01064 [cond-mat, physics:math-ph, physics:quant-ph], 2016. URL https://arxiv.org/abs/1605.01064.

[61] K. G. H. Vollbrecht and R. F. Werner. Entanglement measures under symmetry. Physical Review A, 64(6):062307, 2001. doi:10.1103/PhysRevA.64.062307.

[62] Michał Horodecki. Entanglement Measures. Quantum Information and Computation, 1(1):3 - 26, 2001. doi:10.26421/QIC1.1.

[63] Fernando G. S. L. Brandão and Martin B. Plenio. A Generalization of Quantum Steins Lemma. Communications in Mathematical Physics, 295(3):791-828, 2010. doi:10.1007/s00220-010-1005z.

[64] Barbara Synak-Radtke and Michał Horodecki. On asymptotic continuity of functions of quantum states. Journal of Physics A: Mathematical and General, 39(26):L423, 2006. doi:10.1088/03054470/39/26/L02. 
[65] Rolf Landauer. Irreversibility and heat generation in the computing process. IBM journal of research and development, 5(3):183-191, 1961. doi:10.1147/rd.53.0183.

[66] Charles H. Bennett. The thermodynamics of computationa review. International Journal of Theoretical Physics, 21(12):905-940, 1982. doi:10.1007/BF02084158.

[67] Edwin T. Jaynes. Information theory and statistical mechanics. Physical review, 106(4):620, 1957. doi:10.1103/PhysRev.106.620.

[68] Renato Renner. Symmetry of large physical systems implies independence of subsystems. Nature Physics, 3(9):645-649, 2007. doi:10.1038/nphys684.

[69] N H Y Ng, L Mančinska, C Cirstoiu, J Eisert, and S Wehner. Limits to catalysis in quantum thermodynamics. New Journal of Physics, 17(8):085004, 2015. doi:10.1088/1367-2630/17/8/085004.

[70] Wim van Dam and Patrick Hayden. Universal entanglement transformations without communication. Physical Review A, 67(6):060302, 2003. doi:10.1103/PhysRevA.67.060302.

[71] Karen V. Hovhannisyan, Martí Perarnau-Llobet, Marcus Huber, and Antonio Acín. Entanglement Generation is Not Necessary for Optimal Work Extraction. Physical Review Letters, 111(24): 240401, 2013. doi:10.1103/PhysRevLett.111.240401.

[72] Marcus Huber, Martí Perarnau-Llobet, Karen V. Hovhannisyan, Paul Skrzypczyk, Claude Klöckl, Nicolas Brunner, and Antonio Acín. Thermodynamic cost of creating correlations. New Journal of Physics, 17(6):065008, 2015. doi:10.1088/1367-2630/17/6/065008.

[73] H. Wilming, R. Gallego, and J. Eisert. Second law of thermodynamics under control restrictions. Physical Review E, 93(4):042126, 2016. doi:10.1103/PhysRevE.93.042126.

[74] Cédric Bény, Christopher T. Chubb, Terry Farrelly, and Tobias J. Osborne. Energy cost of entanglement extraction in complex quantum systems. Nature Communications, 9(1):3792, 2018. doi:10.1038/s41467-018-06153-w.

[75] J. Lekscha, H. Wilming, J. Eisert, and R. Gallego. Quantum thermodynamics with local control. Physical Review E, 97(2):022142, 2018. doi:10.1103/PhysRevE.97.022142.

[76] Vlatko Vedral, Martin B. Plenio, M. A. Rippin, and Peter L. Knight. Quantifying Entanglement. Physical Review Letters, 78(12):2275-2279, 1997. doi:10.1103/PhysRevLett.78.2275.

[77] Koenraad Audenaert, Jens Eisert, E. Jané, Martin B. Plenio, Shashank Virmani, and Bart De Moor. Asymptotic Relative Entropy of Entanglement. Physical Review Letters, 87(21):217902, 2001. doi:10.1103/PhysRevLett.87.217902.

[78] Adam Miranowicz and Satoshi Ishizaka. Closed formula for the relative entropy of entanglement. Physical Review A, 78(3):032310, 2008. doi:10.1103/PhysRevA.78.032310.

[79] Alvaro M. Alhambra, Lluis Masanes, Jonathan Oppenheim, and Christopher Perry. Entanglement fluctuation theorems. Physical Review A, 100:012317, 2019. doi:10.1103/PhysRevA.100.012317.

[80] Alvaro M. Alhambra, Lluis Masanes, Jonathan Oppenheim, and Christopher Perry. Fluctuating Work: From Quantum Thermodynamical Identities to a Second Law Equality. Physical Review X, 6(4):041017, 2016. doi:10.1103/PhysRevX.6.041017.

[81] Joseph M. Renes. Relative submajorization and its use in quantum resource theories. Journal of Mathematical Physics, 57(12):122202, 2016. doi:10.1063/1.4972295.

[82] Benjamin Morris and Gerardo Adesso. Quantum coherence fluctuation relations. Journal of Physics A: Mathematical and Theoretical, 51(41):414007, 2018. doi:10.1088/1751-8121/aac115.

[83] Aram W. Harrow. Entanglement spread and clean resource inequalities. In XVIth International Congress on Mathematical Physics, pages 536-540. World Scientific, 2010. doi:10.1142/9789814304634_0046.

[84] Robert Alicki and Mark Fannes. Entanglement boost for extractable work from ensembles of quantum batteries. Physical Review E, 87(4), 2013. doi:10.1103/PhysRevE.87.042123.

[85] Johan Åberg. Catalytic Coherence. Physical Review Letters, 113(15):150402, 2014. doi:10.1103/PhysRevLett.113.150402.

[86] Matteo Lostaglio, David Jennings, and Terry Rudolph. Description of quantum coherence in thermodynamic processes requires constraints beyond free energy. Nature Communications, 6: 6383, 2015. doi:10.1038/ncomms7383.

[87] Hyukjoon Kwon, Hyunseok Jeong, David Jennings, Benjamin Yadin, and M. S. Kim. Clock-Work Trade-Off Relation for Coherence in Quantum Thermodynamics. Physical Review Letters, 120 (15):150602, 2018. doi:10.1103/PhysRevLett.120.150602.

[88] Matthew J. Donald, Michał Horodecki, and Oliver Rudolph. The uniqueness theorem for entanglement measures. Journal of Mathematical Physics, 43(9):4252-4272, 2002. doi:10.1063/1.1495917. 
[89] Matthias Christandl. The Structure of Bipartite Quantum States - Insights from Group Theory and Cryptography. arXiv:quant-ph/0604183, 2006. URL https://arxiv.org/abs/quant-ph/ 0604183.

[90] Benjamin Schumacher. Quantum coding. Physical Review A, 51(4):2738-2747, 1995. doi:10.1103/PhysRevA.51.2738. 